\title{
Redox Mechanisms in Neurodegeneration: From Disease Outcomes to Therapeutic Opportunities
}

\author{
Juan I. Sbodio, Solomon H. Snyder, ${ }^{1-3}$ and Bindu D. Paul ${ }^{1}$
}

\begin{abstract}
Significance: Once considered to be mere by-products of metabolism, reactive oxygen, nitrogen and sulfur species are now recognized to play important roles in diverse cellular processes such as response to pathogens and regulation of cellular differentiation. It is becoming increasingly evident that redox imbalance can impact several signaling pathways. For instance, disturbances of redox regulation in the brain mediate neurodegeneration and alter normal cytoprotective responses to stress. Very often small disturbances in redox signaling processes, which are reversible, precede damage in neurodegeneration.

Recent Advances: The identification of redox-regulated processes, such as regulation of biochemical pathways involved in the maintenance of redox homeostasis in the brain has provided deeper insights into mechanisms of neuroprotection and neurodegeneration. Recent studies have also identified several post-translational modifications involving reactive cysteine residues, such as nitrosylation and sulfhydration, which fine-tune redox regulation. Thus, the study of mechanisms via which cell death occurs in several neurodegenerative disorders, reveal several similarities and dissimilarities. Here, we review redox regulated events that are disrupted in neurodegenerative disorders and whose modulation affords therapeutic opportunities.

Critical Issues: Although accumulating evidence suggests that redox imbalance plays a significant role in progression of several neurodegenerative diseases, precise understanding of redox regulated events is lacking. Probes and methodologies that can precisely detect and quantify in vivo levels of reactive oxygen, nitrogen and sulfur species are not available.

Future Directions: Due to the importance of redox control in physiologic processes, organisms have evolved multiple pathways to counteract redox imbalance and maintain homeostasis. Cells and tissues address stress by harnessing an array of both endogenous and exogenous redox active substances. Targeting these pathways can help mitigate symptoms associated with neurodegeneration and may provide avenues for novel therapeutics. Antioxid. Redox Signal. 30, 1450-1499.
\end{abstract}

Keywords: cysteine, sulfhydration, reverse transsulfuration, free radicals, neurodegeneration, redox regulation

\section{Table of Contents}

I. Introduction

II. Free Radicals and Their Generation

A. Reactive oxygen species

1. Hydrogen peroxide

1453

2. Hydroxyl radicals

1453

3. Superoxide anions

1454

4. Hydroperoxyl radical

1454

Reviewing Editors: Vsevolod Belousov, Pamela Maher, Jeremy Marks, Pier Mastroberardino, Rituraj Pal, and Victor Tapias

\footnotetext{
${ }^{1}$ The Solomon H. Snyder Department of Neuroscience, The Johns Hopkins University School of Medicine, Baltimore, Maryland.

Departments of ${ }^{2}$ Pharmacology and Molecular Sciences and ${ }^{3}$ Psychiatry, The Johns Hopkins University School of Medicine, Baltimore, Maryland.
} 
5. Peroxyl radical

6. Singlet oxygen 1454

B. Sources of ROS generation 1454

1. Mitochondria 1454

2. Xanthine oxidoreductases 1455

3. NADPH oxidases 1455

C. Reactive nitrogen species 1455

1. Nitric oxide 1456

2. Peroxynitrite 1456

3. Nitrogen dioxide 1456

D. Reactive sulfur species 1456

E. Effects of ROS and free radicals on cellular components 1457

$\begin{array}{ll}\text { 1. Proteins } & 1457\end{array}$

$\begin{array}{ll}\text { 2. Nucleic acids } & 1457\end{array}$

3. Lipids
4. Carbohydrates
1457

$\begin{array}{ll}\text { 4. Carbohydrates } & 1457\end{array}$

III. Antioxidant Systems in the Brain 1457

A. Small molecules 1457

B. Enzymatic/protein components of redox balance 1458

1. Superoxide dismutases 1458

$\begin{array}{ll}\text { 2. Catalases } & 1459\end{array}$

3. Glutathione peroxidases (enzymes) 1459

4. Peroxiredoxins (enzymes) 1460

IV. Dysregulation of Redox Homeostasis in Neurodegeneration 1460

A. Huntington's disease 1460

1. Oxidative stress in HD 1461

2. Compromised low-molecular-weight antioxidant metabolism in HD 1461

a. Reduced cysteine and GSH levels in HD 1461

b. Reduced ascorbate uptake in HD 1462

3. Elevated production of free radicals in HD 1462

$\begin{array}{lr}\text { a. Activation of NOX enzymes } & 1462\end{array}$

b. Redox metals and HD 1463

c. Defective DNA repair in response to oxidative stress 1463

d. Excessive NMDAR stimulation 1464

e. Impaired antioxidant response pathways in HD 1464

f. Mitochondrial dysfunction in HD 1464

4. Huntingtin as a sensor of oxidative stress 1465

B. Alzheimer's disease $\quad 1465$

1. Amyloid beta, tau, and oxidative stress 1465

2. Mitochondrial dysfunction 1465

3. Transcriptional dysregulation 1466

4. Aberrant nitrosylation 1466

C. Parkinson's disease 1467

$\begin{array}{ll}\text { 1. Redox stress in PD } & 1467\end{array}$

2. Aggregation of $\alpha$-synuclein 1468

3. Mitochondrial dysfunction in PD 1468

4. Iron accumulation in PD 1469

5. Dysregulated calcium signaling in PD 1469

6. Inflammation in PD

7. Dysregulated gasotransmitter signaling in PD 1470

D. Amyotrophic lateral sclerosis 1471

1. ALS and redox imbalance 1471

2. ALS and mutations in SOD1 1472

3. The C9ORF72 model of ALS 1472

E. Autism spectrum disorders 1473

1. Mitochondrial dysfunction and oxidative stress in ASD 1473

2. Abnormal transmethylation, reverse transsulfuration, and vitamin B metabolism in ASD 1473

3. Treatments for ASD 1474

F. The ataxias

1. Autosomal dominant ataxias: spinocerebellar ataxia 1475

2. Autosomal recessive ataxias: ataxia telangiectasia 1475

3. Friedreich's ataxia 1476

G. Aging and redox imbalance 1477

V. Concluding Remarks 1478 


\section{Introduction}

$\mathbf{P}$ RODUCTION OF FREE RADICALS is an inevitable consequence of metabolic processes in cells. This review covers aspects of redox regulation and nodes that have been disrupted in neurodegenerative disorders. In several instances, reactive oxygen species (ROS), reactive nitrogen species (RNS), and reactive sulfur species (RSS) are integral components of signal transduction processes in cells (Fig. 1A-C). These free radicals can modify susceptible amino acid residues on proteins and other cellular components to alter their structure or function. In particular, cysteine residues are highly susceptible to redox modifications. Basal levels of ROS or RNS are always present in cells; however, when they cross a limit, antioxidant defense systems are activated. The body has evolved multiple mechanisms to cope with increased levels of these free radicals and redox-active molecules. When the antioxidant mechanisms of cells cannot handle increased oxidative stress, increased free radicals lead to antioxidant imbalance or oxidative stress. The term "oxidative stress" was classically defined as a disturbance in the prooxidant-antioxidant balance in favor of the former. However, with increasing research on redox signaling, oxidative stress may be better defined as a disruption of redox signaling and control (252). Dysregulated redox regulation has been implicated in the pathology of neurodegenerative disorders. Diseases such as Alzheimer's disease (AD), Parkinson's disease (PD), Huntington's disease (HD), and amyotrophic lateral sclerosis (ALS) are associated with increased levels of
A

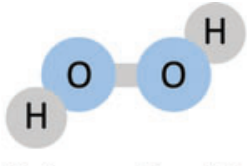

Hydrogen Peroxide

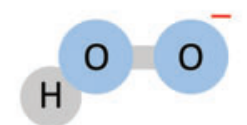

Hydroperoxyl Radical

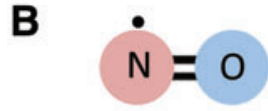

Nitric Oxide

C

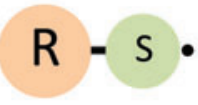

Thiyl Radicals

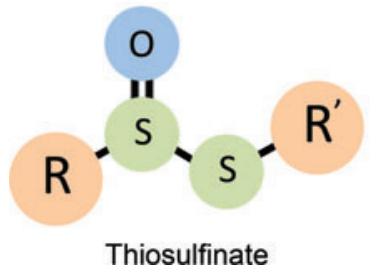

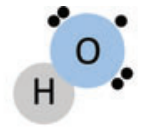

Hydroxyl Radical
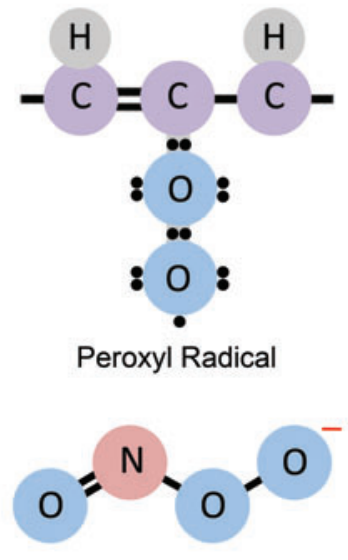

Peroxynitrite

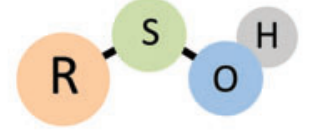

Sulfenic Acids

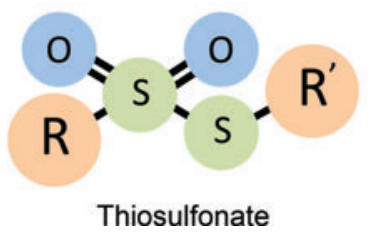

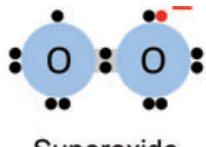

Superoxide

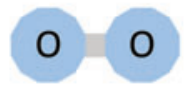

Singlet Oxygen

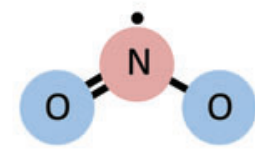

Nitrogen Dioxide

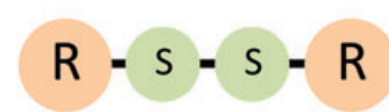

Disulfides

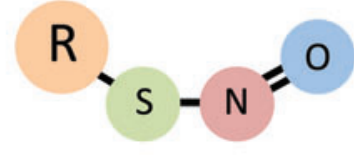

S-Nitrosothiols

FIG. 1. Reactive oxygen, nitrogen, and sulfur species. (A) ROS. ROS are oxygen-derived species and include hydrogen peroxide $\left(\mathrm{H}_{2} \mathrm{O}_{2}\right)$, hydroxyl radical $(\bullet \mathrm{OH})$, superoxide $\left(\mathrm{O}_{2}^{\bullet-}\right)$, hydroperoxyl radical $\left(\mathrm{HO}_{2}^{\bullet}\right)$, peroxyl radical $\left(\mathrm{ROO}{ }^{\bullet}\right)$, and singlet oxygen $\left({ }^{1} \mathrm{O}_{2}\right)$. ${ }^{\circ}$ Refers to an unpaired electron and the negative sign refers to the negative charge on the molecule. (B) RNS. The main RNS include nitic oxide or nitrogen monoxide $\left({ }^{\bullet} \mathrm{NO}\right)$, nitrogen dioxide $\left({ }^{\bullet} \mathrm{NO}_{2}\right)$, and peroxynitrite $\left(\mathrm{ONOO}^{-}\right)$. Nitric oxide is the major RNS and is also the source of several other RNS. Peroxynitrite is generated by the interaction of nitric oxide with superoxide and is highly reactive and toxic to cells. The list is not exhaustive. Other RNS include nitronium cation, nitrosonium cation, nitryl chloride, and nitrosothiols. ${ }^{\circ}$ Refers to an unpaired electron. (C) RSS. The multivalent state of sulfur permits it to form a wide variety of RSS. RSS include disulfides, sulfenic acids, thiyl radicals, thiosulfinate, thiosulfonate, and nitrosothiols. ${ }^{\bullet}$ Refers to an unpaired electron and $\mathrm{R}$ denotes an organic group. RNS, reactive nitrogen species; ROS, reactive oxygen species; RSS, reactive sulfur species. Color images are available online. 
free radicals and oxidative damage, which contribute to disease progression.

Although the brain constitutes only $2 \%$ of total body weight, it consumes $20 \%$ of inhaled oxygen. It is one of the most metabolically active organs in the entire body, accounting, in part, for its generating higher levels of free radicals. Paradoxically, the brain is also one of the most vulnerable tissues to oxidative stress (166). The brain is rich in lipids with high levels of polyunsaturated fatty acids (PUFAs) in neuronal membranes as well as a predominance of membrane components over cytoplasmic components. The double bonds present in PUFAs such as eicosapentanoic (C20:5) and docosahexanoic (C22:6) acids are susceptible to attack by ROS such as hydroxyl radicals $(\bullet \mathrm{OH})$ leading to a chain reaction of lipid peroxidation. Moreover, antioxidant systems in the brain are less efficient than those operating in peripheral organs such as the liver (158). The brain is composed of numerous cell types, whose relative numbers, composition, and functional interactions vary in different regions, leading to the concept of selective neuronal vulnerability (SNV). This concept may explain why specific populations of neurons degenerate selectively in different neurodegenerative diseases and may account for individual predisposition to brain disease (451). In this review, we cover some of the basic principles in redox biology as well as advanced mechanistic concepts that underpin some of the signaling events in neurodegenerative diseases.

\section{Free Radicals and Their Generation}

Free radicals are molecules containing one or more unpaired electrons in their molecular orbitals, capable of existing independently $(104,340,396)$. In biological systems, free radicals are often termed ROS because a large proportion of biologically relevant radicals are oxygen centered. ROS in cells include hydroxyl radical $(\bullet \mathrm{OH})$, superoxide $\left(\mathrm{O}_{2}{ }^{\bullet-}\right)$, singlet oxygen $\left({ }^{1} \mathrm{O}_{2}\right)$, and hydrogen peroxide $\left(\mathrm{H}_{2} \mathrm{O}_{2}\right)$, although $\mathrm{H}_{2} \mathrm{O}_{2}$ is technically not a free radical because it does not have unpaired electrons. RNS are also free radicals that are nitrogen centered and include species such as nitric oxide $\left({ }^{\circ} \mathrm{NO}\right)$, peroxynitrite $\left(\mathrm{ONOO}^{-}\right)$, and nitrogen dioxide $\left({ }^{\circ} \mathrm{NO}_{2}\right)$.
Radicals can form whenever there is a loss or gain of an electron. Thus, a nonradical can lose an electron or gain an electron to become a radical. Whenever electron transfer occurs in a chemical reaction, the reaction is termed a redox reaction. Removal of electrons is oxidation, whereas addition of electrons constitutes reduction. In this section, we describe the major reactive species, their sources in vivo, and their effects on cellular components.

\section{A. Reactive oxygen species}

ROS, as the name implies, involves oxygen (Fig. 1A). In addition to external agents, several enzymatic processes in cells contribute to $\mathrm{H}_{2} \mathrm{O}_{2}$ production (Table 1). Several distinct types of ROS participate in signaling processes.

1. Hydrogen peroxide. $\mathrm{H}_{2} \mathrm{O}_{2}$, although not a free radical, is an ROS that is deleterious to cells at higher concentrations. $\mathrm{H}_{2} \mathrm{O}_{2}$ is the dominant ROS influencing signaling pathways as well as inducing damage under pathophysiological conditions. $\mathrm{H}_{2} \mathrm{O}_{2}$ in turn can give rise to hydroxyl radical $(\bullet \mathrm{OH})$ by either the Fenton reaction or the Haber-Weiss reaction (239, 548).

2. Hydroxyl radicals. $\cdot \mathrm{OH}$ are highly reactive and hence have a very short half-life of the order of $10^{-9} \mathrm{~s}$. They are generated from $\mathrm{H}_{2} \mathrm{O}_{2}$ and act within a few $\mathrm{A}^{\circ}$ of their site of production. They can damage most cellular components, including DNAs, lipids, and carbohydrates. Lipids in cell membranes readily undergo peroxidation when exposed to -OH radicals. Transition metals present in cells can promote formation of $\bullet \mathrm{OH}$ radicals. Transition metals are those elements (manganese, iron, cobalt, zinc, and molybdenum) that belong to the $\mathrm{d}$ series (Groups 3-12 of the periodic table), with a partially filled d subshell, or that can give rise to cations with an incomplete d subshell (100). Electrons in the d orbital can participate in bond formation (alongside the $4 \mathrm{~s}$ electrons) such that the element can exist in different oxidation states and are redox active. For instance, iron and copper are redox active and can transfer an electron by alternating between two redox states $\left(3^{+} / 2^{+}\right.$in the case of iron

\section{Table 1. Enzymes Generating Free Radicals}

Enzyme generating free radicals

Xanthine oxidase

NADPH oxidases

Aldehyde oxidase

Monoamine oxidase

Cytochrome $\mathrm{P} 450$ reductase

SOD1-3

D-aminoacid oxidase

Mitochondrial electron transport complexes

2-Oxoglutarate dehydrogenase (OGDH) complex

L- $\alpha$-hydroxyacid oxidase

Myeloperoxidase

Nitric oxide synthases
Reaction catalyzed

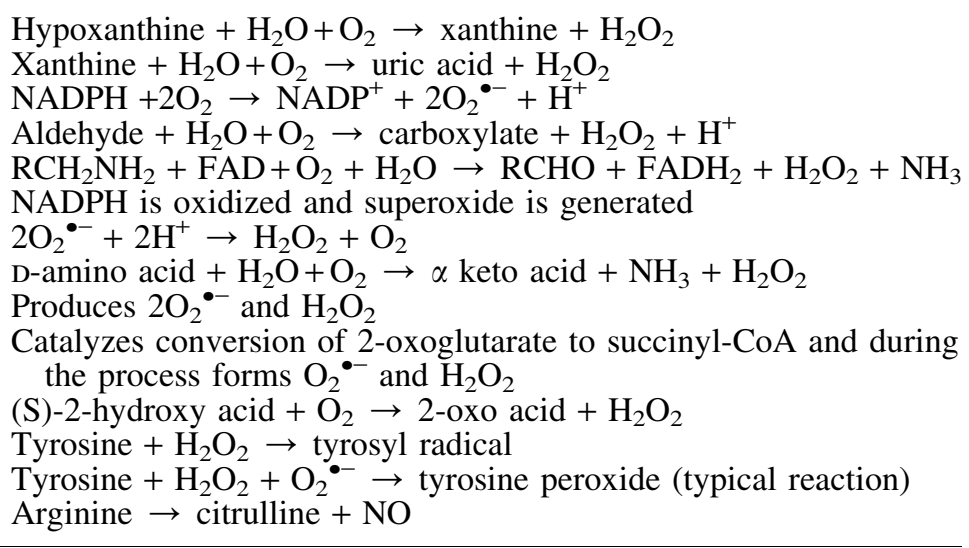

FAD, flavin adenine dinucleotide; $\mathrm{H}_{2} \mathrm{O}_{2}$, hydrogen peroxide; $\mathrm{NADPH}$, nicotinamide adenine dinucleotide phosphate; SOD, superoxide dismutase. 
and $2^{+} / 1^{+}$for copper). These ions play important roles in biological redox reactions but can also be toxic participants in free radical generating processes such as the Fenton reaction. Thus, the reaction between ferrous $\left(\mathrm{Fe}^{2+}\right)$ ions and $\mathrm{H}_{2} \mathrm{O}_{2}$ produces $\bullet \mathrm{OH}$ and $\mathrm{HO}_{2}$ to oxidize lipids, proteins, and DNAs via the reactions.

$$
\begin{aligned}
& \mathrm{Fe}^{2+}+\mathrm{H}_{2} \mathrm{O}_{2} \rightarrow \mathrm{Fe}^{3+}+\cdot \mathrm{OH}+\mathrm{OH}^{-} \\
& \mathrm{Fe}^{3+}+\mathrm{H}_{2} \mathrm{O}_{2} \rightarrow \mathrm{Fe}^{2+}+\mathrm{HOO}^{\bullet}+\mathrm{H}^{+}
\end{aligned}
$$

3. Superoxide anions. $\mathrm{O}_{2}{ }^{--}$are generated predominantly by the mitochondria and are by-products of aerobic metabolism as a result of one electron reduction of molecular oxygen by enzymes in the mitochondrial respiratory chain. $\mathrm{O}_{2}{ }^{\bullet-}$ produced by the mitochondria are capable of reducing $\mathrm{Fe}^{3+}$ to $\mathrm{Fe}^{2+}$ by the Haber-Weiss reaction (262).

$$
\mathrm{O}_{2}^{\bullet-}+\mathrm{Fe}^{3+} \rightarrow \mathrm{Fe}^{2+}+\mathrm{O}_{2}
$$

In addition, $\mathrm{O}_{2}{ }^{--}$can react with ${ }^{\bullet} \mathrm{NO}$ to produce $\mathrm{ONOO}^{-}$. Other sources of $\mathrm{O}_{2}{ }^{--}$include enzymes in the NADPH (nicotinamide adenine dinucleotide phosphate) oxidase (NOX) family, xanthine oxidase, autoxidation reactions of reduced flavins, quinones, metal ions, metalloproteins, and exposure to ionizing radiation or photochemical irradiation.

4. Hydroperoxyl radical. Hydroperoxyl radical $\left({ }^{\bullet} \mathrm{HO}_{2}\right)$ is the protonated form of $\mathrm{O}_{2}{ }^{\bullet-}$ and is also termed as perhydroxyl radical. About $0.3 \%$ of $\mathrm{O}_{2}{ }^{\bullet-}$ present in the cell cytosol exists in the protonated form (122). ${ }^{\bullet} \mathrm{HO}_{2}$ can induce lipid peroxidation and also mediate tumor formation.

5. Peroxyl radical. The primary pathway of peroxyl radical, $\mathrm{ROO}^{\circ}$, formation in biological systems is autoxidation $v i z$. lipid peroxidation $(419,480)$. Lipid peroxidation occurs when free radicals attack lipids containing carbon/carbon double bond(s), especially PUFAs. In addition, lipid peroxidation can also be caused by enzymes such as lipoxygenases, cyclooxygenases, and cytochrome P450 (19). ROO ${ }^{\circ}$ radicals participate in a chain reaction through which PUFAs are attacked and generate more radicals. These radicals can damage cells by reacting with diverse macromolecules, such as lipids, carbohydrates, proteins, nucleic acids, and enzymes. The effects of $\mathrm{ROO}^{\bullet}$ are neutralized by vitamin $\mathrm{E}$ or $\alpha$ tocopherol, which is a major chain breaking antioxidant.

6. Singlet oxygen. ${ }^{1} \mathrm{O}_{2}$ is formed when photosensitizers such as chlorophyll absorb light energy and transfer it to oxygen. ${ }^{1} \mathrm{O}_{2}$ can be beneficial as well as harmful to cells (371). For example, ${ }^{1} \mathrm{O}_{2}$ can be bactericidal or antimicrobial (157), but it can also cause cell death by necrosis and apoptosis (373). ${ }^{1} \mathrm{O}_{2}$ can damage DNA and proteins (62). The photoaging-linked mitochondrial common deletion is mediated by ${ }^{1} \mathrm{O}_{2}$ (44). The common deletion refers to a $4977 \mathrm{bp}$ deletion, which is considered to be a marker for mutations in the mitochondrial genome. Antioxidants, such as $\alpha$ tocopherol and $\beta$-carotene, and thiols, such as ergothioneine and methionine, scavenge ${ }^{1} \mathrm{O}_{2}(128,139,279)$.

\section{B. Sources of ROS generation}

The generation of free radicals is positively correlated with metabolic rate and is inversely proportional to life span (9). Several organelles and physiological processes contribute to the generation of ROS. A few of the major ROS-generating sources are discussed below.

1. Mitochondria. Among the organelles generating ROS, the mitochondria are the major contributors that, if not sequestered, can damage the organelle itself. Mitochondria are the sites of aerobic respiration via oxidative phosphorylation (OXPHOS), accounting for $90 \%$ of oxygen taken up by cells and provide about $80 \%$ of the energy requirements, the remaining $20 \%$ being met by glycolysis (378). Mitochondria are bilayered organelles, with outer and inner membranes. Five multiprotein complexes (designated complex I-V) constitute the mitochondrial OXPHOS system (98). Electrons are relayed from NADH, an intermediate of the Krebs cycle, to NADH coenzyme Q reductase (complex I), which passes them onto ubiquinone or coenzyme $\mathrm{Q}$, which also receives electrons from succinate dehydrogenase $\mathrm{SDH}$; complex II). Coenzyme Q passes electrons to complex III (cytochrome bc1), which passes them to cytochrome $\mathrm{C}$, which transfers them to complex IV (cytochrome C oxidase) that in turn uses these electrons to reduce molecular oxygen to water (Fig. 2).

$\mathrm{O}_{2}{ }^{\bullet-}$ is generated predominantly by complexes I and III of the mitochondria, especially when there is an abundance of respiratory substrates derived from the diet $(358,411)$. Complex I is a large multisubunit membrane-bound complex, comprising at least 45 polypeptides, transferring electrons from NADH to the cofactor of complex I, flavin mononucleotide (FMN), to quinone via a series of proteins harboring iron/sulfur $(\mathrm{Fe}-\mathrm{S})$ clusters $(75,411)$. Although early studies provided estimates of $\mathrm{O}_{2}{ }^{\bullet-}$ production, these studies were conducted in vitro on isolated mitochondria or mitochondrial fractions (50). In cells, $\mathrm{O}_{2}{ }^{\bullet-}$ is acted on by superoxide reductases and dismutases to produce $\mathrm{H}_{2} \mathrm{O}_{2}$ (469). Thus, accurate in vivo measurement of $\mathrm{O}_{2}{ }^{\bullet-}$ is difficult (358). $\mathrm{O}_{2}{ }^{--}$ production by complex I can occur by different modes. Briefly, the first mode is operational during the conventional forward electron transport when FMN accepts electrons from $\mathrm{NADH}$ and is reduced. Here two electrons are transferred from NADH to reduce coenzyme Q. Another mode of $\mathrm{O}_{2}{ }^{\bullet-}$ production occurs during reverse electron transport. Here electrons flow backward through complex I to FMN from where they can reduce NAD ${ }^{+}$to $\mathrm{NADH}$ and also cause $\mathrm{O}_{2}{ }^{\bullet-}$ formation $(97,358)$. Complex III is composed of 11 polypeptides, three hemes and an Fe-S center, and transfers electrons from coenzyme $\mathrm{Q}$ pool to cytochrome $\mathrm{c}$ and can also be a source of $\mathrm{O}_{2}{ }^{\bullet-}$. The mechanisms of $\mathrm{O}_{2}{ }^{\bullet-}$ production by complex I and III have been covered by several detailed reviews $(53,97,358)$.

Most intracellular ROS owe their origin to $\mathrm{O}_{2}{ }^{\bullet-}$, which is converted to $\mathrm{H}_{2} \mathrm{O}_{2}$ by the action of catalase or react with ${ }^{\bullet} \mathrm{NO}$ to form $\mathrm{ONOO}^{-}$. Oxidative stress can damage mitochondrial components such as mitochondrial DNA (mtDNA) and proteins in addition to nuclear DNA, which can further inflict damage $(144,424)$. The mitochondrial genome is not protected by histones and is highly susceptible to damage due to its proximity to the electron transport chain (ETC). Similar 

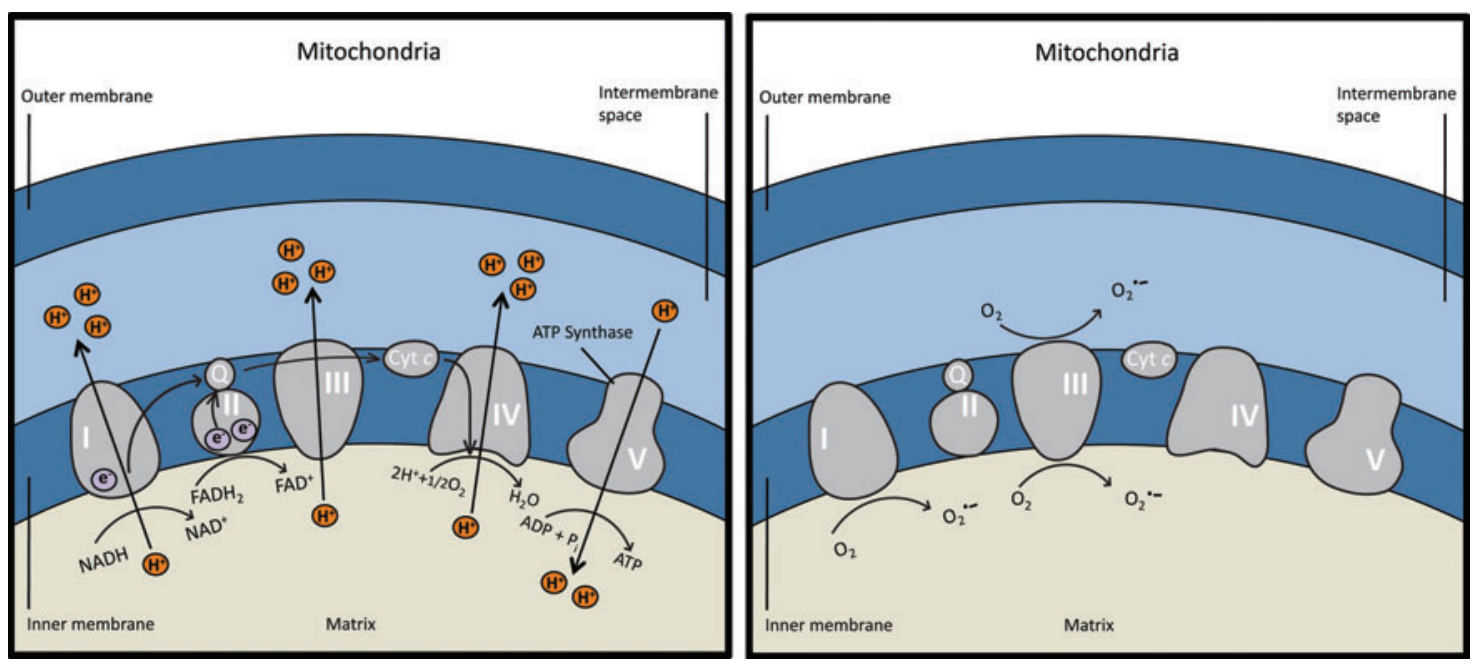

FIG. 2. The mitochondrial ETC and sites of ROS production. The ETC, functioning in oxidative phosphorylation, resides on the inner mitochondrial membrane and is composed of five multiprotein complexes, designated I-V. NADH, an intermediate of the Krebs cycle transfers electrons to complex I (NADH coenzyme Q reductase), which is transported to ubiquinone/coenzyme $\mathrm{Q}$, which also receives electrons from succinate dehydrogenase (complex II). Coenzyme Q relays electrons to complex III (cytochrome bc1), which passes them on to cytochrome C and then to complex IV (cytochrome C oxidase). This enzyme transfers electrons to oxygen (which is the terminal electron acceptor and is reduced to water), while pumping protons across the membrane. The proton motive force is utilized by the $\mathrm{F}_{0} \mathrm{~F}_{1}$ ATP synthase complex (often referred to as complex V) to catalyze the formation of ATP from ADP. During the process of electron transports, those that leak from the ETC can react with molecular oxygen in the mitochondrial matrix to form superoxide, hydrogen peroxide, and hydroxyl radicals and increase oxidative stress. Complex I and III are sites of superoxide production. Complex I produces elevated levels of ROS when the NADH/NAD ${ }^{+}$ratio is high or when coenzyme $\mathrm{Q}$ is low in combination with low $\mathrm{ATP}$ synthesis. ATP, adenosine triphosphate; ETC, electron transport chain. Color images are available online.

levels of oxidants induce more lesions in mtDNA compared with nuclear DNA $(325,524)$. On an average a single mammalian cell is expected to undergo 2000-10,000 depurination (hydrolysis of the $\mathrm{N}$-glycosyl linkage connecting a purine base to the deoxyribose sugar is cleaved, producing an abasic site) events per generation. In terminally differentiated cells such as neurons, $\sim 10^{8}$ such events are expected to occur (306). Damage to mtDNA prevents optimal expression of proteins in the ETC leading to a toxic cycle of free radical generation and mitochondrial malfunction, which culminates in neuronal death (518). It is not surprising that the process of respiration has evolved to be carried out by a distinct organelle to shield the other cellular compartments such as the nucleus.

2. Xanthine oxidoreductases. In addition to external agents and the mitochondria, several cellular enzymes contribute to $\mathrm{H}_{2} \mathrm{O}_{2}$ production. Xanthine oxidoreductase (XOR) catalyzes the conversion of hypoxanthine to xanthine and further to uric acid during purine metabolism, generating $\mathrm{H}_{2} \mathrm{O}_{2}$ in the process. XOR is a homodimer, with each subunit comprising a molybdopterin cofactor, two iron/sulfur centers and a flavin adenine dinucleotide (FAD) cofactor. Expression of XOR is regulated by oxygen tension, cytokines, and glucocorticoids (45). XORs exist either as the dehydrogenase form (xanthine dehydrogenase $[\mathrm{XDH}]$ ) or the oxidase form (XO), generated from the reversible oxidation of cysteine residues or by irreversible proteolytic cleavage (284, 368, 486). Electrons are relayed from xanthine to oxygen and $\mathrm{NAD}^{+}$, respectively, yielding $\mathrm{O}_{2}^{\bullet-}, \mathrm{H}_{2} \mathrm{O}_{2}$, and NADH. In addition, XOR can generate $\mathrm{O}_{2}^{\bullet-}$ via $\mathrm{NADH}$ oxidase activity and can produce ${ }^{\bullet} \mathrm{NO}$ via nitrate and nitrite reductase activities (45).

3. NADPH oxidases. The NOX family of enzymes are protein complexes that utilize cytosolic NADPH to reduce oxygen to $\mathrm{O}_{2}{ }^{\bullet-}$ anion $(287,321,365)$. Each of these enzymes contains a core catalytic subunit (NOX) and regulatory subunits that are located in the cytosolic and membranous compartments. Neutrophils and other phagocytic cells were the first cells shown to possess NOX activity, which was utilized for defense against microorganisms. The catalytic subunit of this enzyme gp91phox or NOX2 is now recognized to be one of seven known members constituting the family namely, NOX1, NOX2, NOX3, NOX4, NOX5, dual oxidase 1 (DUOX1), and DUOX2 (539). These enzymes transfer an electron from NADPH to $\mathrm{O}_{2}$, forming $\mathrm{O}_{2}{ }^{\bullet-}$, which in turn is converted to $\mathrm{H}_{2} \mathrm{O}_{2}$. The physiologic functions of these enzymes include host defense, regulation of gene expression, and cell differentiation. It is becoming increasing clear that NOX activity is elevated in several neurodegenerative conditions and inhibition or genetic deletion of these enzymes has therapeutic benefits $(37,94,95,377,476,544)$.

Several other enzymes such as cytochrome $\mathrm{P} 450$ reductase also produce free radicals and are listed in Table 1.

\section{Reactive nitrogen species}

Although redox imbalance was selectively attributed to ROS in the past, it is being increasingly clear that other free 
radical species such as RNS also contribute to fluctuations in redox homeostasis (Fig. 1B).

1. Nitric oxide. ${ }^{\bullet} \mathrm{NO}$ is the predominant RNS that plays central roles in cell signaling processes $(46,236){ }^{\bullet} \mathrm{NO}$ is also the precursor of other RNS such as peroxynitrites. In mammals, ${ }^{\bullet} \mathrm{NO}$ is produced by nitric oxide synthases (NOSs) from L-arginine. ${ }^{\circ} \mathrm{NO}$ functions as a second messenger to regulate numerous physiologic processes, ranging from vascular function and smooth muscle contraction/dilation to inflammation and neuroplasticity.

2. Peroxynitrite. $\mathrm{ONOO}^{-}$is a highly reactive radical formed by the interaction of ${ }^{\bullet} \mathrm{NO}$ and $\mathrm{O}_{2}{ }^{\bullet-}$ and mediates several of the toxic effects of these radicals in cells (408, 409). The radical is short lived and causes extensive cytotoxicity. $\mathrm{ONOO}^{-}$affects mitochondrial function and triggers cell death via oxidation and nitration reactions. $\mathrm{ONOO}^{-}$ formation leads to decreased bioavailability of ${ }^{\bullet} \mathrm{NO}$ and therefore affects most processes regulated by ${ }^{\bullet} \mathrm{NO}$.

3. Nitrogen dioxide. Once believed to be predominantly an environmental pollutant, nitrogen dioxide has been reported to be produced endogenously by enzymes such as myeloperoxidases (318). Nitrogen dioxide is derived from ${ }^{\bullet} \mathrm{NO}$ and oxygen, a reaction that takes place most efficiently in a hydrophobic environment. ${ }^{\bullet} \mathrm{NO}_{2}$ can trigger lipid peroxidation reactions and cell death (269). More recently, it was shown that the in vivo formation of 3-nitrotyrosine depends on the availability of ${ }^{\bullet} \mathrm{NO}_{2}$ radicals. Nitrogen dioxide can deplete endogenous antioxidants such as ascorbic acid, alpha-tocopherol, and bilirubin, which help scavenge this radical (207).

\section{Reactive sulfur species}

For many years the redox field largely focused on reactive oxygen and nitrogen species. Sulfur was considered to be only a building block of proteins and small molecules such as the reduced form of glutathione (GSH). Sulfur compounds such as sulfur dioxide $\left(\mathrm{SO}_{2}\right)$ and hydrogen sulfide $\left(\mathrm{H}_{2} \mathrm{~S}\right)$ were considered to be toxic environmental components. However, recent advances in the field of sulfur biology have established RSS as signaling molecules, and it is becoming increasingly evident that RSS also play significant roles in redox reactions in vivo $(177,187)$. Oxidation products of the thiol groups of cysteine residues, the disulfides, sulfenic acids, sulfinic acids, and sulfonic acids, were known due to studies of thiol oxidation in proteins. RSS can be generated from thiols by reaction with oxidizing agents such as $\mathrm{H}_{2} \mathrm{O}_{2},{ }^{1} \mathrm{O}_{2}, \mathrm{ONOO}^{-}$, and $\mathrm{O}_{2}{ }^{\bullet-}$. The effect of endogenous and dietary sulfur containing thiols such as GSH, cysteine, and ergothioneine in redox buffering and mitigation of oxidative stress was also known. The role of cysteine modifications such as glutathionylation was also gaining importance and continues to be a major field of study (173-175). The large number of RSS owes its origin to the multivalent oxidation states of sulfur, ranging from -2 , in $\mathrm{H}_{2} \mathrm{~S}$, to +6 , in sulfate $\left(\mathrm{SO}_{4}{ }^{2-}\right)$. The concept of $\mathrm{RSS}$ was first introduced in 2001 and included thiyl radicals $\left(\mathrm{RS}^{\bullet}\right)$, sulfenic acids (RSOH), disulfides (RSSR), thiosulfinate [RS(O)SR], thiosulfonate $\left[\mathrm{RS}(\mathrm{O})_{2} \mathrm{SR}\right]$, and S-nitrosothiols (SNT) (178) (Fig. 1C). These were proposed to be formed during conditions of oxidative stress and act as oxidizing agents themselves.

The mitochondria are a rich source of RSS during sulfide oxidation. Although initial studies focused only on thiosulfate and sulfate, other sulfur species such as glutathione persulfide (GSSH) are generated during mitochondrial functions $(304,348)$. Sulfide oxidation in the mitochondria is catalyzed by sulfide quinone oxidoreductase, a flavoprotein, via a two-electron oxidation of $\mathrm{H}_{2} \mathrm{~S}$, forming a persulfide intermediate during the reaction $(241,304)$. The persulfide is transferred to a low-molecular-weight acceptor such as GSH. The released electrons are relayed to complex III of the ETC by ubiquinone. The GSSH formed during the process can be acted on by enzymes such as persulfide dioxygenase to form sulfite. Alternatively, GSSH can be utilized in a sulfurtransferase reaction by rhodanese, which transfers the sulfane sulfur to sulfite, forming thiosulfate in the process. The sulfite formed is oxidized by enzymes such as eosinophil peroxidase, prostaglandin $\mathrm{H}$ synthase, or myeloperoxidase to yield RSS, sulfite anion radical, peroxymonosulfate radical, and sulfate radicals, which can damage proteins in cells (416). Sulfur amino acids are major targets of redox modifications and thus the identification of an increasing number of sulfurderived radicals adds additional layers of complexity in redox signaling processes (457).

Recently, the gasotransmitter, $\mathrm{H}_{2} \mathrm{~S}$, has been identified as a bona fide signaling molecule. $\mathrm{H}_{2} \mathrm{~S}$, derived from cysteine, has pleiotropic roles in cell function, ranging from inflammation to cardiovascular functions. $\mathrm{H}_{2} \mathrm{~S}$ functions as an endothelialderived relaxation factor and like ${ }^{\bullet} \mathrm{NO}$ increases cyclic GMP in cells. Like ${ }^{\bullet} \mathrm{NO}, \mathrm{H}_{2} \mathrm{~S}$ participates in physiological pathways in the brain. One of the first identified neural functions of $\mathrm{H}_{2} \mathrm{~S}$ was the regulation of the N-methyl-D-aspartate (NMDA) receptor (NMDAR) and induction of long-term potentiation, suggesting a role as a neuromodulator (2) $\mathrm{H}_{2} \mathrm{~S}$ has cytoprotective functions and promotes neuronal survival in several instances. One of the modes by which $\mathrm{H}_{2} \mathrm{~S}$ signals is via a posttranslational modification of reactive cysteine residues designated sulfhydration or persulfidation, which is analogous to nitrosylation mediated by ${ }^{\bullet} \mathrm{NO}(20,360,382,384-386,484)$. $\mathrm{H}_{2} \mathrm{~S}$ participates in stress signaling caused by inflammatory stimuli to mediate cytoprotective effects. When macrophages are stimulated with tumor necrosis factor $\alpha(\mathrm{TNF} \alpha)$, p65 subunit of the transcription factor, nuclear factor kappa B, is sulfhydrated at Cys38, which stimulates its interaction with the ribosomal protein Rps 3 and increases its binding to promoters of prosurvival genes to reduce inflammation (466). In a vast majority of proteins, sulfhydration and nitrosylation have opposing effects and frequently occur on the same cysteine residue in a mutually exclusive manner. While nitrosylation typically reduces the reactivity of the cysteine thiols, sulfhydration increases their reactivity, rendering them more nucleophilic. Glyceraldehyde 3-phosphate dehydrogenase (GAPDH), which is the key regulatory enzyme of glycolysis and a regulator of transcription, can be modified by sulfhydration and nitrosylation at Cys150. The glycolytic activity is enhanced sevenfold by sulfhydration (360). Mice lacking cystathionine $\gamma$-lyase (CSE), the biosynthetic enzyme for $\mathrm{H}_{2} \mathrm{~S}$, have decreased GAPDH activity in the liver. On the contrary, nitrosylation at the same cysteine residue inhibits GAPDH activity, which causes it to translocate to the nucleus and to modulate transcription $(209,465)$. Sulfhydration is more 
prevalent than nitrosylation and $\sim 25 \%$ of GAPDH is modified in hepatic lysates (360). These reciprocal effects have also been observed in the brain and are discussed later. Thus, $\mathrm{H}_{2} \mathrm{~S}$ can modulate several aspects of cellular physiology.

\section{E. Effects of ROS and free radicals on cellular components}

ROS affect several cellular components, including proteins, nucleic acids, lipids, and carbohydrates.

1. Proteins. ROS can cause oxidation of side chains of constituent amino acids in proteins, which can result in protein carbonylation (482). The thiol group $(-\mathrm{SH})$ of cysteine residues is readily oxidized when exposed to ROS. The-SH group of cysteine residues on amino acids can be oxidized to sulfenic (-SOH), which can then be further oxidized to sulfinic $\left(-\mathrm{SO}_{2} \mathrm{H}\right)$ or sulfonic $\left(-\mathrm{SO}_{3} \mathrm{H}\right)$ acid groups or form interor intramolecular disulfide bonds (6). Oxidation of cysteine residues can also facilitate other post-translational modifications such as glutathionylation and sulfhydration, which play important signaling roles in cells (150). Methionine can also be oxidized to methionine sulfoxide (MetO) both during normal and stress conditions $(326,483)$. Recent studies show that the molecule interacting with CasL proteins, which harbor flavin-monooxygenase domain with an NADPHdependent methionine sulfoxidase activity, oxidizes methionine residues in vivo $(231,296)$. In addition to these changes, free radicals such as $\mathrm{ONOO}^{-}$can elicit protein tyrosine nitration, which can participate in both physiological and pathophysiological processes $(29,408)$.

2. Nucleic acids. The DNA base, guanine, is prone to oxidative damage and is converted to 8-oxoguanine, which pairs with adenine instead of cytosine, leading to mutations. More complex modifications such as strand crosslinking and cyclization of nucleotides also occur when DNA is exposed to oxidants and have been extensively discussed $(61,63,152)$.

3. Lipids. Lipids undergo a modification generally termed "lipid peroxidation" through free radical chain reactions (478, 555). Lipids such as cholesterol esters, phospholipids, and triglycerides are particularly vulnerable to modification by free radicals as they comprised PUFAs. Lipid peroxidation results in oxidation products such as reactive aldehydes of which 4-hydroxynonenal (4HNE), malondialdehyde (MDA), and acrolein have been extensively studied. These compounds can react with proteins and other cellular components to modulate their function. 4HNE and MDA have been recognized as markers of lipid peroxidation.

4. Carbohydrates. ROS and RNS mediate damage to carbohydrate components of the cells too, causing several undesirable effects such as oxidative degradation or depolymerization of polysaccharides via scission of carbohydrate chains (135). In rheumatoid arthritis, ROS generated by neutrophils cause fragmentation of hyaluronan, a polymer of the disaccharide [-D-glucuronic acid- $\beta-1,3-\mathrm{N}$-acetyl-Dglucosamine- $\beta$-1,4-] (185).

\section{Antioxidant Systems in the Brain}

As summarized above, a diverse array of free radicals and related damaging species are generated in vivo both during normal metabolism and during pathological conditions. To counteract them, a large arsenal of antioxidants and enzymes are harnessed by cells. When these countermeasures are not successful, redox balance is perturbed and oxidative stress ensues. However, a note of caution is to be mentioned. Antioxidants may not always be beneficial, especially under conditions where the production of free radicals is necessary for cell function as in the case of immune system and during certain aspects of cellular differentiation. Regardless, defense molecules can be broadly categorized into small-molecule antioxidants and protein/enzymatic antioxidants.

\section{A. Small molecules}

Several low-molecular-weight compounds such as cysteine, ascorbate (vitamin C), GSH, ergothioneine, $\alpha$-tocopherol (vitamin E), carotenoids, uric acid, and bilirubin function as antioxidants (Fig. 3) (73). Several of these molecules are synthesized endogenously, whereas others are derived from the diet. Among these molecules, some are lipophilic and are effective in preventing lipid-rich components of cells. For instance, bilirubin, produced by the enzyme biliverdin reductase (BVR), and vitamin E are potent inhibitors of lipid peroxidation $(359,463,487)$. Several of these small molecules form a redox couple (a pair of molecules that can be interconverted by the addition or loss of electrons are referred to as a redox couple or pair) with their oxidized forms, for instance, GSH/GSSG and CysSH/Cys-SS, where GSSG and Cys-SS are the oxidized forms of GSH and cysteine, respectively. Other redox duos in cells include the NADPH/ $\mathrm{NADP}^{+}$and NADH/NAD ${ }^{+}$couples. In cells, there is no single or uniform redox state, as various subcellular compartments have different redox systems operating at distinct set points. Thus, the activity of any one particular redox regulating system may not be representative of the overall scenario. These redox systems are not in equilibrium with each other (264). The redox potentials (the electromotive force $E_{\mathrm{h}}$ or inherent tendency to accept or donate electrons, which is expressed in millivolts) of these redox couples vary within different cellular compartments and conditions. For instance, the $E_{\mathrm{h}}$ of the GSH/GSSG couple is approximately from -260 to $-200 \mathrm{mV}$ in cultured cells. $E_{\mathrm{h}}$ for the GSH/GSSG couple is calculated using the Nernst equation: $E_{\mathrm{h}}=E_{\mathrm{o}}+\mathrm{RT} / n \mathrm{~F} \ln$ $\left[(\mathrm{GSSG}) /(\mathrm{GSH})^{2}\right]$, where $E_{\mathrm{o}}$ is the standard potential, $\mathrm{R}$ is the gas constant, $\mathrm{T}$ is the absolute temperature, $n$ (here 2 ) is the number of electrons transferred, and F is the Faraday's constant. The concentrations of GSH and GSSG can be estimated by methods such as high-performance liquid chromatography. In the plasma of mammals, this value is about $-128 \pm 9 \mathrm{mV}$ (253). In the endoplasmic reticulum (ER), the redox environment is more oxidized than in the cytoplasm (234). The ratio of GSH to GSSG within the secretory pathway ranges from $1: 1$ to $3: 1$. The GSSG/GSH couple is also more oxidized during aging and during pathological conditions (445). The major redox couple in the extracellular milieu is the CysSH/Cys-SS pair. The redox potential of this redox duo is about $-80 \mathrm{mV}$ in normal healthy humans, a value that is significantly more oxidized than that of the GSSG/ GSH couple, which in turn is significantly more oxidized than 


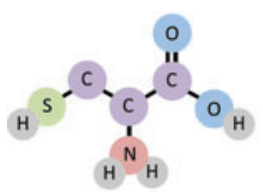

Cysteine

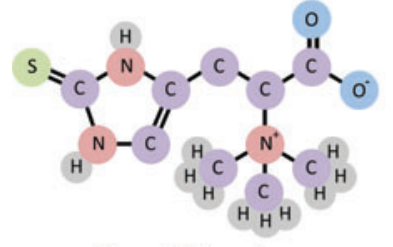

Ergothioneine

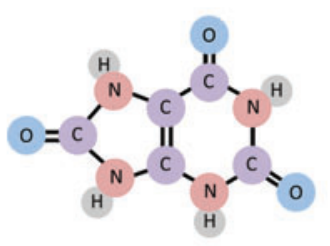

Uric Acid

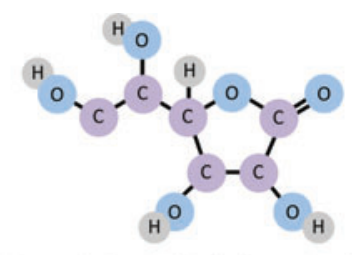

Ascorbic Acid (Vitamin C)
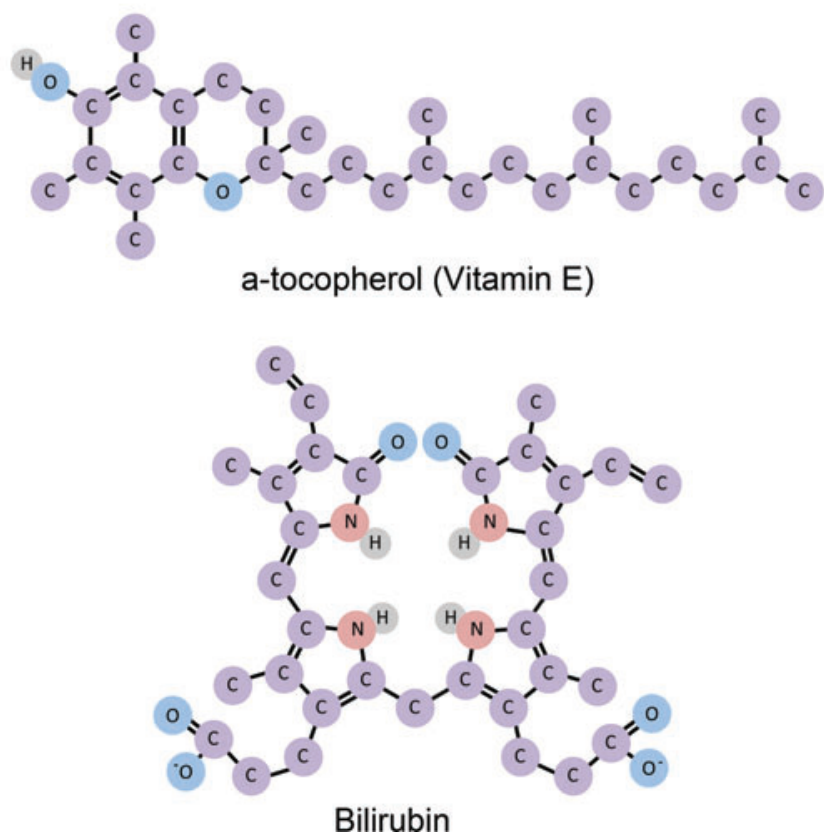

FIG. 3. Small-molecule antioxidants in cells. Cells utilize a wide variety of molecules to counteract redox imbalance, which includes amino acids and metabolites, including but not limited to cysteine, glutathione, bilirubin, and uric acid, which can be endogenously produced. Of these, bilirubin and vitamin $\mathrm{E}$ are lipophilic and protect lipid components of cells. In addition, antioxidants derived from the diet such as vitamin $\mathrm{C}, \mathrm{E}$, and ergothioneine help mitigate redox stress. Color images are available online. the NADPH/NADP ${ }^{+}$system, which oscillates in the -300 to $-400 \mathrm{mV}$ range. Similar to the GSSG/GSH dynamics, the redox potential of the CysSH/Cys-SS redox couple also varies between various cellular compartments and during response to oxidative stress or pathological conditions (264). Emerging evidence suggests that measurements of redox states in cells may be more complicated than previously believed. Cells have multiple compartments and organelles with different redox changes controlled temporally and spatially. Very often, measurements of redox potential using whole cell extracts are considered cytosolic redox potentials, which is not the case. Ex vivo oxidation during sample handling can also contribute to variation in measured values of redox potential as has been reported in the case of the ER (130). Genetically encoded redox sensors that can be targeted to specific cellular compartments can provide information on the redox states of specific compartments. Interestingly, the redox potentials reported by these methods are much more negative (about $-320 \mathrm{mV}$ for cytosolic glutathione redox potentials) in a variety of models due to rapid removal of GSSG into the luminal compartments $(119,353)$.

A systematic analysis of changes in these redox couples in various disease conditions is yet to be conducted. Although the redox potential provides information on redox changes or disturbances, a living cell is complex, with several competing pathways, and kinetic pathways and rates associated with reactions, contributing to whether a particular reaction will occur or not. These aspects have been discussed in detail elsewhere $(153,402,537)$.

\section{B. Enzymatic/protein components of redox balance}

Proteins involved in antioxidant defense can be classified as direct or indirect antioxidant enzymes (514). The direct antioxidant proteins neutralize or scavenge free radicals and include superoxide dismutases (SODs), catalase, glutathione peroxidases, and peroxiredoxins (73) (Table 2). The indirect antioxidant enzymes contribute to reduction of free radicals by indirect means. An example is glutamate-cysteine ligase and CSE, which are required for biosynthesis of GSH and cysteine, respectively $(145,382)$. The antioxidant enzymes play significant roles in maintenance of redox balance and probably constitute the most important arm of antioxidant defense mechanisms. The efficacy of small molecules such as GSH requires the action of such enzymes to exert their effects. Dysregulation of these antioxidant enzymes is frequently encountered in neurodegeneration, which contributes to pathophysiology and disease progression. A few of the major direct antioxidant enzymes are discussed below.

1. Superoxide dismutases. SODs are metalloenzymes that scavenge $\mathrm{O}_{2}{ }^{\bullet-}$ by catalyzing the dismutation of two molecules of $\mathrm{O}_{2}{ }^{\bullet-}$ into one molecule each of $\mathrm{O}_{2}$ and of $\mathrm{H}_{2} \mathrm{O}_{2}$ (341). Three SODs have been identified in mammals so far: copper zinc superoxide dismutase (CuZnSOD/SOD1), manganese superoxide dismutase (MnSOD/SOD2), and extracellular superoxide dismutase (ECSOD/SOD3). SOD1 functions as a homodimer, with each monomer being $\sim 19 \mathrm{kDa}$ and binding an atom each of copper and zinc (286). SOD1 is 
Table 2. Redox Regulating EnZymes

\begin{tabular}{|c|c|}
\hline Enzyme/protein & Function \\
\hline SOD1-3 & Dismutates superoxide to $\mathrm{H}_{2} \mathrm{O}_{2}$ \\
\hline Catalase & Decomposes $\mathrm{H}_{2} \mathrm{O}_{2}, 2 \mathrm{H}_{2} \mathrm{O}_{2} \rightarrow 2 \mathrm{H}_{2} \mathrm{O}+\mathrm{O}_{2}$ \\
\hline \multirow[t]{4}{*}{ Glutathione peroxidases (GPx1-8) } & Utilizes glutathione to reduce $\mathrm{H}_{2} \mathrm{O}_{2}$ to water \\
\hline & $2 \mathrm{GSH}+\mathrm{H}_{2} \mathrm{O}_{2} \rightarrow \mathrm{GSSG}+2 \mathrm{H}_{2} \mathrm{O}$ \\
\hline & Reduces lipid hydroperoxides to corresponding alcohols \\
\hline & Of these, Gpx 4 is unique as it reduces lipid hydroperoxides within the membrane \\
\hline \multirow[t]{2}{*}{ Peroxiredoxins (PRDX1-6) } & Reduces $\mathrm{H}_{2} \mathrm{O}_{2}$ to water \\
\hline & $\operatorname{Prx}($ reduced $)+\mathrm{H}_{2} \mathrm{O}_{2} \rightarrow \operatorname{Prx}($ oxidized $)+2 \mathrm{H}_{2} \mathrm{O}$ \\
\hline \multirow[t]{3}{*}{ Thioredoxins (TRXN1-2) } & Catalyzes reduction of oxidized cysteine residues on proteins \\
\hline & For example, oxidized peroxiredoxin is regenerated by thioredoxins \\
\hline & $\operatorname{Prx}($ oxidized $)+\operatorname{Trx}($ reduced $) \rightarrow \operatorname{Prx}($ reduced $)+\operatorname{Trx}($ oxidized $)$ \\
\hline Sulfiredoxins (Srx) & $\begin{array}{l}\text { Converts cysteine sulfenic acid formed on peroxiredoxins to regenerate the } \\
\text { cysteine thiol Prx }(\text { oxidized })+\operatorname{Srx}+\text { ATP }+ \text { Thiol } \rightarrow \text { Prx (reduced })+ \text { ADP } \\
+ \text { ooxidized thiol }\end{array}$ \\
\hline \multirow[t]{2}{*}{ Glutathione reductase } & Catalyzes the reduction of oxidized glutathione to its reduced form \\
\hline & $\mathrm{GSSG}+\mathrm{NADPH} \rightarrow 2 \mathrm{GSH}+\mathrm{NADP}^{+}$ \\
\hline GSTs & $\begin{array}{l}\text { GSTs catalyze the conjugation of GSH (via a sulfhydryl group) to electrophilic } \\
\text { centers on substrates to render them more soluble and to detoxify them. } \\
\text { Peroxidized lipids and xenobiotics are detoxified by GSTs }\end{array}$ \\
\hline Methionine sulfoxide reductases & Reduces oxidized methionine residues in proteins \\
\hline
\end{tabular}

ATP, adenosine triphosphate; GSH, glutathione, reduced form; GSSG, glutathione, oxidized form; GSTs, glutathione S-transferases.

predominantly cytosolic, but has also been localized to mitochondria and endosomes $(260,443)$. Nascent SOD1 is inactive in its monomeric form and is converted to its active dimeric form by the copper chaperone for superoxide dismutase (CCS), which mediates the insertion of the copper ion and an intramolecular disulfide bond $(76,115,148)$. The disulfide bridge is essential for the catalytic activity of SOD1. The copper reacts in a ping-pong mechanism where one $\mathrm{O}_{2}{ }^{\bullet-}$ molecule is reduced to $\mathrm{H}_{2} \mathrm{O}_{2}$ and a second $\mathrm{O}_{2}{ }^{\bullet-}$ is oxidized to $\mathrm{O}_{2}$. Interestingly, SOD1-deficient mice are viable and similar to wild-type mice at young ages but as they age, these mice suffer from a variety of maladies ranging from macular degeneration, oxidative stress, hepatocarcinogenesis, and infertility to muscle wasting, denervation of motor neurons, and behavioral changes (140, 221, 238, 418, 557). Mutations in SOD1 have also been linked to neurodegeneration as discussed later.

SOD2 is present exclusively in the inner mitochondrial space with the human enzyme existing as a tetramer of four identical $22 \mathrm{kDa}$ subunits $(337,532)$. SOD2 is encoded by nuclear DNA, imported into the mitochondrial matrix posttranslationally, and assembled into the active enzyme, involving the incorporation of a manganese ion in the mitochondrial matrix (540). Unlike SOD1, SOD2 does not exhibit product inhibition. In addition to scavenging $\mathrm{O}_{2}{ }^{\bullet-}$, SOD2 has been reported to inhibit $\mathrm{ONOO}^{-}$formation, itself being inactivated by excess $\mathrm{ONOO}^{-}$(407, 504). SOD2deleted mice survive up to 3 weeks of age and exhibit several abnormalities, including severe anemia, degeneration of neurons in the basal ganglia and brainstem, and progressive motor disturbances characterized by weakness, rapid fatigue, and circling behavior (295).

SOD3, the most recently discovered member of the family, is also a $\mathrm{Cu} / \mathrm{Zn} \mathrm{SOD}$, but is secreted and exists as a homotetramer of $30 \mathrm{kDa}$ subunits $(165,330)$. This extracellular enzyme was first detected in human plasma, lymph, ascites, and cerebrospinal fluids (331). It is also present in the extracellular matrix and on cell surfaces, anchored by interac- tions with the heparan sulfate proteoglycan, collagen, and fibulin-5 $(330,366,395,446)$. SOD3 is modified by N-linked glycosylation, which can be used to separate it from the other SOD enzymes $(329,509)$. Similar to SOD1, deletion of SOD3 is not lethal in mice but predisposes the mice to developing various pathologies such as sensitivity to hypoxia, hyperoxia, renal injury, and hypertension among others (72).

2. Catalases. Catalases are enzymes that degrade $\mathrm{H}_{2} \mathrm{O}_{2}$ to water and $\mathrm{O}_{2}$ with high efficiency $(10,180,406)$. Human catalase is a homotetramer of $62 \mathrm{kDa}$ monomers, each subunit containing a prosthetic heme group (406). Catalases have a peroxisome targeting signal and are predominantly present in peroxisomes, where tetramerization and heme incorporation occur $(293,405)$. Mice deleted for catalase develop normally and exhibit no gross abnormalities, but exhibited slower decomposition of extracellular $\mathrm{H}_{2} \mathrm{O}_{2}$ compared with wild-type mice and also higher susceptibility to mitochondrial dysfunction induced by traumatic brain injury (222).

3. Glutathione peroxidases (enzymes). Glutathione peroxidase (GPx) enzymes utilize GSH to reduce peroxides to produce glutathione disulfide and water (154). At least eight different isoforms of GPx exist (GPx1-8), of which GPx 1 is most abundant, present in the cytosol and mitochondria (55). Among these enzymes, GPx1-4 and GPx6 in humans are selenium-containing enzymes (298). GPx5, 7, and 8 use cysteine in place of selenium (55). Gpx1 $1^{-/-}$mice are phenotypically normal with normal life spans, but are more susceptible to oxidants, mitochondrial toxins, and ischemia/ reperfusion injury $(123,271,558)$. GPx2 is enriched in the gastrointestinal tract and GPx3 in the kidneys (143, 156, 374). Similar to $G p x 1^{-/-}$mice, $G p x 2^{-/-}$and $G p x 3^{-/-}$do not have gross morphological abnormalities, but exhibit increased sensitivity to stress $(155,248)$. Of these isoforms, GPx4 has several unique features (105). GPx4, a monomeric enzyme, exists as three isoforms through alternative splicing 
and transcription initiation: cytosolic GPx4, mitochondrial GPx4 (mGPx4), and nuclear GPx4 (nGPx4). Cytosolic GPx4 is essential for embryonic development and cell survival as systemic Gpx4 caused lethality, while nGPx4 and mGPx4 play roles in male fertility and spermatogenesis $(456,551)$. Besides acting on hydroperoxides and lipid hydroperoxides, substrates for all glutathione peroxidases, GPx4 has the unique ability of reducing phospholipid-associated hydroperoxides in biological membranes to the corresponding alcohols. This enzyme can also utilize thiols other than GSH as a reductant. GPx4 is abundant in the brain and is present in both glial cells and neurons and protects them against oxidative damage $(398,563,567)$. Among the GPx proteins, GPx 7 and GPx 8 are ER-localized protein disulfide isomerase (PDI) peroxidases, functioning as stress sensors and transducers (367). GPx7 binds to its target proteins such as PDI and $78 \mathrm{kDa}$ glucose-regulated protein, also known as immunoglobulin heavy chain binding protein, and modulates disulfide bond formation in response to stress stimuli (89).

4. Peroxiredoxins (enzymes). The other large class of enzymes that reduce peroxides are peroxiredoxins (Prxs), which utilize cysteine, often called the "peroxidatic" cysteine, $\mathrm{C}_{\mathrm{p}}$, the site of oxidation by peroxides $(422,423)$. Oxidation of this residue $\left(\mathrm{C}_{\mathrm{P}}-\mathrm{SH}\right)$ generates cysteine sulfenic acid $\left(\mathrm{C}_{\mathrm{P}}-\mathrm{SOH}\right)$, which then reacts with another cysteine, termed the resolving cysteine, $\mathrm{C}_{\mathrm{R}}$, to form a disulfide, which can then be reduced by an electron donor. The $\mathrm{C}_{\mathrm{p}}$ residue can be oxidized by $\mathrm{H}_{2} \mathrm{O}_{2}$, lipid peroxide, or $\mathrm{ONOO}^{-}$very rapidly, with rate constants $1 \times 10^{6}-10^{8} M^{-1} \cdot \mathrm{s}^{-1}$, which are 5-7 orders of magnitude higher than those for small thiols (538). Depending on the location of the resolving cysteine, Prx enzymes have been classified into 2-Cys, atypical 2-Cys, and 1-Cys Prx subfamilies. Prx enzymes are homodimeric and contain two conserved $\left(C_{P}\right.$ and $\left.C_{R}\right)$ cysteine residues per subunit, where intersubunit disulfides are formed. Atypical 2Cys Prx enzymes, on the contrary, form intramolecular disulfide bonds. 1-Cys Prx, as the name indicates, has only one cysteine participating in the reaction. The $C_{P}$ here forms a disulfide with $\mathrm{C}_{\mathrm{R}}-\mathrm{SH}$ of other proteins or thiols (151). Mammals possess six Prx isoforms of which PrxI to PrxIV are 2-Cys (typical) Prx enzymes. PrxV is a typical 2-Cys and PrxVI a 1-Cys enzyme. The disulfide bond formed during detoxification of peroxides can be reduced by the antioxidant enzyme sulfiredoxin to restore peroxidase activity $(47,541)$. More recently, Prx enzymes have been reported to participate in a relay system, which leads to oxidation of target proteins (as opposed to reduction) to facilitate ROS signaling, suggesting additional roles as sensors and transmitters of $\mathrm{H}_{2} \mathrm{O}_{2}$ signals $(488,489)$.

In addition to these direct acting enzymes, several other proteins act to maintain redox homeostasis in cells some of which reverse the effects of free radicals (Table 2). One of these types of repair enzymes are the methionine sulfoxide reductases (MSRs: MsrA and B), which convert oxidized methionine residues (MetO) on proteins to their original state $(356,483)$. MSRs use thiol/selenothiol chemistry to reduce oxidized methionines using the reducing power generally provided by the thioredoxin system (506). Apart from these repair proteins, others regulate the redox mileu in cells by synthesizing antioxidants or by eliminating the toxic metabolites. For example, glutathione S-transferase can help eliminate $4 \mathrm{HNE}$, a toxic metabolite that accumulates during lipid peroxidation $(11,24)$. Similarly, biosynthetic enzymes of antioxidants such as CSE, the enzyme that produces cysteine, can be upregulated by cells in response to stress (382, $384,453,454)$. Thus, cells have evolved multiple mechanisms to maintain redox signaling during different conditions and stages of development.

\section{Dysregulation of Redox Homeostasis in Neurodegeneration}

Several neurodegenerative disorders are associated with elevated oxidative stress. Given the fact that the brain is one of the most metabolically active tissues, it is not surprising that the brain is also highly susceptible to oxidative damage. Some of the antioxidant enzymes such as catalase are present in lower levels in the brain $(202,204)$. The brain is also rich in transition metals such as iron, which can inflict damage via the generation of $\bullet \mathrm{OH}$ radicals. Consistent with these findings, in the stroke-prone spontaneously hypertensive rats, levels of oxidized proteins were significantly increased in the brain, but not kidneys or serum (347). However, systematic studies of oxidative damage in various organs in comparison with the brain are still lacking. Generation of free radicals is intimately linked to metabolism so that disruption of specific physiological processes can elicit neuronal death. It has also been observed that selective vulnerability of specific regions in the brain occurs in different neurodegenerative diseases (529) (Fig. 4). A common feature of these disorders is that redox imbalance involves dysregulation at multiple levels $v i z$, increased production of free radicals and damaging species, malfunctioning antioxidant defense mechanisms, and repair pathways. Here we discuss redox disturbances in a monogenic disorder, HD, as an example, in which several features pertaining to redox signaling are also encountered in other neurodegenerative disorders, including AD, PD, ALS, ataxias, and autism.

\section{A. Huntington's disease}

$\mathrm{HD}$ is an autosomal dominant neurodegenerative disease characterized by expansion of polyglutamine repeats in the protein huntingtin (1). Mutant huntingtin aggregates and causes widespread damage ranging from transcriptional dysregulation to motor and cognitive deficits. Greater than 40 repeats predispose an individual to the disease, which is characterized by involuntary chorea and cachexia during disease progression. Presently, there is no cure for the disease, although several drugs that mitigate symptoms are available. The various drugs used to treat symptoms of HD range from antidepressants to antipsychotics and those used to treat chorea (517). The food and drug administration (FDA) has approved the use of tetrabenezine for treatment of chorea. Although tetrabenezine effectively reduces chorea in HD patients, its use has been associated with side effects such as suicidal thoughts, depression, and restlessness $(232,294)$. HD primarily affects the corpus striatum of the brain, which regulates motor functions (Fig. 4). The medium spiny neurons in the striatum degenerate, leading to striatal shrinkage and atrophy. The cerebral cortex is also affected during the later stages of the disease. At the molecular level, several abnormalities have been reported in HD, which include 


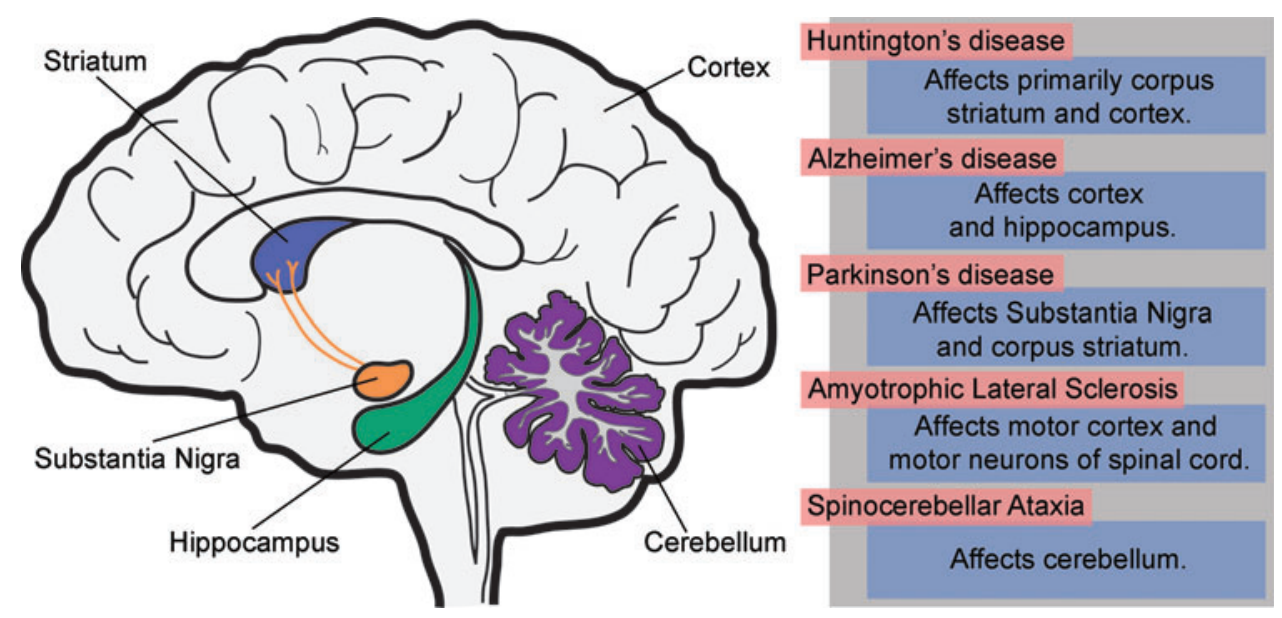

FIG. 4. Selective vulnerability of specific brain regions in various neurodegenerative diseases. Although redox imbalance is a common feature in various neurodegenerative diseases, each disease affects a specific region of the central nervous system. HD affects primarily the corpus striatum, followed by the cortex in later stages of the disease. Alzheimer's disease affects the cortex (starting with the entorhinal cortex) and the hippocampus leading to cognitive decline. The substantia nigra and the striatum are affected in PD. In ALS, which culminates in paralysis, the motor cortex of the brain and the motor neurons of the spinal cord degenerate. In SCA and several other forms of ataxia, the cerebellum is targeted. ALS, amyotrophic lateral sclerosis; HD, Huntington's disease; PD, Parkinson's disease; SCA, spinocerebellar ataxia. Color images are available online.

elevated oxidative stress in affected regions and compromised redox signaling pathways.

1. Oxidative stress in HD. Numerous studies have reported oxidative damage in HD cells and tissues (59, 85, 282, 481). Higher levels of lipid peroxidation and low GSH content have been reported in the plasma of HD patients (270). Oxidative damage to both nuclear and mtDNA is caused by mutant huntingtin with the basal ganglia being especially vulnerable (58). Postmortem caudate tissue from HD patients displays elevated 8-hydroxydeoxyguanosine (8-OHdG), a marker for oxidative DNA damage. Increases in $8-\mathrm{OHdG}$ have also been observed in serum and leukocytes of HD patients $(85,217)$. In both 3-nitropropionic acid-treated mice, a chemical model of $\mathrm{HD}$, and the R6/2 model of HD, quantitative PCR reveals extensive mtDNA damage (7). In mouse embryonic cells derived from YAC128 $\mathrm{HD}$ mice, increased $\mathrm{O}_{2}{ }^{\bullet-}$ production and dysregulated calcium signaling cause elevated oxidative stress compared with wild-type cells. These findings were recapitulated in YAC128 HD striatal medium spiny neurons. Similar results were observed in fibroblasts derived from HD patients and a mouse model of HD (525).

2. Compromised low-molecular-weight antioxidant metabolism in HD. HD has been associated with decreased levels of endogenous antioxidant molecules, which contribute to the oxidative stress linked to pathogenesis.

a. Reduced cysteine and GSH levels in HD. The metabolism of small-molecular-weight thiols such as cysteine and GSH is compromised in $\mathrm{HD}(381,383,453)$. We have shown previously that depletion of CSE, the biosynthetic enzyme for cysteine and the gaseous signaling molecule, hydrogen sulfide, contributes to neurotoxicity in HD. The transcription factor for basal expression of CSE, specificity protein 1 (SP1), is sequestered by mutant huntingtin, which reduces
CSE expression in HD $(137,381)$. Low levels of CSE were observed in striatal cell culture models of HD, mouse models, and in postmortem striatal tissue from HD patients. The depletion is specific to the striatum and the degree of decrease correlates with the severity of the disease. The cortex exhibits a decline in CSE but during later stages of the disease. CSE is a key enzyme in the reverse transsulfuration pathway, in which cysteine is synthesized by transfer of a sulfur atom from homocysteine, which in turn is derived from dietary methionine (Fig. 5). The reverse transsulfuration pathway plays a central role in redox-regulated signaling nodes in cells. The decrease in CSE is accompanied by a reduction in cysteine and $\mathrm{H}_{2} \mathrm{~S}$ production $(381,454)$. In addition to its biosynthesis, the transport of cysteine or its oxidized form of cystine is compromised in HD $(161,303)$. During conditions of stress, such as amino acid deprivation or ER stress, expression of CSE is dependent on the activating transcription factor 4 (ATF4). ATF4 is a stress-inducible protein that regulates the expression of amino acid biosynthetic and transport genes during conditions of amino acid deprivation, ER stress, as well as other stimuli $(453,454)$. The induction of ATF4 in response to cysteine deprivation is blunted in striatal progenitor cells harboring mutant huntingtin (453). Interestingly, the muted response of ATF4 in HD striatal cells occurs selectively for cysteine deprivation and not in response to deprivation of other amino acids or other forms of stress, indicating that specific properties of cysteine are compromised in HD. The abnormality stems from the chronic oxidative stress occurring in HD due to depletion of CSE leading to a vicious cycle of oxidative stress and impaired response of restorative pathways leading to further damage and ultimately cell death (453) (Fig. 6). Cysteine is a component of GSH, and the availability of cysteine is the ratelimiting step for GSH production. In addition, cysteine is a potent antioxidant on its own; therefore, its scarcity is associated with redox imbalance. Depletion of CSE and cysteine 


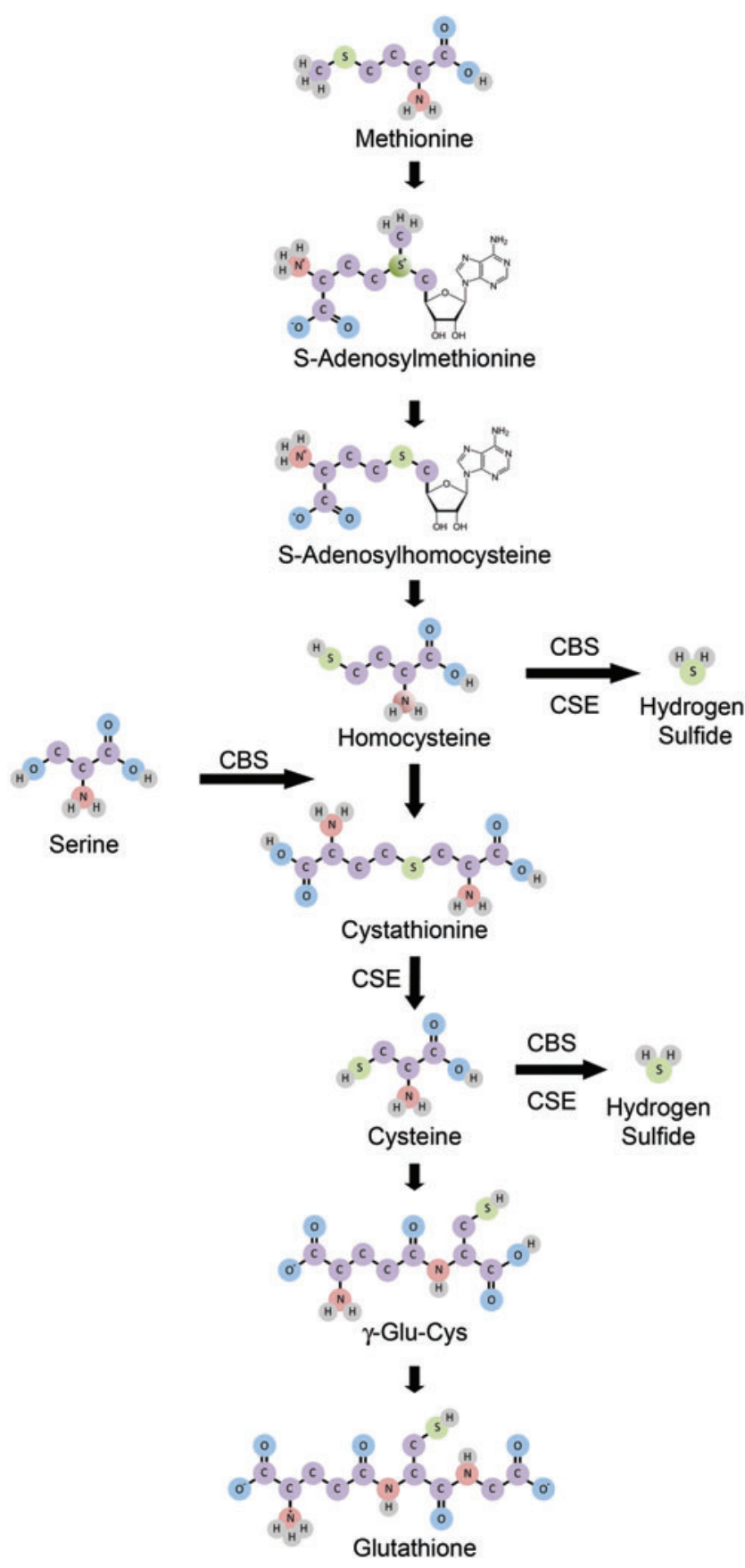

FIG. 5. The reverse transsulfuration pathway. The reverse transsulfuration pathway is responsible for the transfer of sulfur from homocysteine, which in turn is derived from dietary methionine, to cysteine. Cysteine is synthesized from homocysteine by the action of CBS and CSE. CBS condenses serine and homocysteine to form cystathionine, which is acted on by CSE to produce cysteine and the gaseous signaling molecule, $\mathrm{H}_{2} \mathrm{~S}$. Both CBS and CSE can generate $\mathrm{H}_{2} \mathrm{~S}$ from homocysteine. Cysteine can also be channeled into glutathione biosynthesis to maintain redox balance in cells. The availability of cysteine is the rate-limiting step for the synthesis of glutathione. CBS, cystathionine $\beta$-synthase; CSE, cystathionine $\gamma$-lyase; $\mathrm{H}_{2} \mathrm{~S}$, hydrogen sulfide. Color images are available online. results in elevated levels of protein carbonylation, protein nitration, and lipid peroxidation in mouse models of HD (381, 453). Cysteine is also the precursor for several cytoprotective molecules such as $\mathrm{H}_{2} \mathrm{~S}$, lanthionine, taurine, and glutathione (Fig. 7). Of these, $\mathrm{H}_{2} \mathrm{~S}$ plays pleiotropic roles in cell physiology, modulating signaling pathways via sulfhydration/persulfidation of reactive cysteine residues on target proteins $(360,382,384$ 386). Thus, disrupted cysteine homeostasis has multifaceted consequences. Accordingly, supplementation of cysteine in the diet and N-acetylcysteine (NAC) in the drinking water of the R6/2 mouse model of HD ameliorates symptoms and increases life span (381). These studies also showed that mice on a high cysteine diet exhibited improved motor function and decreased brain pathology. Independent studies utilizing NAC report beneficial effects on behavioral aspects of HD (542).

b. Reduced ascorbate uptake in HD. Ascorbic acid or vitamin $\mathrm{C}$ is an antioxidant that is highly enriched in the brain, where it can regulate neuronal metabolism $(80,477)$. Ascorbate defends neurons against oxidative damage and modulates neuronal metabolism during synaptic activity (110). Ascorbate mediates lactate utilization as an alternative neuronal energy substrate (79). During synaptic activity, the ascorbate released prevents neuronal glucose utilization and stimulates lactate utilization to sustain brain activity. Ascorbate, thus, acts as a switch to regulate use of other substrates such as lactate for energy production via a GLUT3 (glucose transporter 3)-dependent mechanism (38). High-affinity, sodium-dependent vitamin $\mathrm{C}$ transporters (SVCTs) transport vitamin C into neurons (78). Striatal cells derived from HdhQ111 mouse model of HD have impaired trafficking of SVCT2 to the membrane leading to lowered ascorbate transport and altered antioxidant and bioenergetic capacity (8). Flux of ascorbate from astrocytes to neuronal cells in brain slices of R6/2 mouse model of HD is impaired and the ascorbate metabolic switch is affected in HD. Synaptic activity generates oxidants that oxidize ascorbate to dehydroascorbate, which is released by neurons and taken up by astrocytes to regenerate ascorbate via GSH-dependent processes $(109,275)$. In presymptomatic stages of $\mathrm{HD}$, astrocytes do not release sufficient ascorbate to be utilized by neurons. Interestingly, HD is also associated with lowered surface expression of GLUT3, which disrupts utilization of lactate as an energy substrate (167). It was proposed that the increased oxidative stress associated with HD would utilize ascorbate and minimize its availability to regulate energetics (110).

3. Elevated production of free radicals in HD. Several factors contribute to the elevated levels of free radicals in HD. A few of these pathways are given below.

a. Activation of NOX enzymes. Increased activity of NOX has been observed in HD and may contribute to neurotoxicity. NOX catalyzes the formation of $\mathrm{O}_{2}{ }^{\bullet-}$ from oxygen, which damages cellular components as described earlier. Synaptosome fractions derived from the cortex and striatum of HD $(140 \mathrm{Q} / 140 \mathrm{Q})$ mice display elevated NOX activity with the striatum exhibiting the greatest increase. Treatment with NOX inhibitors such as diphenyleneiodonium, apocynin, and VAS2870 attenuates cell death and toxicity (516). 

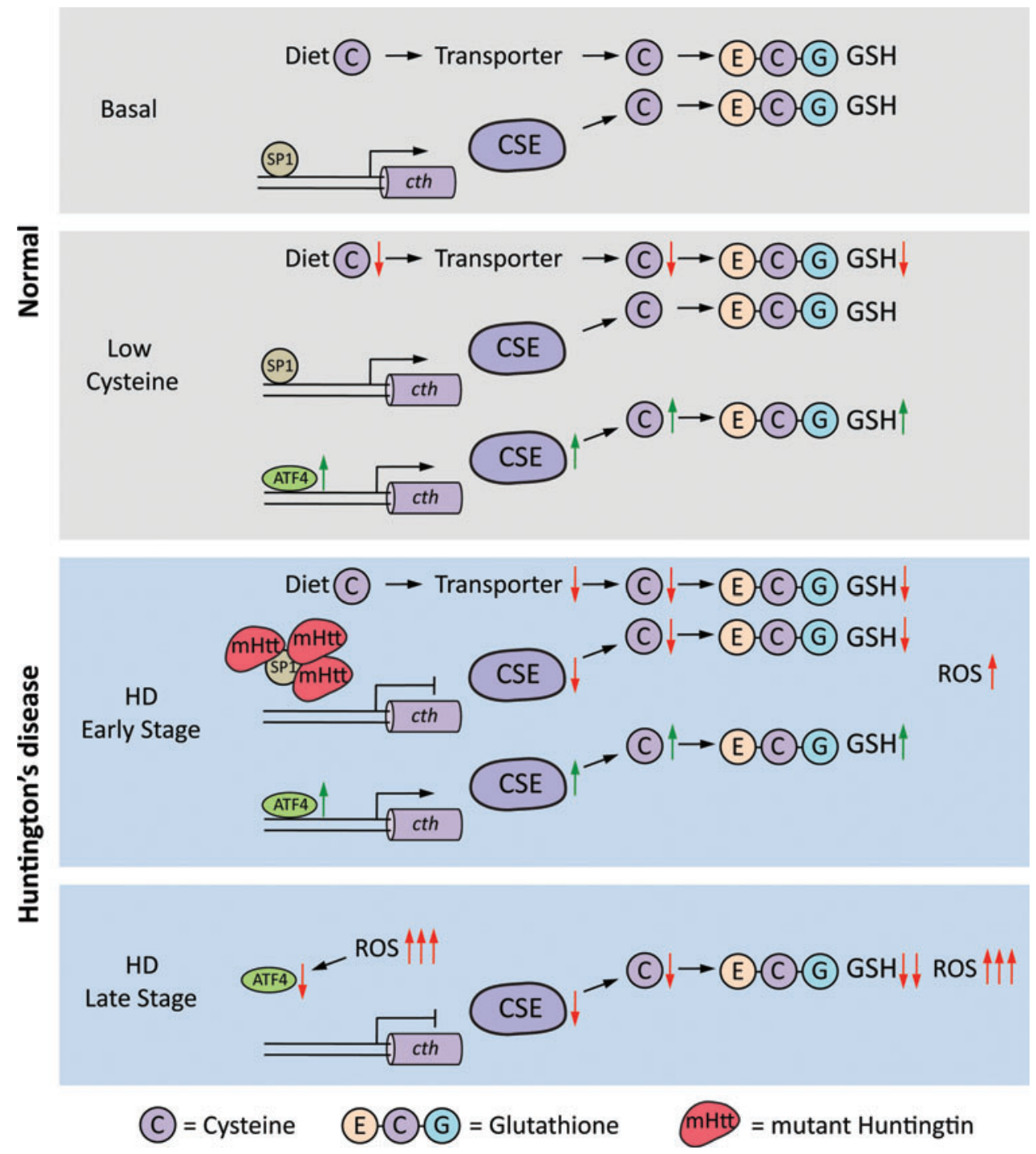

FIG. 6. Impaired cysteine metabolism in HD. In normal cells (shown in gray background) under basal conditions, cysteine, denoted as C, is taken up from the diet via its transporters or synthesized CSE, its biosynthetic enzyme for cysteine. Basal expression CSE is regulated by the transcription factor SP1. The availability of cysteine is the rate-limiting step for the generation of glutathione (GSH), a tripeptide of glutamate (E), cysteine (C), and glycine (G). During conditions of low cysteine, ATF4 is induced and regulates transcription of CSE. In HD cells (shown in blue panels), CSE is depleted due to sequestration of SP1 by mutant huntingtin (mHtt), leading to low cysteine and increased ROS levels. The deficiency of CSE can be compensated by ATF4 induction in initial stages of HD. However, elevated oxidative stress, in later stages of $\mathrm{HD}$, compromises the function of the transcription factor, ATF4, which prevents induction of CSE, further increasing oxidative damage and resulting in a vicious feedforward cycle, where low levels of CSE and cysteine cause increased oxidative damage such that protective responses to stress decline, leading to neurodegeneration. ATF4, activating transcription factor 4; SP1, specificity protein 1. Color images are available online.

b. Redox metals and HD. Dysregulated iron metabolism is a common feature of several neurodegenerative disorders (263, 438). Excess iron deposition has been reported in the basal ganglia of the brain in HD (30). Furthermore, neonatal iron supplementation in the YAC128 mouse model of HD accelerates disease progression (43). Copper has also been linked to disease progression in HD. Elevated copper deposition increases oxidative stress and aggregation of mutant huntingtin, which can be reversed by copper chelators $(160,546)$.

c. Defective DNA repair in response to oxidative stress. Nuclear DNA damage occurs in HD. The ataxia-telangiectasia mutated (ATM) DNA repair cascade is affected, and base excision repair regulated by the ATM protein in response to oxidative stress is defective (316). Huntingtin acts as a scaffolding protein in the ATM repair complex (323). Oxidative stress mediates CAG expansion in HD fibroblasts. The expansion arises during the process of removal of oxidized DNA bases (8-oxo-G lesions), and is dependent on the DNA glycosylase, OGG1 (278). A "toxic oxidation cycle" model was proposed in which somatic mutations mediate onset and progression of the disease. As oxidative lesions in the brain accumulate with age, a DNA damage response involving OGG1 repair through a single-stranded break mechanism is mounted. 


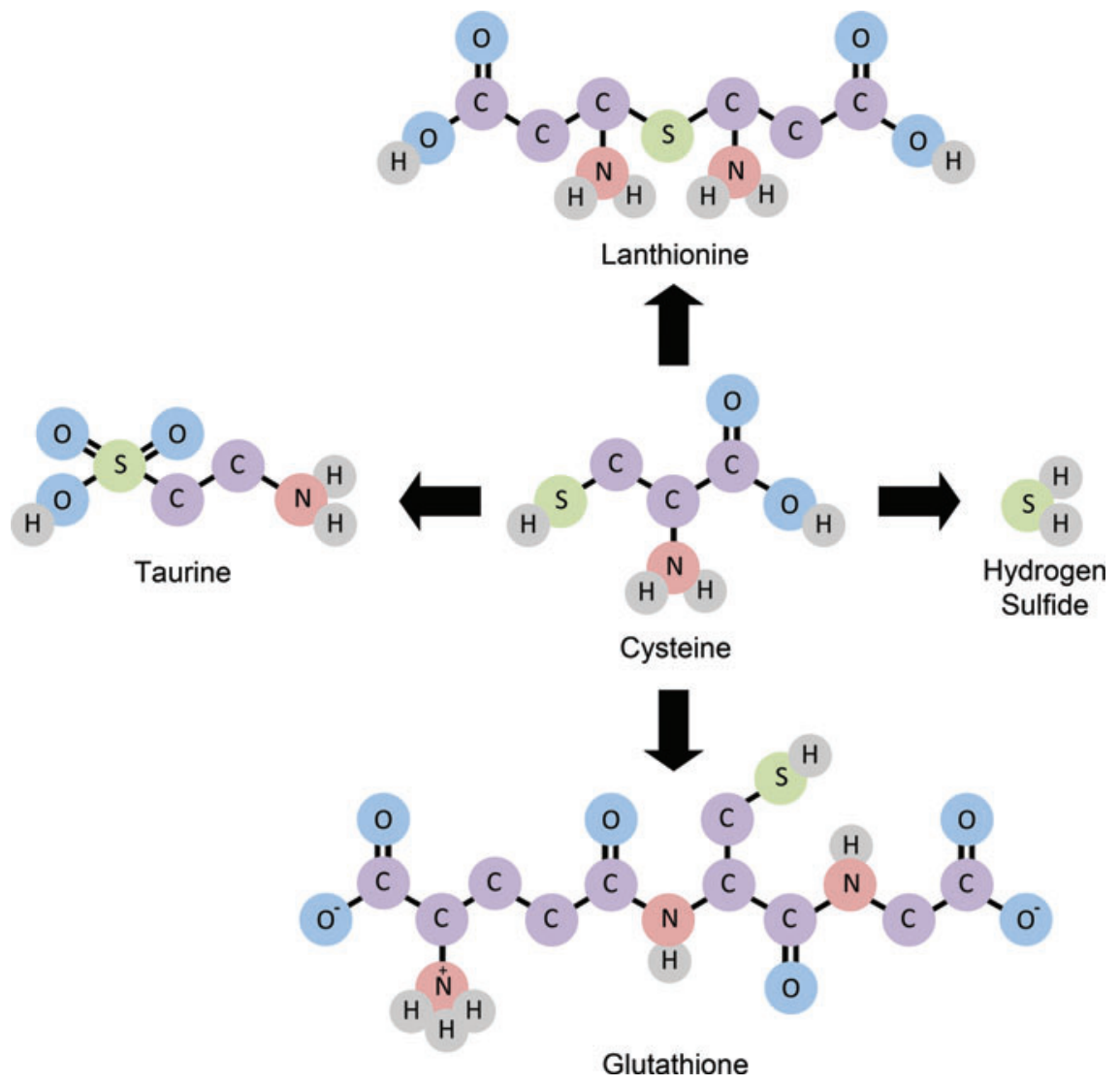

FIG. 7. Sulfur containing endogenous molecules derived from cysteine. Cysteine serves as a precursor of several sulfur containing molecules, which include lanthionine, taurine, and $\mathrm{H}_{2} \mathrm{~S}$. All these sulfur containing metabolites have neuroprotective roles. $\mathrm{H}_{2} \mathrm{~S}$ plays central roles in various physiological processes ranging from mitigating inflammation to clearance of misfolded proteins and maintenance of redox homeostasis. Color images are available online.
The repair is error prone and thus leads to expansion of the CAG repeats, which further exacerbates the cycle.

d. Excessive NMDAR stimulation. Overactivation of the NMDA type of glutamate receptors is a feature of HD. Excessive stimulation of NMDARs causes elevated oxidative and nitrosative stress $(136,362)$. Neurons from the subthalamic nuclei in the BACHD model of HD exhibit elevated oxidative stress, which is decreased by treatment with NMDAR antagonists (16).

e. Impaired antioxidant response pathways in HD. The nuclear factor erythroid 2-related factor 2 (Nrf2) is a master transcriptional regulator of the cellular antioxidant stress response and detoxification pathways. It is a basic leucine zipper protein that cooperates with other proteins and activates genes with an antioxidant response element/electrophile response element or ARE/EpRE (213, 224, 440, 497). Under basal conditions, Nrf2 is sequestered in the cytoplasm by kelch-like ECH-associated protein 1-Cul3 E3 ligase complex, which ubiquitinates it, targeting it for degradation by the proteasome system $(114,240,273,562)$. The Nrf2 pathway is dysregulated in HD, which could increase free radical levels and cause mitochondrial dysfunction (249). Homologous to the E6-AP carboxyl terminus domain and ankyrin repeat containing E3 ubiquitin-protein ligase 1 , an activator of $\mathrm{Nrf} 2$, is downregulated in $\mathrm{HD}$ leading to suboptimal responses to oxidative stress (435).

f. Mitochondrial dysfunction in HD. The involvement of mitochondrial dysfunction in HD was first suggested by nuclear magnetic resonance spectroscopy, which revealed ele- vated lactate levels in the cortex and striatum of HD patients, which correlated with the CAG repeat length $(247,276)$. Biochemical studies also confirmed the reduced activity of mitochondrial complex II, III, and IV in postmortem HD caudate putamen and cerebral cortex $(54,58,189)$. Lymphocytes derived from HD patients also exhibit defective mitochondrial bioenergetics, which are dependent on the CAG repeat length (467). In addition, 3-nitropropionic acid, a mitochondrial complex II inhibitor, induces HD-like symptoms in mice (57). In HD, two major components of mitochondrial complex II, the 30-kDa iron/sulfur (Ip) subunit and the $70-\mathrm{kDa} F A D(\mathrm{Fp})$ subunit, are preferentially decreased in the striatum of HD (40). Decreases in SDH activity have deleterious consequences, as it is a component of both the ETC and the Krebs cycle and would affect both energy-producing pathways. Deficits in complex II play pivotal roles in mediating neurodegeneration, and overexpression of complex II ameliorates disease progression (118). Mitochondrial deficits in HD are also caused by dysregulation of transcription factors and their coactivators that modulate mitochondrial function. Peroxisome proliferator-activated receptor gamma (PPAR $\gamma)$ regulates several processes such as fatty acid oxidation, mitochondrial biogenesis, turnover, energy homeostasis, immune responses, and antioxidant defense. The peroxisome proliferator-activated receptor gamma coactivator 1 -alpha $(\mathrm{PGC} 1 \alpha)$ expression is reduced in HD leading to mitochondrial dysfunction. PGC $1 \alpha$ plays important roles in mitochondrial biogenesis, respiration, and oxidative stress response (251). Mutant huntingtin represses transcription of $\mathrm{PGC} 1 \alpha$ by cAMP response element-binding protein (CREB)/transcription initiation factor TFIID subunit 4, the transcription factors regulating PGC1 $\alpha$. Deleting PGC1 $\alpha$ in 
HD knockin mice by crossing these mice with $\mathrm{PGC} 1 \alpha$ knockout mice exacerbates degeneration of medium spiny neurons of the striatum leading to motor and behavioral deficits. Thus, restoring PGC1 $\alpha$ expression in the striatum by lentiviral injection mitigates symptoms (113). Imbalanced redox in mitochondria is a component of another vicious cycle in which free radicalinduced damage compromises basic mitochondrial functions such as respiration, leading to further oxidative stress. Thus, removal of mitochondrial damage by mitochondrial fission or mitophagy becomes necessary to curtail excessive free radical generation and damage. In HD, aberrant mitophagy has been observed in which mutant huntingtin binds to valosincontaining protein (VCP), an ATPase and component of the ubiquitin proteasome system, and causes excess mitophagy and thus neurotoxicity (194). VCP associates with its cofactor UBXdomain containing protein 1 to cause degradation of the outer mitochondrial membrane protein myeloid cell leukemia sequence 1. Mutant huntingtin overactivates this process and compromises mitochondrial integrity (193).

4. Huntingtin as a sensor of oxidative stress. Although most studies have focused globally on oxidative stress mediating cytotoxicity in HD, the role of wild-type huntingtin itself as a redox sensor has been less explored. The N17 domain, the first 17 amino acids at the amino terminus of huntingtin, determines its subcellular localization. N17 is an amphipathic alpha helix (428) and can direct nuclear export (346) and anchoring to ER membranes to target huntingtin to the cytoplasm under normal conditions (18). Post-translational modifications of the N17 domain, such as phosphorylation, acetylation, and SUMOylation, regulate huntingtin localization (190, 485, 508). Stress-dependent phosphorylation of two serine residues Ser13 and Ser16 promotes nuclear localization by preventing interaction of huntingtin with chromosome region maintenance protein 1 (CRM1) thereby preventing nuclear export (17). During oxidative stress conditions, the oxidation of a methionine residue at Met8 on the N17 domain can stimulate phosphorylation of the domain and thus nuclear localization. The response of mutant huntingtin to oxidative stress could be a slower process becoming more pronounced in later stages of disease progression where the oxidative burden is higher. While oxidation of Met8 on mutant huntingtin also triggers its nuclear translocation, export from the nucleus is hampered due to the aberrant interaction with CRM1 resulting in nuclear accumulation and pathology. An unanswered question is why huntingtin translocates to the nucleus in response to oxidative stress. Wild-type huntingtin has been shown to have roles in the nucleus, such as stimulation of transcription of brainderived neurotrophic factor (573). Thus, it is possible that huntingtin could be involved in transcriptional regulation of stress response genes.

\section{B. Alzheimer's disease}

$\mathrm{AD}$ is the most prevalent neurodegenerative disease reported till date. Symptoms of AD include dementia, impaired spatial memory, and other cognitive deficits. The disease has multifactorial origins unlike HD, which is caused by a single mutation in a single gene and therefore monogenic. AD can arise due to sporadic or familial causes. The region of the brain first affected in AD is the entorhinal cortex. The disease predominantly affects the cerebral cortex and the hippo- campus (Fig. 4). Aggregation of $\beta$-amyloid $(\mathrm{A} \beta)$ and tau proteins is the hallmark of the disease, causing deposition of amyloid plaques and neurofibrillary tangles, respectively. The amyloid precursor protein (APP) is the precursor of $\mathrm{A} \beta$ peptides. APP can undergo cleavage at different sites by two major pathways: the nonamyloidogenic pathway and the amyloidogenic pathway (Fig. 8). Tau aggregation, on the contrary, is linked to its hyperphosphorylation (336). Elevated oxidative stress has also been associated with $\mathrm{AD}$, similar to several neurodegenerative diseases $(60,491)$. Increased levels of lipid peroxidation, protein carbonylation, and protein nitration have been reported in $\mathrm{AD}$. As in $\mathrm{HD}$, elevated DNA damage has also been associated with $\mathrm{AD}$ (52).

1. Amyloid beta, tau, and oxidative stress. A $\beta(1-42)$, generated by cleavage of APP, by enzymes such as $\beta$ secretase 1 (BACE1), induces oxidative stress (Fig. 8). A $\beta$ has been shown to reduce metal ions such as $\mathrm{Fe}^{3+}$ and $\mathrm{Cu}^{2+}$ to $\mathrm{Fe}^{2+}$ and $\mathrm{Cu}^{1+}$, which can generate $\cdot \mathrm{OH}$ radicals by Fenton chemistry $(229,230)$. The levels of oxidative damage have been positively correlated to expression of BACE1 (49). $\mathrm{BACE} 1$, a transmembrane aspartyl-protease, is the major $\beta$ secretase, which cleaves APP to generate the toxic A $\beta(1-42)$ fragment (521). Deletion of BACE1 prevents APP processing to generate $\mathrm{A} \beta$ both in mice and cell culture $(64,319,426)$. Formation of $\mathrm{A} \beta$ leads to activation of the Jun N-terminal kinase (JNK) pathway, which has been implicated in upregulation of BACE1 $(191,552)$. Thus, $\mathrm{A} \beta$ and BACE1 are components of a toxic feedforward cycle where increased oxidative stress promotes BACE1 production, which further increases $\mathrm{A} \beta$ production leading to oxidative stress and further BACE1 activity (Fig. 8). Increased levels of activated JNK have been reported in postmortem AD samples (570). Thus, JNK signaling constitutes a therapeutic target for AD (553). Soluble A $\beta$ can impair cysteine and GSH disposition in cells by inhibiting the excitatory amino acid transporter 3 (EAAT3), the neuronal cysteine transporter (223). EAAT3 plays a critical role in regulating redox balance in neurons, and its depletion can promote elevated oxidative stress and age-dependent neurodegeneration (15). This component of redox imbalance can feed into the toxic cycle described earlier.

Similarly, accumulation of hyperphosphorylated tau has been reported to cause oxidative stress, and ROS have been shown to stimulate tau hyperphosphorylation $(490,569)$. Tau is the major constituent of neurofibrillary tangles and higher order structures generated by formation of disulfide bridges via cysteine residues (441). Interplay between tau and amyloid beta peptides has been reported. Amyloid beta peptides cause aggregation of tau to promote neuronal dysfunction. A variety of $\mathrm{A} \beta$ peptides are generated by cleavage of APP, which include $\mathrm{A} \beta 40$ and $\mathrm{A} \beta 42$. It was shown recently that $\mathrm{A} \beta^{*} 56$, a 56$\mathrm{kDa}$ oligomer that accumulates before early symptoms of AD manifest, alters neuronal signaling by activating CamKII, a kinase that phosphorylates tau (13). In addition to these changes, the metal content of AD brains is also higher with the concentration of iron in amyloid plaques almost twice as that of neighboring tissues, while copper and zinc content are threefold higher, which mediate oxidative stress (412).

2. Mitochondrial dysfunction. Similar to HD, mitochondrial dysfunction has been reported in AD. Sporadic mtDNA deletions up to $9 \mathrm{~kb}$ long with a commonly occurring specific 


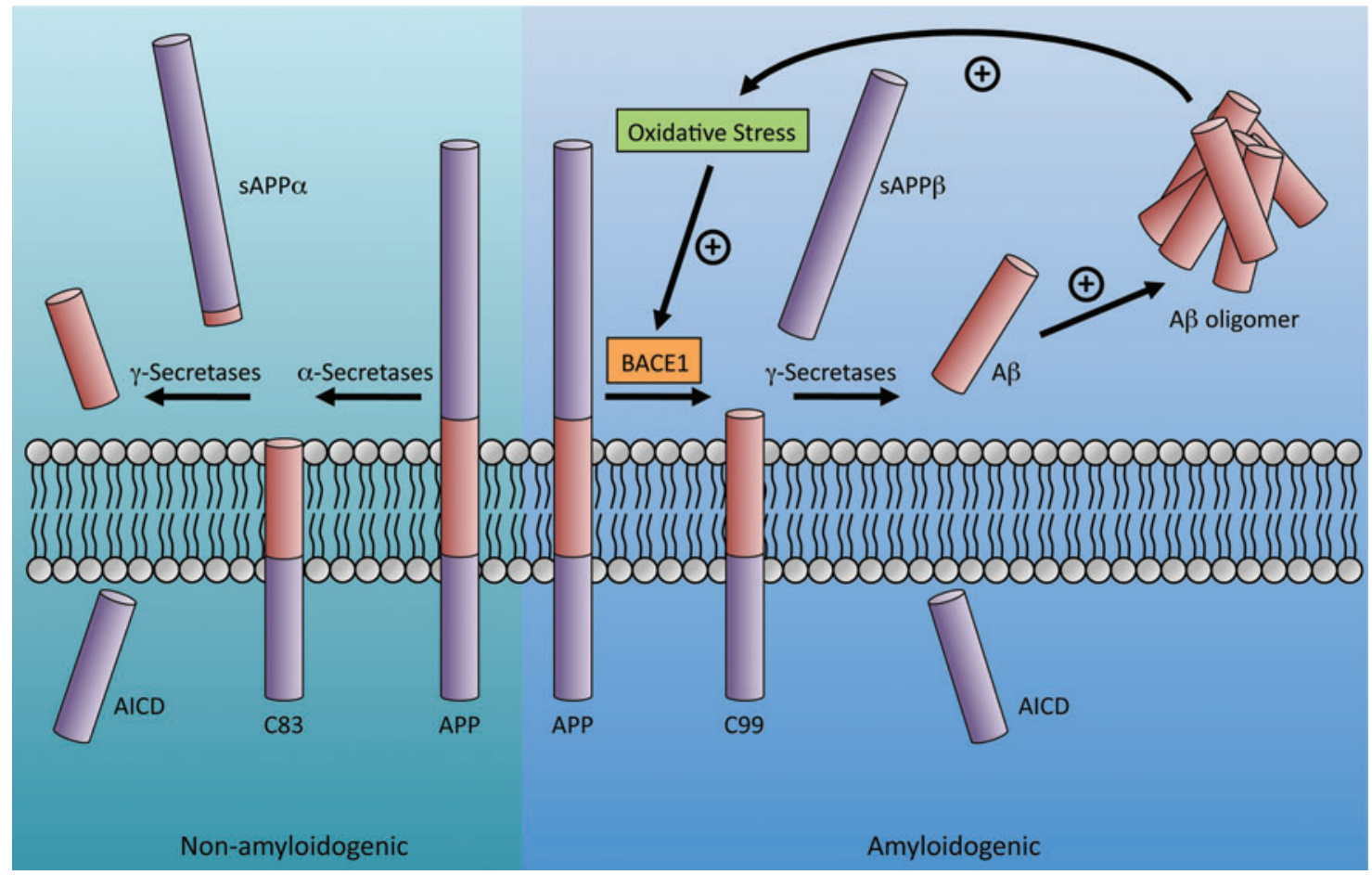

FIG. 8. Oxidative stress, BACE1, and amyloid beta aggregation. In Alzheimer's disease, there is an increased production of amyloid beta $42, \mathrm{~A} \beta(1-42)$, which is generated by the action of BACE1 on the APP. A $\beta(1-42)$ induces oxidative stress, one of the modes being reduction of metal ions such as the ferric ion to ferrous ion, which can generate hydroxyl radicals via the Fenton chemistry. A $\beta(1-42)$ production leads to the activation of the JNK pathway, which results in upregulation of BACE1, which further increases production of $\mathrm{A} \beta(1-42)$ and oxidative stress to create a cycle of oxidative stress, $\mathrm{A} \beta(1-42)$ production and aggregation leading to neurodegeneration. $\mathrm{A} \beta, \beta$-amyloid; APP, amyloid precursor protein; BACE1, $\beta$-secretase 1 ; JNK, Jun N-terminal kinase. Color images are available online.

5-kb deletion have been attributed to oxidative damage (554). The mtDNA of cortical neurons in AD patients $<75$ years of age has 15 times more of the $5 \mathrm{~kb}$ mtDNA deletion mutations than age-matched controls (107).

3. Transcriptional dysregulation. In $\mathrm{AD}$, the repressor element 1-silencing transcription factor (REST), also known as neuron-restrictive silencer factor, is depleted causing oxidative stress and suboptimal stress responses (315). REST is a repressor with functions during embryonic development of neuronal genes and becomes downregulated once terminal neuronal differentiation has occurred. REST is also induced during aging and regulates genes that mediate cell death and stress resistance. Some of the cell death proteins regulated by REST include the p38 MAP kinase (MAPK11), BAX, BID, and PUMA (315). Nuclear REST levels are diminished in neurons of affected regions in $\mathrm{AD}$, including prefrontal cortical neurons as well as the hippocampus. Neurons lacking nuclear REST are vulnerable to oxidative stress and $\mathrm{A} \beta$ toxicity and display elevated levels of apoptosis inducing genes. In cell culture, knockdown of REST results in elevated oxidative stress, which is rescued by REST overexpression or by treatment with the antioxidant NAC (315).

4. Aberrant nitrosylation. Nitrosylation is a posttranslational modification elicited by the gaseous signaling molecule ${ }^{\circ} \mathrm{NO}$ on reactive cysteine residues (484). Ni- trosylation modulates several physiologic processes in cells and influences protein activity, protein/protein interactions, and localization. Low levels of ${ }^{\bullet} \mathrm{NO}$ are usually beneficial to cells, but higher concentrations can elicit cytotoxicity. In the brain, neuronal nitric oxide synthase (nNOS) is the predominant enzyme that generates ${ }^{\circ} \mathrm{NO}$, although it is also formed by inducible nitric oxide synthase (iNOS) under conditions of stress. Excess production of ${ }^{\bullet} \mathrm{NO}$ can cause protein misfolding. For example, PDI is nitrosylated in brains of $\mathrm{AD}$ and $\mathrm{PD}$ patients, which causes improper folding of toxic proteins (512). PDI is a chaperone enzyme present in the ER that modulates formation of disulfide bonds during their synthesis and maturation $(162,186)$. In neurodegenerative disorders and during ischemia, accumulation of denatured proteins causes ER dysfunction. Under such conditions, PDI induction occurs as an adaptive response, which is compromised by nitrosylation $(226,502)$. Cell death induced by the ER stressors, thapsigargin and tunicamycin, in the cell line SH-SY5Y was largely prevented by wild-type PDI, but this protection was abrogated in the presence of an ${ }^{\bullet} \mathrm{NO}$ donor. ${ }^{\bullet} \mathrm{NO}-$ mediated nitrosylation of PDI inhibits its catalytic activity, causing accumulation of polyubiquitinated proteins and activating the unfolded protein response (512). In accordance with these findings, in the brains of $\mathrm{AD}$ and $\mathrm{PD}$ patients, accumulation of nitrosylated PDI was observed (512). Nitrosylation also disrupts metabolic processes such as glycolysis and antioxidant defense in cells. In addition, 
$\mathrm{ONOO}^{-}$generated from ${ }^{\circ} \mathrm{NO}$ mediates protein nitration. A well-characterized mode of ${ }^{\circ} \mathrm{NO}$ action in the brain is the activation of NMDARs. The neurotransmitter glutamate stimulates NMDARs and causes $\mathrm{Ca}^{2+}$ influx, which activates nNOS to generate ${ }^{\circ} \mathrm{NO}$ (501). The outcome depends on whether the NMDAR is synaptic or extrasynaptic. Under normal conditions, activation of synaptic NMDARs results in production of physiological levels of ${ }^{\bullet} \mathrm{NO}$, required to promote neuronal differentiation and survival as well as normal synaptic plasticity (317). ${ }^{\bullet} \mathrm{NO}$ activates the CREB pathway to long-term potentiation. In contrast, excess stimulation of "extrasynaptic" NMDARs results in elevated levels of ${ }^{\circ} \mathrm{NO}$ and free radicals, which contribute to synaptic dysfunction by nitrosylating dynamin-related protein 1 (Drp1) and cyclindependent kinase 5 (Cdk5) to mediate neurodegeneration (350). Dynamin-1-like protein is a GTPase that regulates mitochondrial fission. The S-nitrosylation of Drp1 causes dimer formation and increased GTPase activity, thus accelerating the process of mitochondrial fragmentation and contributing to neuronal synaptic damage or cell death (93). Cdk5 is a cyclin-dependent, neuron-specific kinase, which has roles in cell survival, axon guidance, neuronal migration, and regulation of synaptic spine density. S-nitrosylation of Cdk5 enhances its kinase activity leading to hyperphosphorylation of its substrate tau, in AD (32). In addition, SNO-Cdk5 can transfer the NO group by transnitrosylation to Drp1, its substrate (forming SNO-Drp1), in this manner possibly mediating the synaptic damage. Soluble A $\beta$ extensively demonstrated to preferentially activate extrasynaptic GluN2B (NR2B)-containing NMDARs $(265,302,498)$. Thus, targeting the extrasynaptic receptors has therapeutic benefits. The FDA-approved drug, memantine, preferentially blocks extrasynaptic oversynaptic NMDARs (545) and delays symptoms in $\mathrm{AD}$.

\section{Parkinson's disease}

PD was first described over 200 years ago as a shaking palsy and was considered to be a disease, which affects the substantia nigra pars compacta (SNpc) and striatum causing motor deficits (Fig. 4) (399). It is the second most prevalent neurodegenerative disease and affects $2-3 \%$ of aging populations older than 65 . The incidence of the disease is 5 to $>35$ cases per 100,000 individuals (511). PD is associated with loss of dopaminergic neurons in the SNpc, leading to depletion of the neurotransmitter dopamine (DA). With advancements in PD research, it is now clear that the disease affects not only the motor functions but other physiological processes such as cognition, sleep, and smell as well. Similar to $\mathrm{AD}, \mathrm{PD}$ can also arise due to genetic causes as well as occurring sporadically, with the sporadic forms comprising the vast majority of cases reported. Familial or heritable forms of PD constitute only 15\% of the cases, of which 5$10 \%$ of PD patients have monogenic forms of the disease, exhibiting a classical Mendelian type of inheritance (127, 399). Several genes have been linked to developing PD. $S N C A$, encoding $\alpha$-synuclein, was the first gene definitely associated with familial PD $(400,401)$. In addition, mutations in leucine-rich repeat kinase 2 (LRRK2), parkin (PARK2), PTEN-induced putative kinase 1 (PINK1), and DJ-1 (PARK7) are linked to familial PD in various populations. Mutations in $L R R K 2$ are most commonly associated with sporadic and familial late-onset PD (117). LRRK2 is a large multidomain protein that regulates several cellular processes, including neurite outgrowth and synaptic morphogenesis, membrane trafficking, autophagy, and protein synthesis, and has been considered a therapeutic target for PD. Mutations in PARK2, $P I N K 1$, and $D J-1$ are associated with autosomal recessive forms of PD and tend to have an earlier age of onset (458). Other genes linked to autosomal dominant forms of PD include SNCA, LRRK2, vacuolar protein sorting 35 (VPS35), DNAJC13 (encodes a chaperone protein named receptormediated endocytosis 8), and coiled-coil-helix-coiled-coilhelix domain containing 2 (CHCHD2), among several others.

1. Redox stress in PD. Dysregulation of redox homeostasis occurs at multiple levels in PD (Fig. 9). A strong link between oxidative stress and cell death of dopaminergic neurons has been established. DA is synthesized from tyrosine by the action of tyrosine hydroxylase, which requires iron as a cofactor, and aromatic amino acid decarboxylase (471). Loss of DA leads to symptoms such as resting tremor, rigidity, bradykinesia, sleep disorder, cognitive deficits, and depression (429). PD has been associated with decreased levels of GSH and other thiols, which are vital for the maintenance of redox balance $(387,391,392$, 523). The generation and sources of ROS in PD include the metabolism of DA, mitochondrial dysfunction, iron deposition, inflammation, aberrant calcium handling, and aging. PD-associated gene products, including DJ-1, PINK1, parkin, alpha-synuclein, and LRRK2, also impact mitochondrial function leading to augmented ROS generation and susceptibility to oxidative stress. In addition, cellular homeostatic processes, including the ubiquitin-proteasome system and mitophagy, are impacted by oxidative stress. On the contrary, increased uptake of DA itself can cause oxidative stress (335). It has been observed that injection of DA into the rat striatum resulted in loss of dopaminergic cells, which could be rescued by antioxidant coinjection (212). Preventing DA degradation caused accumulation of cytosolic DA and caused neurotoxicity, while blocking the conversion of L-DOPA to DA, decreased cytosolic DA, and prevented neurotoxicity (355). High concentrations of DA lead to increased accumulation of oxidation products of DA $(492,493,572)$. Adducts of L-DOPA, DA, and DOPAC with cysteine have been identified in the substantia nigra of PD patients. DA oxidizes more readily than other catecholeamines, and its products such as $\mathrm{N}$-acetyldopamine elicit toxicity by reacting with cysteine residues on proteins in neuronal cells (184). DA is metabolized by monoamine oxidase (MAO) and also autoxidized (572). The autooxidation of DA in vivo generates dopamine ortho-quinone, $\mathrm{O}_{2}{ }^{\bullet-}$ radicals, $\bullet \mathrm{OH}$ radicals, and $\mathrm{H}_{2} \mathrm{O}_{2}$, which are responsible for the cytotoxic effects of DA. The DA quinones or DA semiquinones generated from DA cause damage in cells (493). These quinones can act on proteins such as $\alpha$ synuclein, Parkin, DJ-1, SOD2, and UCH-L1 and affect their activity (48). The oxidized DA products also inhibit the dopamine transporter itself and impair mitochondrial complex I activity $(171,534)$. DA quinones can be oxidized to aminochrome, whose redox reactions result in formation of $\mathrm{O}_{2}{ }^{--}$and reduction of NADPH, forming neuromelanin, which accumulates in the SNpc. Neuromelanin is a dark, insoluble pigment that gives the substantia nigra its 


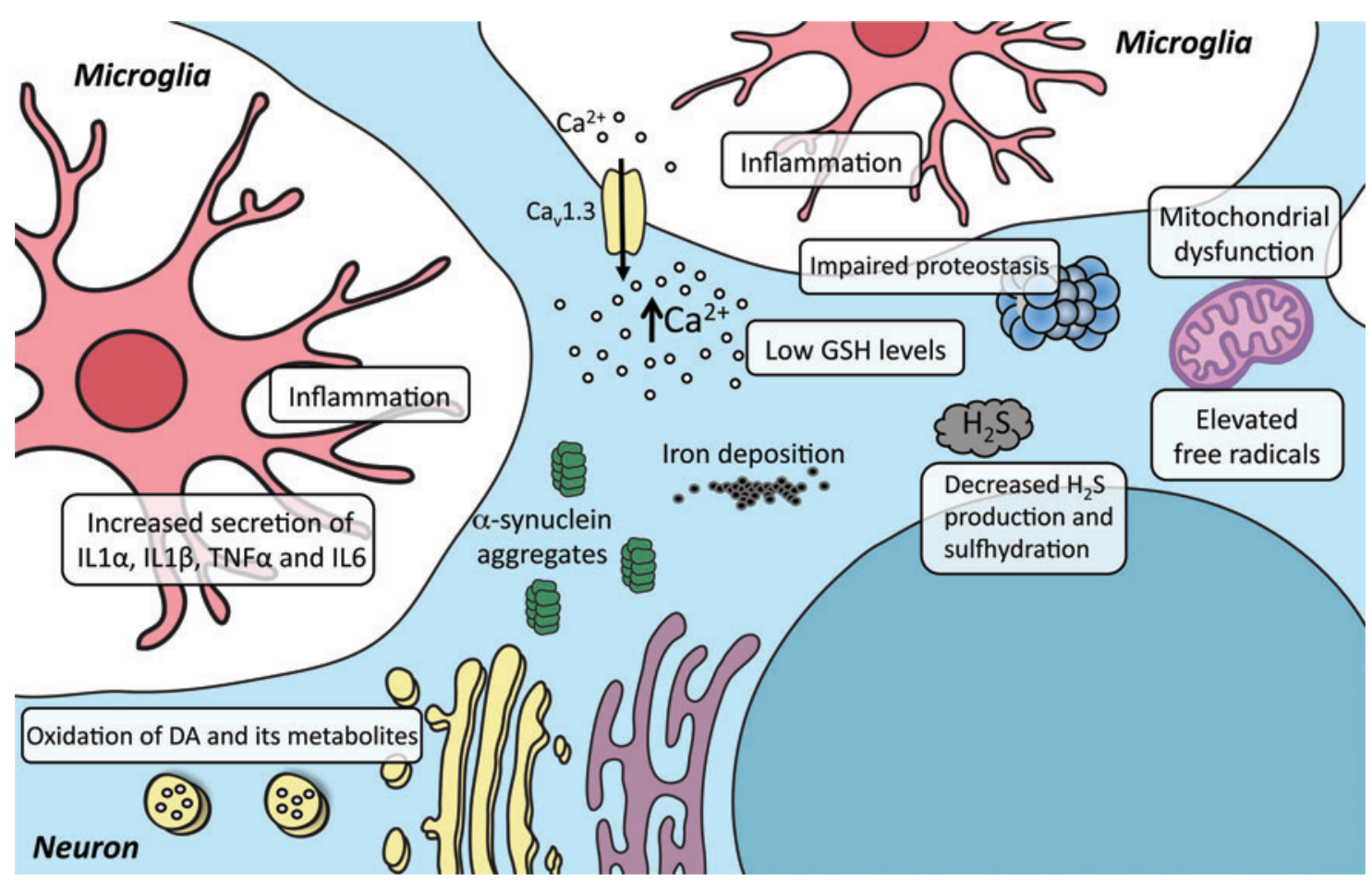

FIG. 9. An overview of events leading to redox imbalance and neurotoxicity in PD. PD affects dopaminergic neurons and is characterized by aggregation of $\alpha$-synuclein, which impairs multiple cellular processes. Impaired mitochondrial function, iron deposition, and low GSH levels in addition to oxidation of DA and its metabolites contribute to elevated levels of free radicals. Increased $\mathrm{Ca}^{2+}$ influx and inflammation with elevated levels of proinflammatory cytokines such as IL1 $\alpha$, IL1 $\beta$, IL6, and TNF $\alpha$, which are secreted by microglia, are observed in PD. In addition to these effects, aggregation of $\alpha$-synuclein affects proteosomal function and impairs clearance of misfolded or damaged proteins, mediating neurotoxicity. DA, dopamine; TNF $\alpha$, tumor necrosis factor $\alpha$. Color images are available online.

name. Intraneuronal neuromelanin may be cytoprotective, defending cells from toxins, metals, and excess catecholamines. In contrast, neuromelanin, released by degenerating neurons, triggers neuroinflammatory processes such as microglial activation. The SNpc is especially vulnerable to toxicity as it has the highest density of microglia in the brain (267).

Modified adducts of DA derived from docosahexaenoic acid (C22:6/omega-3) and arachidonic acid (C18:4/omega$6)$, the major PUFAs in the brain, can mediate neurotoxicity. Of these, hexanoyl dopamine (HED), an arachidonic acidderived adduct, is extremely toxic to human dopaminergic neuroblastoma SH-SY5Y cells. Generation of ROS and mitochondrial dysfunction has been linked to HED-induced cell death (309).

2. Aggregation of $\alpha$-synuclein. A hallmark of PD is the deposition of $\alpha$-synuclein in the cytoplasm of certain neurons in several regions of the brain (51). Aggregated $\alpha$-synuclein is a constituent of Lewy bodies $(181,479)$, which initially accumulate in cholinergic and monoaminergic brainstem neurons and also in the neurons of the olfactory region. As the disease progresses, Lewy bodies are also found in limbic and neocortical brain regions. Mutations in the $\alpha$-synuclein gene, $S N C A$, confer an increased risk for PD (401). The A53T mutant of $\alpha$-synuclein accelerates its aggregation and disease progression (106). Inflammation also contributes to aggregation of $\alpha$-synuclein, including the wild-type protein (528). The function of $\alpha$-synuclein, a 140 amino acid protein, is not well understood, but has been linked to modulation of mitochondrial morphology and function, protein chaperone function, and intracellular trafficking. Similar to most neurodegenerative diseases involving protein aggregation, soluble oligomeric forms of $\alpha$-synuclein are thought to be the toxic species $(522,536)$. Both soluble and fibrillar synuclein bind to metal ions to induce oxidative stress (124). $\alpha$ Synuclein can also bind to proteins and alter their activity or conformation. Aggregated $\alpha$-synuclein binds to SOD1 and increases its aggregation (215). The aggregated $\alpha$-synuclein also binds to tyrosine hydroxylase and inhibits its activity (388). Complex I activity of mitochondria is affected by soluble prefibrillar $\alpha$-synuclein via $\mathrm{Ca}^{2+}$-mediated mitochondrial swelling and depolarization and cytochrome c release. (320). These findings are in agreement with recent findings that administration of fibrillated $\alpha$-synuclein to primary ventral midbrain neuron cultures decreased synaptic protein levels, caused alterations in axonal transport-related proteins, and DNA damage. Mitochondrial impairment (including modulation of mitochondrial dynamics-associated protein content) enhanced oxidative stress, and an inflammatory response was also observed in these studies (505). Activating stress-responsive pathways regulated by the master regulator of antioxidant response genes, Nrf2, rescues neurotoxicity by reducing levels of $\alpha$-synuclein by promoting its degradation (474).

3. Mitochondrial dysfunction in PD. Evidence for the involvement of mitochondrial dysfunction in PD first came from symptoms developed by users of drugs, which were 
identified as 1-methyl-4-phenyl-1,2,5,6-tetrahydropyridine (MPTP) with trace amounts of 1-methyl-4-phenyl-4propionoxy-piperidine that appeared to damage neurons of the substantia nigra (289). Within hours of publication of this discovery, MPTP, which was a promising and valuable tool to study PD, was sold out, as declared by its manufacturer Sigma (288). MPTP is a by-product during the chemical synthesis of the opiate meperidine (571). Later it was shown that MPTP is converted to the toxic metabolite, 1-methyl-4phenylpyridinium or $\mathrm{MPP}^{+}$, which selectively damages the dopaminergic regions of the brain, destroying neurons in the substantia nigra $(290,328)$. Autoradiography using $\left[{ }^{3} \mathrm{H}\right] \mathrm{MPP}^{+}$ in slices of rat brain shows high densities in the caudateputamen and nucleus accumbens (245). It was later shown that MAO converts MPTP into $\mathrm{MPP}^{+}$(92). Later MAO B, but not MAO A, was identified as the specific enzyme involved in this conversion (214). Once formed, $\mathrm{MPP}^{+}$is enriched in mitochondria, where it inhibits the mitochondrial complex I of the ETC (112,413-415). Thus, $\mathrm{MPP}^{+}$causes decline in adenosine triphosphate (ATP) levels and also induces oxidative stress by redistribution of DA $(313,314)$.

Complex I defects have been widely observed in PD. Analysis of postmortem samples from PD patients revealed a decrease in complex I in the substantia nigra and prefrontal cortex $(379,455)$. The components of complex I also exhibited increased oxidative damage as assessed by increased protein carbonylation, a marker of oxidative damage (261). Reduction in complex I activity in PD has also been reported in several other studies $(170,261)$. Exposure to the pesticide, rotenone, which inhibits complex I has been linked to an increased risk of developing PD (258). Dopaminergic neurons are highly vulnerable to complex I inhibitors whose toxicity is partly mediated by generation of ROS (192, 334, 430). Studies have revealed that inhibition of complex I is not sufficient to induce cell death of dopaminergic neurons (96). In addition to generation of oxidative stress, failure of mitochondrial bioenergetics to generate ATP has been observed in PD. Several studies have reported that cell death caused by inhibition of complex I in PD cannot be fully ascribed to ROS or prevented by antioxidants as complex I inhibitors have other effects such as destabilization of the cytoskeletal network in addition to other effects such as activation of inflammatory pathways $(27,70,142)$.

In addition to suboptimal electron transport in PD, abnormal mitochondrial homeostasis has also been observed in PD (397). Healthy neurons remove damaged mitochondria by a quality control process termed mitophagy involving the PDlinked proteins PINK1 and parkin. The loss of mitochondrial membrane potential causes recruitment of PINK1 accumulation to the outer mitochondrial membrane. PINK1 recruits the E3 ubiquitin ligase, parkin, to the mitochondria leading to the ubiquitination of mitochondrial membranes, and removal of the defective mitochondria (364). Loss of parkin or PINK1 compromises the ability to remove damaged mitochondria leading to an accumulation of these dysfunctional organelles leading to early-onset PD.

4. Iron accumulation in PD. Iron accumulation in PD was observed as early as 1924 (301). Initial studies utilized Prussian blue staining to localize iron deposits (138). Nuclear magnetic resonance studies have revealed that iron deposition and striatal atrophy are associated with disease pro- gression in PD (225). Iron deposition is not unique to PD, as elevated iron content is also observed in AD, HD, and ALS prompting an additional categorization of diseases involving iron: the neurodegeneration with brain iron accumulation syndromes. In PD, iron deposits are especially high in the substantia nigra. The accumulation of iron is directly correlated with disease severity and motor deficits (333). Elevated iron levels can mediate aggregation of $\alpha$-synuclein (515). Proteins involved in iron metabolism are dysregulated in PD. Expression of ferritin, the iron binding protein, is diminished in PD patients. On the contrary, the transporter of iron, the divalent metal ion transporter, is upregulated (444). In addition, ceruloplasmin, an iron export ferroxidase, is decreased by more than $80 \%$ in the substantia nigra of idiopathic PD brains. Ceruloplasmin knockout mice have elevated iron levels and develop some symptoms of PD, which can be rescued by iron chelation. Similarly, in the MPTP chemical model of PD, infusion of ceruloplasmin decreases neurodegeneration (20). One of the upstream events and contributing factors to iron accumulation is nitrosative stress (403). The inhibitor of NO synthase, 7nitroindazole, prevents MPTP-induced toxicity in mice (461). The expression of $\beta$-APP, which promotes neuronal iron export, is decreased in dopaminergic neurons of the substantia nigra of PD brains. In agreement with these findings, $\mathrm{APP}^{-/-}$mice develop iron-dependent nigral cell loss (20). Recently, a new form of cell death involving iron, ferroptosis, was identified (131). Initiation of ferroptosis is specific to intracellular iron, but not other metals, and has been shown to be genetically, morphologically, and biochemically distinct from apoptosis, necrosis, and autophagy $(131,550)$. Although ferroptosis has several features similar to oxytosis, another form of cell death mediated by glutamate toxicity, there are several differences (550). In oxytosis, elevated calcium ions activate a number of serine proteases, calpains, and phospholipases, leading to mitochondrial damage and nuclear translocation of AIF (apoptosis-inducing factor). These changes are necessary for executing oxytosis, whereas they are dispensable for ferroptosis. Thus, it has been suggested that oxytosis may be a combination of the more general ferroptosis and a specialized type of damage induced by glutamate. In ferroptosis, depletion of cysteine or GSH can initiate the process due to elevation of iron. Lipid peroxidation is a consequence of ferroptosis induction. Induction of ferroptosis due to cysteine depletion causes degradation of ferritin (i.e., ferritinophagy), the iron binding protein, which releases iron via the nuclear receptor coactivator 4-mediated autophagy pathway (292). Ferroptosis was characterized in Lund human mesencephalic cells and confirmed in organotypic slice cultures ex vivo and in the MPTP mouse model. The cell death pathway in this instance differs from the classical ferroptosis reported previously, in that activation of protein kinase $\mathrm{C} \alpha(\mathrm{PKC} \alpha)$ can initiate ferroptosis. Ferroptosis inhibitors, such as ferrostatin-1, are beneficial in PD models (132). Targeting iron disposition in PD therefore is being explored to manage disease symptoms $(112,129)$.

5. Dysregulated calcium signaling in PD. Several studies show that disturbed $\mathrm{Ca}^{2+}$ contribute to neurotoxicity in PD $(496,560)$. Because of its involvement in cellular processes ranging from the regulation of enzyme activity to programmed cell death, calcium is under very tight homeostatic 
control. L-type $\left(\mathrm{Ca}_{\mathrm{v}} 1\right.$ family) voltage-gated $\mathrm{Ca}^{2+}$ channels, $\mathrm{Ca}_{\mathrm{v}} 1.2$ and $\mathrm{Ca}_{\mathrm{v}} 1.3$, have been implicated in PD. In adult dopaminergic neurons, $\mathrm{Ca}_{\mathrm{v}} 1.3$ is preferentially used as the neurons age, for $\mathrm{Ca}^{2+}$ influx and support of rhythmic pacemaking activity, important for maintaining basal DA levels in the striatum although these neurons have a decreased ability to handle elevated $\mathrm{Ca}^{2+}$ influx $(233,495)$. Activity-dependent $\mathrm{Ca}^{2+}$ influx has been shown to elevate mitochondrial oxidative stress in DA neurons, which can explain their selective vulnerability $(134,198)$. Increased expression of $\mathrm{Ca}_{\mathrm{v}} 1.3$ in early-stage PD, before the appearance of pathological changes, also suggests the disruption of $\mathrm{Ca}^{2+}$ homeostasis (233). Consistent with these findings, isradipine, an L-type $\mathrm{Ca}^{2+}$ channel blocker, which is now in clinical trials, prevents cell death of mouse DA neurons, challenged with $\alpha$-synuclein, MPTP, or 6-hydroxydopamine (6-OHDA) (82, 237, 527). In addition to these mechanisms, $\mathrm{Ca}^{2+}$ dysregulation contributed by intracellular stores, such as the ER and mitochondria, has been implicated $(71,560)$.

6. Inflammation in PD. PD is also characterized by microglial activation, accumulation of proinflammatory cytokines, and chronic inflammation $(179,351)$. Analysis of postmortem brains of PD patients revealed the presence of macrophages and microglia in the SN (220, 342). Early studies revealed that administration of lipopolysaccharides (LPSs) into the brain of mice and rats selectively induces cell death of dopaminergic neurons $(77,216)$. Mice overexpressing $\alpha$-synuclein developed persistent neuroinflammation after LPS challenge, with prolonged microglial activation, expressing iNOS and NOX enzymes that generate ${ }^{\bullet} \mathrm{NO}$ and $\mathrm{O}_{2}{ }^{\bullet-}$, respectively (168). Thus, activation of microglia contributes to elevation of free radicals as demonstrated by these and other studies (564). In these studies, inhibition of iNOS and NOX enzymes blocked pathology and neurodegeneration. The contribution of $\alpha$-synuclein to inflammatory processes has also been demonstrated in microglial cell lines, where exposure to extracellular $\alpha$-synuclein led to in- creased secretion of the cytokines IL $1 \alpha$, IL $1 \beta$, TNF $\alpha$, and IL6 (12). A possible mechanism underlying activation of microglia by $\alpha$-synuclein involves Nurr1, an orphan nuclear receptor belonging to (NR)4 family of orphan nuclear receptors. Nurr1 has been reported to inhibit expression of proinflammatory neurotoxic mediators in both microglia and astrocytes (442). Levels of Nurr1 have been shown to be decreased in human PD, and mutations resulting in reduced expression of Nurr1 are associated with late-onset familial PD (179, 352).

7. Dysregulated gasotransmitter signaling in PD. Disrupted gasotransmitter signaling involving $\mathrm{H}_{2} \mathrm{~S}$ and ${ }^{\bullet} \mathrm{NO}$ has been associated with PD. The decrease of endogenous $\mathrm{H}_{2} \mathrm{~S}$ production in the substantia nigra of 6-OHDA-treated rats reveals a link between $\mathrm{H}_{2} \mathrm{~S}$ and $\mathrm{PD}$ (227). Accordingly, administration of the $\mathrm{H}_{2} \mathrm{~S}$ donor, $\mathrm{NaHS}$, reverses motor deficits, reduces loss of tyrosine hydroxylase-positive neurons in the substantia nigra and diminishes markers of oxidative stress such as the elevated MDA levels in 6-OHDA-treated rats. $\mathrm{H}_{2} \mathrm{~S}$ also attenuates 6-OHDA-induced NOX activation. In $\mathrm{PD}$, altered nitrosylation and sulfhydration of parkin, an E3ubiquitin ligase, mediated by ${ }^{\bullet} \mathrm{NO}$ and $\mathrm{H}_{2} \mathrm{~S}$, have been observed. The activity of parkin is inhibited, by nitrosylation, leading to aggregation of its substrate $\alpha$-synuclein and neurotoxicity (99) (Fig. 10). In contrast, sulfhydration activates parkin to degrade misfolded proteins and promote neuroprotection (519). In postmortem PD brains, sulfhydration of parkin is diminished and nitrosylation increased (519). Overexpressing cystathionine $\beta$-synthase (CBS), one of the biosynthetic enzymes of $\mathrm{H}_{2} \mathrm{~S}$, increases parkin sulfhydration and E3 ubiquitin ligase activity. Administration of $\mathrm{H}_{2} \mathrm{~S}$ donors has proved beneficial in PD. Inhalation of $\mathrm{H}_{2} \mathrm{~S}$ by MPTP mice ameliorates symptoms and reduces the death of dopaminergic neurons (266). Treating the 6-OHDA-induced PD mouse model with an $\mathrm{H}_{2} \mathrm{~S}$ releasing L-DOPA derivative ACS84 stimulates the antioxidant defense pathway regulated by Nrf2, preserves dopaminergic neurons, and confers

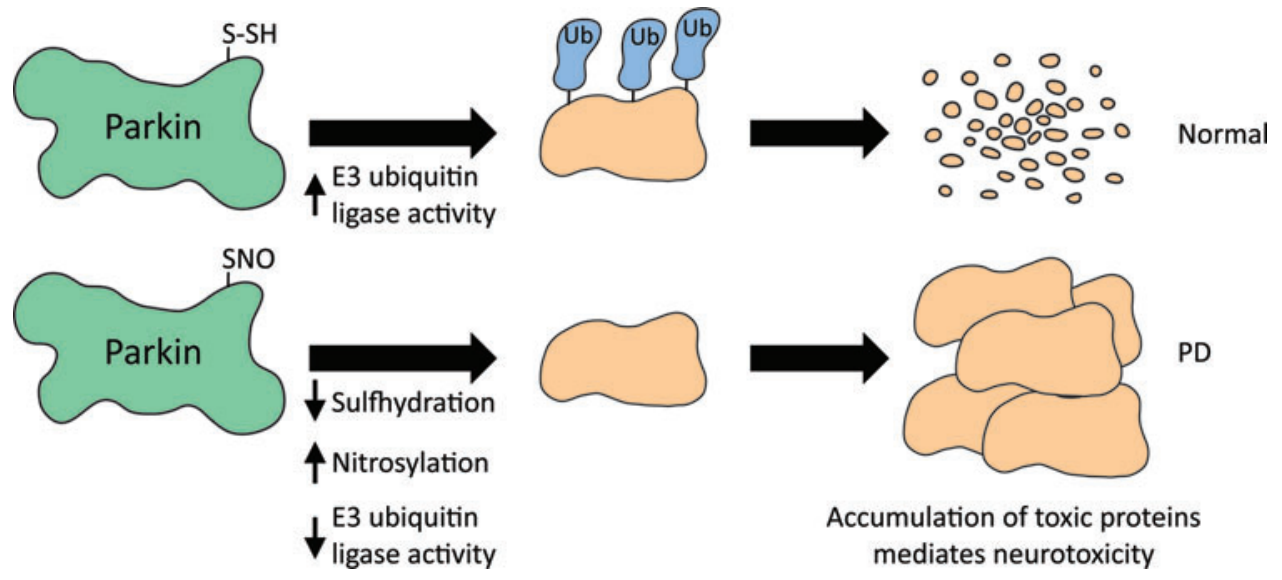

FIG. 10. Sulfhydration and nitrosylation exert opposite effects on parkin. In PD, aggregation of $\alpha$-synuclein to form Lewy bodies is a pathogenic hallmark. Enzymes such as parkin, which is an E3 ubiquitin ligase, clear misfolded and aggregated proteins under normal conditions. The activity of parkin is increased by sulfhydration/persulfidation of reactive cysteine residues, whereas nitrosylation has the opposite effect. In PD, decreased sulfhydration and increased nitrosylation of parkin were observed, leading to impaired ubiquitination and proteosomal degradation of $\alpha$-synuclein and other substrates. Color images are available online. 
neuroprotection (547). Protective effects of $\mathrm{H}_{2} \mathrm{~S}$ have also been reported in cell culture models of PD (510).

Nitrosylation of parkin also affects other pathways. As parkin is a suppressor of Drp1 expression, which is involved in mitochondrial fission, its nitrosylation abrogates this function. As a result, upregulation of Drp1 occurs with associated abnormal mitochondrial dynamics (568). Similarly, S-nitrosylation of Prx2, a peroxidase that plays important roles in regulation of oxidative stress, inhibits its enzymatic activity and antioxidant defense. Increases of nitrosylated Prx2 have been detected in mouse models of PD as well as human PD (494). Ubiquitin C-terminal hydrolase-1/UCHL1, a deubiquitinating enzyme and a constitutent of Lewy bodies, has been shown to be nitrosylated in vitro. Specific nitrosylation of Cys90, Cys152, and Cys220 occurs, altering the enzymatic activity and structural stability. Nitrosylation destabilizes its structure leading to the formation of an amorphous aggregate, which acts as a nucleating site for native $\alpha$-synuclein and accelerated aggregation (283). Thus, protein S-nitrosylation can be targeted in neurodegenerative diseases to improve survival and outcome (363). For instance, thioredoxin-mimetic peptides, which catalyze the reduction of SNO, protect cells from nitrosative stress (280). Another example is the use of nitromemantine, which not only blocks overactivated NMDARs but also acts as an NO donor to nitrosylate the receptor and further inhibit its activity $(500,501)$.

\section{Amyotrophic lateral sclerosis}

1. ALS and redox imbalance. ALS is a neurodegenerative disease manifesting selective degeneration of upper and lower motor neurons (507). In the United States, ALS is referred to as Lou Gehrig's disease after the famous baseball player who succumbed to the disease. About $90 \%$ of ALS cases are sporadic, whereas the remaining (10\%) are familial (439). The familial causes include mutations in the genes $\mathrm{Cu}$ Zn superoxide dismutase 1 (SOD1), Tar DNA binding protein (TARDP), fused in sarcoma (FUS), ubiquilin 2 (UBQLN2), $V C P$, and optineurin $(O P T N)$ among others. Other proteins mutated in ALS include senataxin, ataxin2, HNRNPA2/B1, ELP3, HNRNPA1, alsin, FIG4, VABP, and CHMP2B (327). Similar to most other neurodegenerative disorders, several factors, including oxidative stress, have been observed in ALS (Fig. 11). GSH content in the motor cortex of ALS patients is decreased, which can promote oxidative stress (531). In addition, mitochondrial function is affected in ALS. Mitochondrial dysfunction in ALS was first identified as morphological abnormalities in the skeletal muscle, liver, spinal cord neurons, and motor cortex during postmortem analysis of ALS patients and cell culture models (219). The morphological changes are accompanied by decreased activities of complexes II and IV of the mitochondrial ETC and oxidative damage to mitochondrial protein and lipid components $(338,345)$.

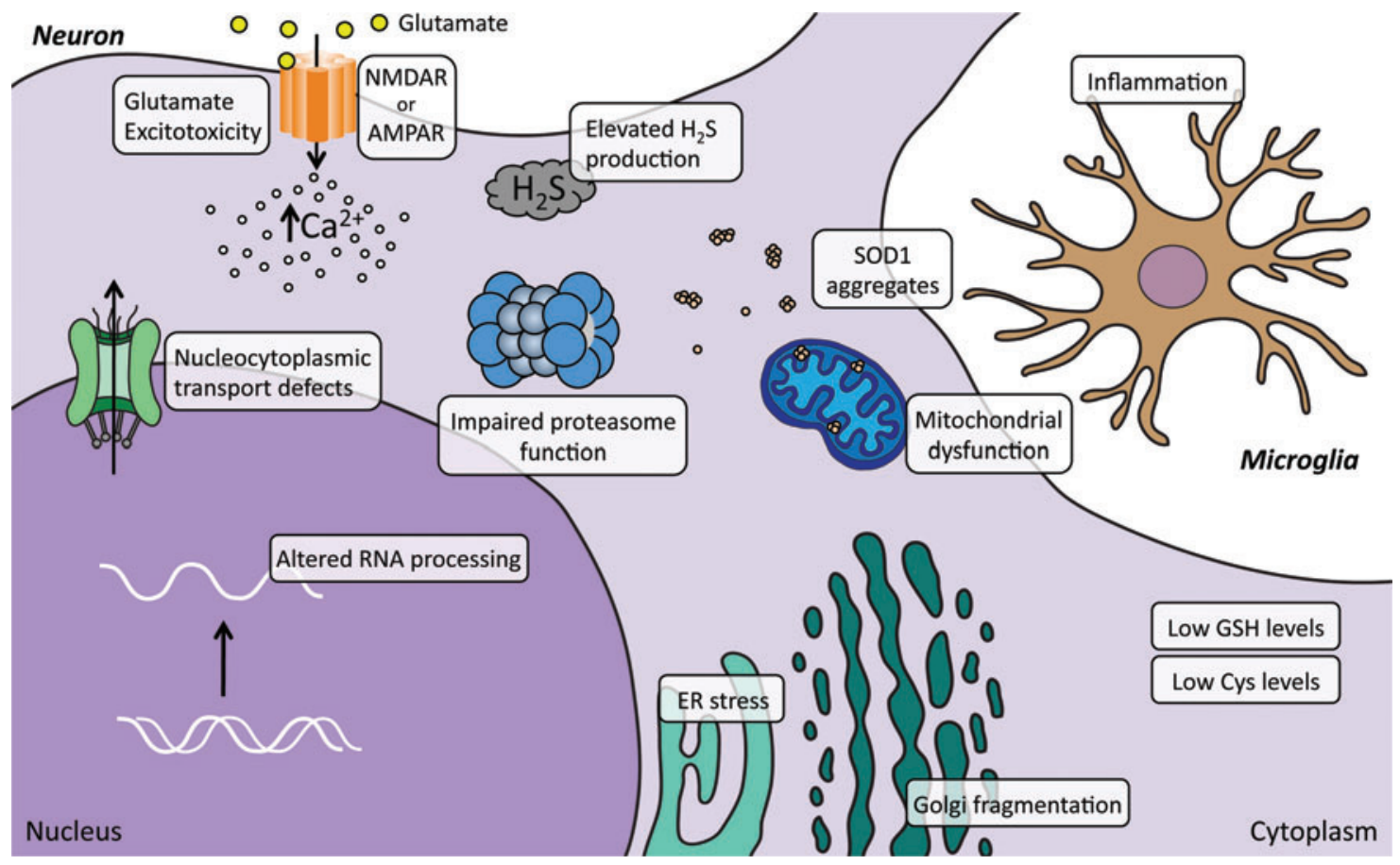

FIG. 11. Pathways disrupted in ALS. ALS can be triggered by several mutations, which can elicit cytotoxicity. In ALS caused by mutations in SOD1, misfolded aggregates of SOD1 impair proteostasis by inhibiting proteasomal function. Mitochondrial function is also impaired, which can lead to elevated oxidative stress. In addition, ER stress, Golgi fragmentation, inflammation, and increased microglial accumulation are also observed. The G93A SOD1 mutation has also been shown to increase $\mathrm{H}_{2} \mathrm{~S}$ production, which is toxic at high levels. ALS is also associated with impaired glutamate transport leading to overstimulation of the NMDA or $\alpha$-amino-3-hydroxy-5-isoxazole propionate receptors (AMPA) causing $\mathrm{Ca}^{2+}$ influx and cell death. Aberrant RNA processing in addition to defects in nucleocytoplasmic trafficking is also affected in several cases of ALS, such as those caused by hexanucleotide repeat expansion in the C9ORF72 open reading frame. C9ORF72, chromosome 9 open reading frame 72; ER, endoplasmic reticulum; NMDA, N-methyl D-aspartate; SOD1, superoxide dismutase 1. Color images are available online. 
2. ALS and mutations in SOD1. Among the familial cases of ALS, $\sim 20 \%$ are caused by dominantly inherited mutations in the SOD1 gene. More than a hundred mutations have been identified in the SOD1 gene, but not all of the mutants have been characterized. The most commonly studied genetic mutation in SOD1 is the G93A mutation, which is a gain-of-function mutant that causes degeneration of motor neurons $(197,433)$. The mutation causes the protein to aggregate and mediate toxicity. Transgenic mice expressing human SOD1 harboring the G93A mutation become paralyzed in one or more limbs, which arises due to motor neuron loss from the spinal cord, and die within 5-6 months of age (197). Human neuroblastoma SH-SY5Y cells expressing G93A SOD1 display an increase in both cytosolic and mitochondrial ROS production, which can be mitigated by NAC, which also serves as a precursor for GSH biosynthesis (42). Oxidative stress has been associated with disease progression in ALS caused by SOD1 mutations, but the precise mechanisms by which motor neurons degenerate have not been well characterized. Cysteine residues have been implicated in the aggregation of SOD1 (111). Human SOD1 has four cysteine residues, of which two, Cys57 and Cys146, form an intramolecular disulfide bond (499). Cys57 and Cys 146 are highly conserved in yeast, plants, flies, fishes, and mammals. The other two cysteine residues Cys6 and Cys111, which are not conserved, do not form a bridge. SOD1 proteins aggregate when they are in the metal-free form and, depending on the mutation, rates of oligomerization vary. These oligomers are formed through oxidation of Cys6 and Cys111 and are stabilized by hydrogen bonds, between beta strands, forming amyloid-like structures. Cys111 is present in human and ape but not mouse SOD1. Cys111 is surface exposed, reactive, and prone to modifications. Cys 111 has a relatively low $\mathrm{p} K_{\mathrm{a}}$, leading to its ionization at physiological $\mathrm{pH}$, which facilitates its modification by a wide variety of reactive cysteine interacting compounds $(56,68)$. Cys111 was shown by mass spectrometry to be modified by a persulfide group (121). Modified SOD1 is more resistant to oxidation-induced aggregation caused by copper and $\mathrm{H}_{2} \mathrm{O}_{2}$. Crystallographic analysis confirms that Cys 111 is modified by a covalent polyheptane sulfane sulfur (559). Sulfane sulfur is elemental sulfur that has six valence electrons with no charge, which can bond together to form hydropersulfides (R-S-SH) and polysulfides (-S-S $\left.{ }_{n}-\mathrm{S}-\right)$. Sulfane sulfur can be derived from $\mathrm{H}_{2} \mathrm{~S}$ and act as a signaling molecule in vivo $(5,268,470)$. The interaction of SOD1 with $\mathrm{H}_{2} \mathrm{~S}$ has been reported to increase its activity (462). Cys111 also plays a role in the mitochondrial localization of SOD1 (147). SOD1 enters the mitochondria as the metal-free form. In order for SOD1 to be targeted to mitochondria, interaction with the protein, CCS, which is also redox sensitive, is required (259). Oligomers formed by mutant SOD1 proteins associated with the mitochondria cause a shift in the redox state of the organelle and impairment of respiratory complexes in the motor neuron cell line NSC34. Association of mutSOD1 with mitochondria decreases the reduced/oxidized glutathione ratio (GSH/GSSG). However, NSC-34 cells expressing Cys6F/Cys111S mutSOD1 do not exhibit alteration of the GSH/GSSG ratio in the mitochondria and impairment of the respiratory chain, indicating that a link exists between mitochondrial localization and toxicity of mutSOD1. In another study, generation of the H46R SOD1-expressing mouse model of ALS where Cys111 is mutated delayed disease progression (361).

In addition to these changes, elevated $\mathrm{H}_{2} \mathrm{~S}$ production has been reported in ALS (120). Increased levels of $\mathrm{H}_{2} \mathrm{~S}$ are detected in the tissues of the G93A mouse model of ALS and in media of spinal cord cultures of these mice. Elevated $\mathrm{H}_{2} \mathrm{~S}$ was also detected in cerebrospinal fluid of ALS patients, indicative of aberrant gasotransmitter signaling in ALS. Decreased cysteine levels have been reported in the plasma of G93A mice (25). Supplementation of the diet with a cysteinerich whey isolate rescues GSH content in tissues and delays disease onset in the G93A mice (434). The involvement of redox imbalance in ALS is further supported by studies involving the G93A mice on a gclc deleted background. GCLC (glutamate cysteine ligase light chain) participates in the biosynthesis of GSH; its deletion leads to decreased antioxidant capacity, mitochondrial dysfunction, and accelerated disease progression of ALS (520).

3. The C9ORF72 model of ALS. One of the leading causes of ALS as well as frontotemporal dementia (FTD) is a mutation in the noncoding region of the chromosome 9 open reading frame 72 ( $C 9 O R F 72$ ) locus, where an expansion of the hexanucleotide GGGGCC occurs $(125,201,324,421)$. The repeat expansion was found in $11.7 \%$ of familial FTD and $23.5 \%$ of familial ALS (125). The wild-type C9ORF72 allele typically has less than 20-25 copies of these hexanucleotide repeats, whereas the C9ORF72 ALS/FTD patients can have repeats up to thousands $(125,176,421)$. One of the first models of ALS with an expanded hexanucleotide repeat (generated by transgene delivery mediated by somatic transduction of adeno-associated virus [AAV] carrying the G4C2-repeat DNA to the central nervous system) resulted in mice that had deposition of the dipeptide repeats in the brain. In addition, these mice displayed cortical neuron and cerebellar Purkinje cell loss, astrogliosis, and lower body weight in addition to behavioral abnormalities, including hyperactivity, anxiety, antisocial behavior, as well as motor deficits (90). Bacterial artificial chromosome DNA clones harboring either partial- or full-length C9ORF72 gene with the hexanucleotide repeats were also used to generate transgenic mice, of which only the full-length transgene elicited abnormalities such as nucleolar stress and downregulation of immunomodulatory and extracellular matrix pathways (370, 394). The C9ORF72 protein has roles in membrane trafficking and autophagy $(299,464,530)$. Three mechanisms have been proposed to mediate neurotoxicity: diminished C9ORF72 protein levels, generation of toxic RNA species, and noncanonical translation leading to accumulation of dipeptide repeat proteins (DPRs). Possible reasons for decreased expression of C9ORF72 protein include epigenetic silencing and transcriptional instability $(39,200)$. The expansion causes aberrant translation in all three frames, which leads to production of DPRs such as poly GA, poly GR, and poly GP (Fig. 12). The dipeptide proteins are produced by repeat-associated non-ATG-dependent translation or RAN translation (101). Expression of poly GR in human neurons causes mitochondrial dysfunction by binding to mitochondrial ribosomal proteins, which leads to oxidative stress. Increased DNA damage and oxidative stress have been observed in induced pluripotent stem cell (iPSC)-derived C9ORF72 motor neurons in an age-dependent manner (312). 


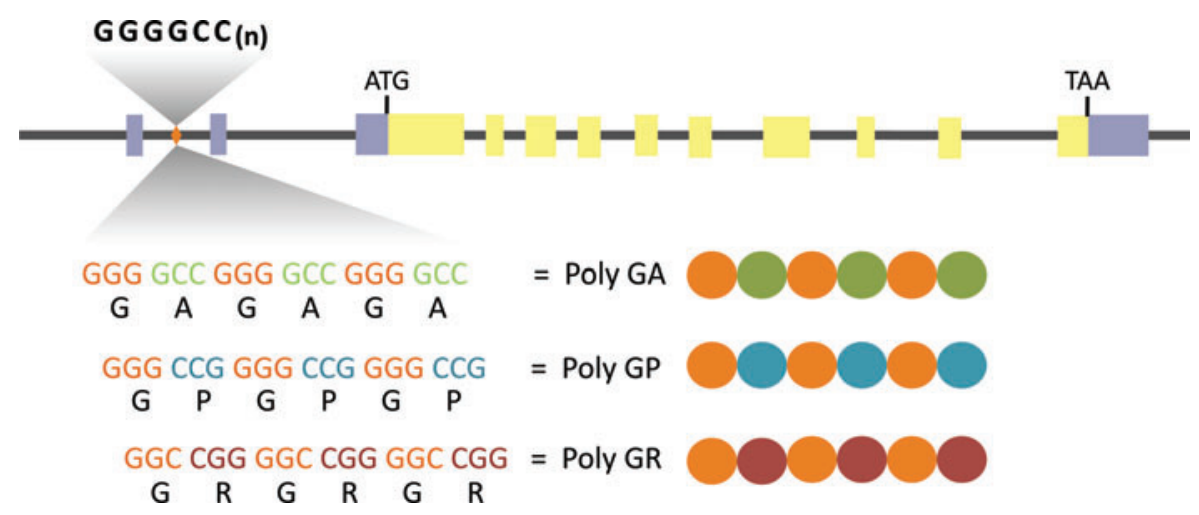

FIG. 12. Production of dipeptide repeat proteins in ALS caused by the C9ORF72 mutation. Mutations in $C 9 O R F 72$ comprise the largest fraction of familial ALS, where an expansion of the hexanucleatide repeat occurs in C9ORF72. Non-ATG initiated translation occurs in all three reading frames, resulting in the formation of the polydipeptide repeats, poly GA, poly GP, and poly GR, which aggregate and affect multiple cellular processes. Of these, the poly GR dipeptides cause oxidative stress. Color images are available online.

The DNA damage increases as a function of age. Transfection of a poly GR construct into wild-type cells induces oxidative stress and DNA damage, and increased toxicity. Mitochondria-generated ROS are also increased in these cells. More importantly, pharmacological or genetic reduction of oxidative stress partially suppresses these detrimental effects in human motor neurons and flies (312).

\section{E. Autism spectrum disorders}

Autism spectrum disorders (ASD) are a group of neurodevelopmental disorders characterized by impaired social interactions, communication, repetitive stereotypic behaviors, and abnormalities in language and perception. Other disorders such as Asperger's syndrome, childhood disintegrative disorder, and pervasive developmental disorders, not otherwise specified, which share certain behavioral features with autism, have been collectively designated as ASD. The exact etiology of the disorders has been elusive, but probably involves genetic components as well as environmental contributions (393). Genetic factors have been challenging to elucidate as they are highly heterogeneous, exhibiting varying gene/gene and gene/ environment interactions with different degrees of penetrance. Thus far, no reliable markers of ASD have been identified. The prevalence of autism is about 7.6 in 1000 (33). ASD patients frequently report comorbidities such as sleep disorders, digestive tract problems, and impairment in motor functions. Regardless of the cause of ASD, oxidative stress and suboptimal antioxidant defense have been linked to autism in several studies $(83,188,242,285)$.

1. Mitochondrial dysfunction and oxidative stress in ASD. The first indication that mitochondrial function may be affected in ASD came from the observation that the levels of lactate are higher in the plasma of autistic subjects, suggesting a deficit in OXPHOS (103). Later it was demonstrated that in addition to high lactate levels, there is an accumulation of Krebs cycle metabolites, impaired glucose utilization, and decreased ATP production (311). Reduced expression of all five electron transport complexes has been observed in autistic individuals, with pronounced defects in complex I (84). In addition, marked increases in lipid peroxidation occur in the cerebellum and frontal cortex of autistic children, which may be linked to abnormal energy homeostasis and mitochondrial dysfunction. Mitochondrial copy number is usually proportional to the cellular DNA content (468). A cell typically harbors 2-10 mitochondria under normal conditions (427). This number can change based on the energy requirements of the cell in response to physiologic stimuli, but may not necessarily correlate with increased OXPHOS. Mitochondrial copy number variation occurs in response to stress and has been observed in leukocytes exposed to oxidative stress caused by alteration of plasma antioxidants/prooxidants and oxidative damage to DNA (307). Mitochondrial abnormalities have also been reported in cell lines derived from autistic patients (432). Higher expression of the mitochondrial fission proteins, Fis 1 and Drp1, and lower levels of the fusion proteins, Mfn1, Mfn2, and Opa1, have been observed in ASD patients (503). Other studies report decreased levels of mitochondrial SOD2 and elevated oxidative DNA damage. Literature review and metaanalysis revealed decreased blood levels of GSH, glutathione peroxidase, methionine, cysteine, and an increase in oxidized glutathione, GSSG, in autistic patients relative to controls (163). Lymphoblastoid cell lines from children with ASD display higher oxidative stress and decreased GSH levels (431). Increases in cystathionine levels and decreased cysteine and methionine levels have also been observed in the plasma of autistic patients, which could contribute to oxidative stress, as the cysteine/cystine redox couple plays a central role in maintenance of redox balance (23). In addition to low GSH levels, low levels of vitamin B12 have been reported in autism and other neuropsychiatric disorders (566). It remains to be clarified whether altered redox control in patients contributes to ASD. More recently, deficits in the molybdenum cofactor sulfurase (MOCOS), an enzyme involved in purine metabolism, have been observed in autism (146). MOCOS sulfurates the molybdenum cofactor, which activates $\mathrm{XDH}$ and aldehyde oxidase 1 (AOX1) (235). Both XDH and AOX1 contribute to redox homeostasis in cells and hence depletion of MOCOS results in elevated oxidative stress.

2. Abnormal transmethylation, reverse transsulfuration, and vitamin B metabolism in ASD. In addition to elevated oxidative stress, abnormal transmethylation has been linked to 
the pathophysiology of autism. In a study published in 2004, fasting levels of plasma transmethylation and reverse transsulfuration metabolites were found to be abnormal in autistic children (242). The transmethylation pathway and the reverse transsulfuration pathway are linked by the metabolite homocysteine, which is a metabolic decision point, where either cysteine and GSH biosynthesis or S-adenosylmethionine (SAM) and related metabolites can be generated (Fig. 13). Autistic subjects had significantly diminished levels of methionine. Interestingly, a study showed that parents of autistic children have deficits in the methylation and reverse transsulfuration pathway and associated redox imbalance (244). The ratio of $\mathrm{S}$-adenosylmethionine to $\mathrm{S}$-adenosylhomocysteine (SAM/SAH) is decreased indicating altered methylation capacity. These changes can lead to altered epigenetic patterns. Several unbiased genome-wide analyses have implicated genes involved in chromatin remodeling events such as histone demethylation and the recognition of DNA methylation (291). Methylation of the oxytocin receptor (OXTR) promoter is increased in several cases of ASD and is frequently associated with preterm birth, a risk factor for autism (36). OXTR is involved in the regulation response to stress and anxiety, social memory and recognition, and maternal behavior and could underlie some of the behavioral abnormalities observed in autistic patients.

The reverse transsulfuration pathway is also affected in ASD. Total glutathione levels are decreased, with elevations in oxidized glutathione, GSSG, leading to an approximately threefold reduction in the ratio of GSH/GSSG. Cysteine, one of the components of GSH, is diminished, a contributing factor for the low GSH levels and oxidative stress associated with ASD, as the availability of cysteine is the rate-limiting step for GSH biosynthesis. In several studies, elevated homocysteine levels have been observed in autistic children along with reduced $\mathrm{Mg}^{2+}$ levels in hair samples $(242,256$, 380). $\mathrm{Mg}^{2+}$ is an essential cofactor for several enzymes, for utilization of vitamin B6, and for certain ATP-dependent reactions. $\mathrm{Mg}^{2+}$ is also involved in the disposition and metabolism of neurotransmitters, which can affect mood and behaviors. Thus, a decrease in $\mathrm{Mg}^{2+}$ can explain decreased ATP synthesis and the suboptimal transmethylation and transsulfuration activities, which can lead to decreased transcription and synaptic plasticity, a phenomenon termed "Magnesium deficiency hypothesis" (256). Thus, increased homocysteine and decreased $\mathrm{Mg}^{2+}$ levels have been proposed to be diagnostic markers in combination with other symptoms. In addition to these aberrations, deficits in vitamin B12 (cobalamin), a cofactor for enzymes such as methionine synthase and methylmalonylmutase, have been observed in the postmortem frontal cortex of autistic patients, a feature also observed in neuropsychiatric conditions such as schizophrenia and aging (566).

3. Treatments for ASD. Based on the observations linking mitochondrial dysfunction and oxidative stress to ASD, treatments include reducing oxidative stress and improving mitochondrial function (164). These strategies include increasing complex I activity by fatty acid and folate supplementation (126). Trials involving antioxidants have shown promise and include treatments with ascorbate (133) and NAC $(172,210)$. Treatment with methylcobalamin and folinic acid increases cysteine and GSH content in children with autism (243). Other supplements evaluated include Lcarnosine and ubiquinol $(91,199)$.

\section{F. The ataxias}

The word "ataxia" is derived from the Greek words "a" without and "taxis" meaning order. Clinically, ataxia refers to lack of muscle coordination when voluntary movement is attempted. Ataxia results from the damage or degeneration of neurons that control movement typically in the cerebellum (Fig. 4). Most ataxias have a genetic cause and thus far more than 50 ataxias have been identified. As the topic is vast and heterogeneous with respect to underlying causes and mechanisms, only a few ataxias are described (Fig. 14). Ataxias can be those that are hereditary and those that are not. The classification of the various ataxias has been widely debated,

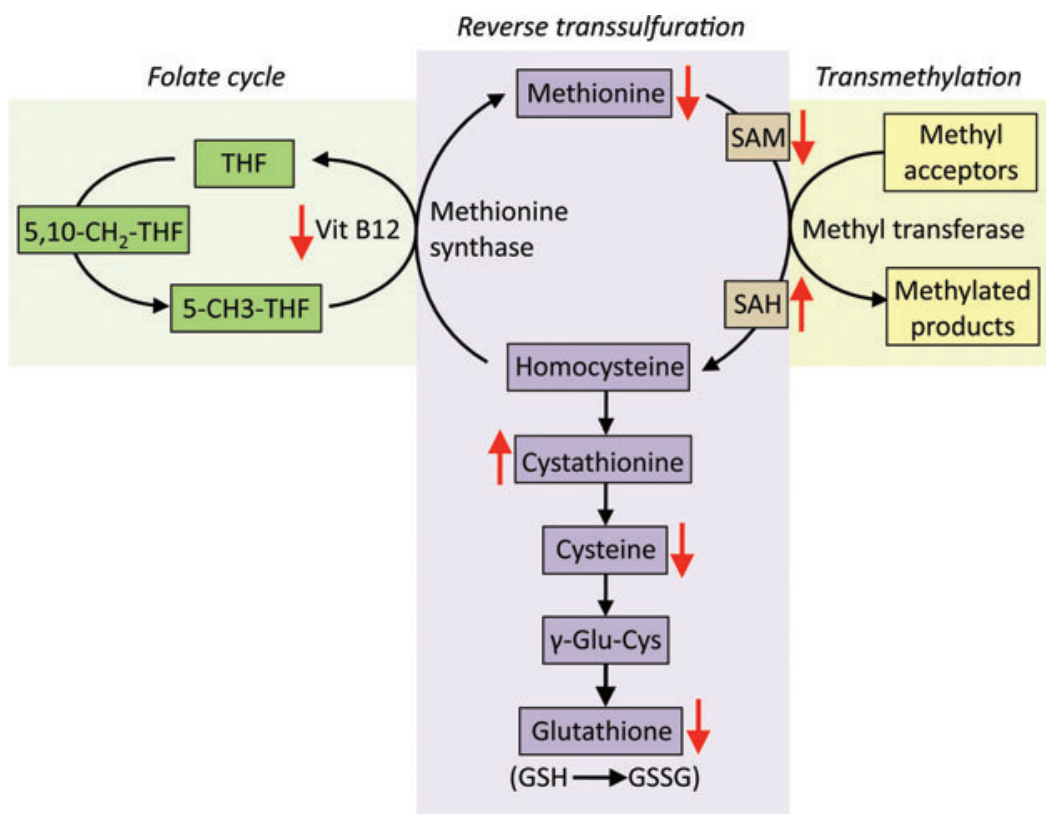

FIG. 13. Aberrant transsulfuration and transmethylation pathway in ASD. The reverse transsulfuration pathway and the transmethylation pathway intersect at the metabolite homocysteine. Compared to normal individuals, the levels of several metabolites in these pathways are altered in ASD (indicated by red arrows). The levels of the major antioxidants, glutathione (GSH) and cysteine, are decreased in ASD, in addition to vitamin B12 and SAM. The ratio of S-adenosylmethionine to $\mathrm{S}$-adenosylhomocysteine ( $\mathrm{SAM} / \mathrm{SAH}$ ) is decreased, which could alter epigenetic patterns. ASD, autism spectrum disorders; SAM, $\mathrm{S}$-adenosylmethionine. Color images are available online. 


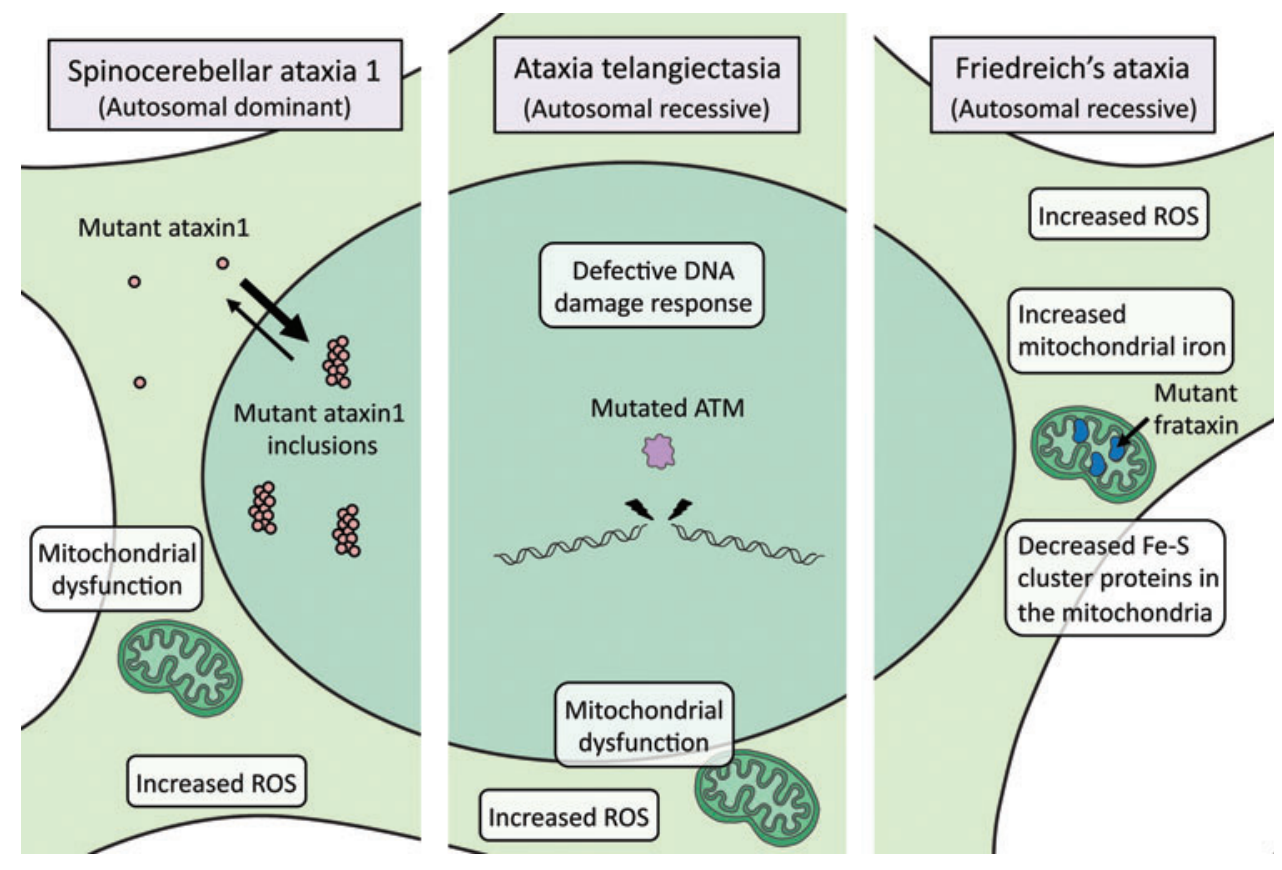

FIG. 14. Overview of the major forms of ataxia. In the autosomal dominant SCA1, expansion of polyglutamine repeats leads to aggregation of ataxin 1 , which can cause mitochondrial dysfunction and increased ROS levels. In ataxia telangiectasia, an autosomal recessive disorder caused by mutation in the ATM protein, which is involved in the DNA repair pathway, genomic instability occurs and oxidative stress responses are compromised, which cause increased ROS levels and mitochondrial dysfunction. In Friedrich's ataxia, another autosomal recessive disorder, mutations in the protein frataxin lead to defective Fe-S cluster protein biogenesis and elevated iron content. Similar to SCA1 and ataxia telengectasia, elevated ROS levels and mitochondrial malfunction are observed. ATM, ataxia-telangiectasia mutated. Color images are available online.

however, the first system, introduced by Harding, broadly classifies ataxias based on clinical and genetic criteria as those with a known metabolic disorder and those with unknown etiology (211). Later, as the causes of the ataxias have become clearer, additional classification criteria have been included and categorized into ataxias that are hereditary, nonhereditary, and acquired (272). The hereditary ataxias are further classified into autosomal dominant, autosomal recessive, and X-linked ataxias.

1. Autosomal dominant ataxias: spinocerebellar ataxia. Spinocerebellar ataxia 1 (SCA1) is the first ataxia with an identified genetic etiology (375). The disease is caused by expansion of CAG repeats in the coding region of ataxin-1 on the short arm of chromosome 6, which varies from 6 to 39 repeats in normal individuals and from 40 to 81 repeats in ataxia. The disease is autosomal dominant and results in degeneration of the cerebellum, spinal cord, and brainstem. Symptoms usually begin in the third or fourth decade of life with progressive deterioration in motor function and death 120 years after onset. In SCA1, degeneration of cerebellar Purkinje cells as well as certain brainstem nuclei occurs, leading to uncoordinated voluntary movement, impaired balance, and difficulty speaking and swallowing. The CAG repeats are unstable and prone to expansion, leading to the phenomenon of anticipation, where each successive generation has an earlier age of onset. Thus, there is a direct correlation between the size of the polyglutamine tract and the age of onset with juvenile cases of SCA1-bearing larger polyglutamine tracts in ataxin 1. Subsequently, other genetically transmitted ataxias have been identified, caused by mutations in other genes and named according to the order of discovery. CAG expansions have been identified in SCA2, MJD (Machado Joseph disease)/SCA3, SCA6, SCA7, and SCA17 in coding regions. The ataxias, SCA8, SCA10, SCA12, and SCA31, have repeat expansions in noncoding regions. Nonrepeat mutations also occur in ataxias such as mutations in beta-III spectrin (SPTBN2) in SCA5, tau tubulin kinase 2 in SCA11, a potassium channel in SCA13, PKC $\gamma$ in SCA14, inositol 1,4,5-triphosphate receptor type 1 in SCA15/ 16, fibroblast growth factor 14 in SCA27, and ATPase family gene 3 -like 2 in SCA28. All these mutations are inherited in an autosomal dominant manner.

2. Autosomal recessive ataxias: ataxia telangiectasia. Ataxia telangiectasia is characterized by an unsteady gait (ataxia) and dilation of blood vessels (telangiectases) in the conjunctiva of the eyes or facial skin (449). Positional cloning has localized the mutation to the ATM gene on chromosome 11, which encodes a polypeptide with a PI-3 kinase domain. The disease begins in childhood and is characterized by the degeneration or absence of the thymus and cerebellum and deficits in immune response leading to frequent pulmonary infections in patients. Other symptoms include marked sensitivity to ionizing radiation, premature aging, and chromosomal instability, as well as a predisposition to cancer and death within the second or third decade of life. At the molecular level, cells of telangiectasia patients 
undergo early senescence and have defects in DNA replication and cell cycle progression with extreme sensitivity to radiation. These cells exhibit cytoskeletal abnormalities and have a greater requirement for serum growth factors. Subsequent characterization of the gene product has revealed that ATM has sequence similarities to proteins in yeast, Drosophila, and mammals, which are involved in the detection of DNA damage and the control of cell cycle progression (450). ATM is a serine/threonine kinase that has several substrates and functions in addition to its role in DNA damage signaling (343). In response to DNA damage, induced by ionizing radiation, the multimeric inactive ATM undergoes autophosphorylation on Ser1981 to dissociate into an active monomeric form (22). ATM can also be activated in response to oxidative stress by forming dimers via a disulfide linkage between cysteine residues of monomers (195). Cells lacking ATM are hypersensitive to oxidative stress, and mice lacking ATM display oxidative injury in tissues with marked damage to the Purkinje cells of the cerebellum $(28,31)$. ATM-deleted cells have elevated levels of NADPH oxidase 4 (NOX4), which produces ROS such as $\mathrm{O}_{2}{ }^{--}$; knocking down NOX4 reduces DNA damage, DNA double-strand breaks, and replicative senescence (533). ATMdeficient cells have constitutively activated antioxidant defense pathways and display disturbed mitochondrial function, which could contribute to increased ROS levels (14). Structural organization of mitochondria is abnormal, and the mitochondrial membrane potential is decreased in these cells along with diminished respiratory capacity. Thus, oxidative stress and mitochondrial dysfunction play significant roles in cellular dysfunction in ataxia telangiectasia. Treating the mutant cells with the antioxidant, alpha lipoic acid, restores mitochondrial respiration rates. In addition, expression of wild-type ATM rescues impaired mitochondrial function in ATM-deficient cells (14). Targeting catalase to mitochondria alleviates symptoms in a mouse model of ataxia, which has been attributed to reduction of mitochondrial ROS (116). Targeting to mitochondria was first achieved by introducing the first 25 amino acids of the ornithine transcarbamylase leader sequence, added to the amino terminus of the catalase ORF (459). Mitochondrial catalase reduces the incidence of thymic lymphoma in $\mathrm{ATM}^{-1-}$ mice improving bone marrow hematopoiesis, macrophage differentiation, and partially reversing memory $\mathrm{T}$ cell developmental defects. Other therapies for ataxia telangiectasia include treatment with antioxidants such as NAC, which delays disease progression (420). Treating ATM mutant mice with the nitroxide antioxidant, tempol (4-hydroxy2,2,6,6-tetramethylpiperidine-N-oxyl), increased the life span of these mice by prolonging the latency to thymic lymphomas. Tempol treatment also decreased oxidative stress and associated damage (460).

3. Friedreich's ataxia. Similar to ataxia telangiectasia, Friedreich's ataxia (FRDA) is an autosomal recessive ataxia. The disorder affects the central and peripheral nervous systems as well as the heart. The genetic basis of FRDA includes expansion of GAA repeats or point mutations in the gene frataxin, which maps to chromosome 9 (81). A majority of mutations map to the first intron of frataxin with 8-22 expansions occurring in normal alleles. Some patients have an expansion of GAA repeats on one allele and point mutations on the other allele, whereas most have repeat expansions on both alleles. Patients with larger expansions typically have an earlier age of onset and additional complications and disease symptoms. There is a drastic reduction in the levels of frataxin in patients. (69). The onset of the disease usually occurs during adolescence, and symptoms include unsteady gait, the absence of lower limb reflexes, dysarthria, dysphagia, eye movement abnormalities, scoliosis, foot deformities, cardiomyopathy, and the presence of pyramidal signs (neural pathways controlling voluntary motor functions).

Frataxin is a low-molecular-weight protein of $23 \mathrm{kDa}$, which localizes to the mitochondria (277). Deletion of the frataxin homologue, $Y F H 1$, in yeast causes iron accumulation, loss of mtDNA, as well as sensitivity to $\mathrm{H}_{2} \mathrm{O}_{2}$ and iron $(21,159,535)$. In mice, knockout of the frataxin gene causes embryonic lethality, but some studies report no change in iron accumulation, while others observe elevated iron levels $(108,404)$. Decrease in the iron/sulfur (Fe-S) cluster-containing subunits of mitochondrial respiratory complexes I, II, and III has been described in endomyocardial biopsies of two FRDA patients, indicating a role of frataxin in $\mathrm{Fe}-\mathrm{S}$ protein homeostasis (437). In addition, activity of the Fe-S protein aconitase, which participates in iron homeostasis, is low in FRDA patients. The defects in iron metabolism and mitochondrial respiration have been attributed to elevated oxidative stress due to iron overload and its participation in the Fenton reaction to generate ROS. The role of iron in pathogenesis of FRDA has been debated, as it is not a consistent feature in FRDA cell culture and mouse models and has been considered to be a late event when Fe-S proteins are depleted $(34,108)$. However, more recently, Drosophila and CRISPR mouse models lacking frataxin have been shown to accumulate iron and toxicity $(86,87)$. Knockout of mouse frataxin in the central nervous system using AAV and CRISPR/Cas9 causes behavioral and neurological phenotypes similar to those reported earlier in a neuronal conditional knockout mouse model, including smaller body size, hunchback phenotype, impaired locomotion, and shortened life span (404). Both intracellular and extracellular accumulations of iron (both $\mathrm{Fe}^{2+}$ and $\mathrm{Fe}^{3+}$ ) are observed. Iron deposition has been found to trigger sphingolipid synthesis and activation of 3-phosphoinositide-dependent protein kinase-1 and myocyte enhancer factor-2 (Mef2). Mef2 then triggers transcription of downstream target genes, which mediate degeneration (87). Other studies have reported that frataxin depletion leads to elevated sensitivity to oxidative stress. Humanized transgenic mouse models of FRDA, Y87R, and Y47R harbor a human frataxin YAC with 190+90 GAA repeats on a mouse frataxin null background. These mice display impaired mitochondrial function and decreased GSH levels along with elevated oxidative stress, especially lipid peroxidation (3). Complex I activity is decreased, leading to increased compensatory activity of complex II, which generated higher levels of free radicals in the process. Both the mitochondrial and cytosolic compartments of cerebellar granule cells from these mice exhibit increased ROS production. Fibroblasts derived from FRDA patients show a blunted response to elevated iron levels. The $\mathrm{O}_{2}{ }^{\bullet-}$ detoxifying mitochondrial enzyme MnSOD fails to respond in FRDA fibroblasts exposed to iron. (250). This abnormality, which reflects depletion of $\mathrm{PGC} 1 \alpha$, a protein involved in mitochondrial energetics and the coactivator for PPAR $\gamma$ in FRDA cells, leads to suboptimal functioning of antioxidant defense pathways (332). In concordance with these 
findings, the hypersensitivity of fibroblasts from FRDA patients to oxidative stress can be prevented by reducing lipid peroxidation with deuterated PUFAs (currently under clinical trials) or by activating the Nrf2 pathway $(3,4)$.

Thus, it is clear from the above discussion that elevated oxidative stress contributes to the pathology of the various ataxias, which profoundly affect cerebellar function. Antioxidant therapy in these ataxias has been extensively attempted with varying efficacy, and detailed studies need to be conducted to arrive at more efficient drugs for these diseases (26).

\section{G. Aging and redox imbalance}

Aging, especially when coupled to redox imbalance, is a risk factor for cognitive and motor deficits as well as neurodegeneration. Age-dependent increase in oxidative damage to mtDNA has been reported, which can lead to impaired mitochondrial energetics and elevated oxidative stress (344). The amount of 8-OHdG, a DNA damage biomarker, increases progressively with normal aging in both nuclear DNA and mtDNA, with a much greater rate of accumulation in mtDNA. A 10 -fold increase in $8-\mathrm{OHdG}$ content in mtDNA compared with nuclear DNA has been observed in samples tested. In addition, in a cohort older than 70 years, a 15 -fold increase has been reported, further attesting to the vulnerability of mtDNA to damage. Aging has been associated with decline in the redox buffering capacity with decreases in GSH and cysteine levels $(141,417,475)$. In agreement with these studies, GSH levels are maintained in aged inbred albino Louvain (LOU/C) rats where cognitive functions are preserved (357). LOU rats are considered to be a model of healthy aging due to their increased life span without obesity and due to a low incidence of common age-related diseases (35). Aging involves altered or suboptimal response to environmental or physiological stress. Aged rats treated with menadione (vitamin $\mathrm{K}$ ), a redox cycling agent, are less responsive than younger rats. The antioxidant response is higher in younger than older rats. Supplementation of NAC is beneficial in aged rats reducing oxidative stress and improving mitochondrial function (102). There are numerous examples in literature where genes linked to maintenance of redox homeostasis are implicated in aging. In yeast, overexpression of Tsa1, an $\mathrm{H}_{2} \mathrm{O}_{2}$-scavenging enzyme, extends life span (208). Genetic disruption of Nrf2, the master regulator of antioxidant defense and redox balance, shortens the life span of female mice and also in males in other experimental models (556). Overexpression of glucose 6 phosphate dehydrogenase (G6PD), the rate-limiting step of the pentose phosphate pathway, increases life span in mice (369). G6PD is involved in the synthesis of nucleotide precursors and in the production of NADPH, which plays central roles in redox homeostasis in cells. G6PD-deficient mice display elevated oxidative stress and age-dependent neurodegeneration (246). Old G6PD-Tg male and female mice have decreased levels of 8-OHdG in the liver and brain with old females displaying reduced lipid peroxidation in the liver. In addition, the transgenic mice exhibit increased insulin sensitivity and decreased obesity as they age in comparison with their wildtype counterparts. Although some studies indicate that depletion or inhibition of proteins involved in antioxidant defense reduces life span, other studies report that this may not be the case. An analysis of deletion of 18 different genes participating in the antioxidant defense revealed a role for only the mice lacking Sodl, on life span (389). Interestingly, the $S o d l^{-1-}$ mice displayed elevated levels of thiol antioxidants such as GSH, thioredoxin, and sulfiredoxin. In several instances, transgenic mice overexpressing antioxidant enzymes such as catalase or SOD1 do not exhibit a longer life span $(228,389)$. As longevity is determined by multiple factors, which range from environmental factors to diet and stress response, caution should be exercised while interpreting the contributions of individual genes or gene products to life span. Some of the knockout mice used in the studies, for instance, the peroxiredoxin $1(\operatorname{Prdx} 1)$-deleted mice, have a shorter life span due to a higher incidence of cancer, rather than elevated oxidative stress.

During aging, the redox potential of the GSSG/GSH and Cys/Cys-SS couple also shifts to a more oxidized state (254, 445). Oxidation of the Cys/Cys-SS redox couple is a risk factor for cardiovascular disease and ocular disease. Antioxidant supplements prevent oxidation of cysteine/cystine in patients with age-related macular degeneration (354). In Hutchinson-Gilford progeria syndrome (HGPS), a disease characterized by premature aging, mitochondrial dysfunction and elevated oxidative stress are key molecular features (183, 425). HGPS is caused by constitutive expression of progerin, a truncated splicing mutant of the nuclear protein lamin A. Progerin exerts its effects in a dominant manner and affects mesenchymal stem cells (MSCs), underlying several phenotypes observed at the organismal level. Early death occurs, most frequently, due to myocardial infraction or stroke. iPSC-derived MSCs from HGPS patients are impaired in their ability to respond to oxidative stress and survive under hypoxic conditions. Dysregulation of the Nrf2 signaling pathway contributes to imbalanced redox control in HGPS cells (281). Progerin binds to Nrf2 and prevents its proper localization in the nucleus, which results in suboptimal Nrf2 transcriptional function and elevated oxidative stress (308). This pathway is also frequently disrupted in neurodegenerative diseases $(249,435,526)$. Thus, upregulation or stimulation of Nrf2 may have therapeutic potential.

In recent years, caloric restriction has gained appreciation as a means to increase longevity in several species, including worms, flies, yeast, and mammals. Redox signaling has been linked to dietary restriction. Caloric restriction triggers the antioxidant response program. This is not surprising, since during caloric restriction, cells undergo metabolic reprogramming to rely on OXPHOS for energy requirements more than glycolysis (310). Caloric restriction has been shown to elevate $\mathrm{H}_{2} \mathrm{~S}$, the gasotransmitter via the reverse transsulfuration pathway, plays pivotal roles in the process (218). Prior studies had shown that increased flux through the reverse transsulfuration pathway, which plays key roles in antioxidant defense, is a mediator of longevity $(257,513)$. Thus, upregulation of the reverse transsulfuration may have beneficial effects.

We had earlier proposed that $\mathrm{H}_{2} \mathrm{~S}$-mediated sulfhydration/ persulfidation can protect proteins from irreversible oxidation on cysteine residues (382). The $\mathrm{CySSO}_{2} \mathrm{H}$ (perthiosulfinic) and $\mathrm{CySSO}_{3} \mathrm{H}$ (perthiosulfonic) oxidation products of persulfides can be recycled by the reduction of their S-S moieties, which does not occur in the case of the $\mathrm{CySO}_{3} \mathrm{H}$ oxidation product of unmodified (unsulfhydrated) cysteine 
residues on proteins (386). Our hypothesis of protection of SH groups of cysteine residues by persulfidation has been verified in the case of phosphatase and tensin homologue deleted on chromosome 10, PTEN, and GAPDH $(169,372)$. Thus, $\mathrm{H}_{2} \mathrm{~S}$ production and sulfhydration could prevent oxidation of proteins and contribute to longevity, both at the level of protein half-life and overall life span. Consistent with the contribution of protein integrity to life span, in the longliving naked mole-rats, age-related accumulation of oxidation damage to thiol groups and age-associated upregulation of homeostatic proteolytic activity are significantly attenuated compared with mice (390). A point to be noted is that although young mole-rats have greater protein oxidation compared with young mice, the increase in oxidative damage over time (two decades) is minimal, indicating efficient protein homeostatic mechanisms.

\section{Concluding Remarks}

Fluctuations in redox state are an integral part of cellular physiology. Subtoxic levels of free radicals have roles in several signaling events. For instance, ROS are necessary for differentiation of certain cell types (448). It is only when the oxidant levels cross a "threshold" that damage to cellular components occurs, which, if not corrected by endogenous antifree radical machinery, causes irreversible damage and cell death. Thus, the concept of "oxidative eustress" has been proposed to distinguish it from "oxidative distress," which occurs under pathological conditions and involves higher levels of oxidants $(447,472,473)$. Thus, there are different metabolic set points and thresholds that are dynamic in nature. Accordingly, situations arise where elevated levels of free radicals as well as upregulated antioxidant defense can occur leading to altered metabolic set points, which could still maintain cellular functions. In normal conditions, a slight upregulation of antioxidant defense mechanisms can counter the redox imbalance created by metabolic processes. However, under pathogenic conditions or during stress, the redox balance may be tilted resulting in increased free radical generation (Fig. 15). When cells cannot counteract this stress, cell death ensues. It is also becoming increasingly clear that oxidative stress has adverse effects in neurodegeneration, while in cancers, the opposite is true. Mitigating oxidative stress in cancer may be detrimental, as cancer cells have been reprogrammed to elevate their antioxidant defenses to maximize cell growth and proliferation. In this case, inducing oxidative stress may be beneficial. In cancers, there is over proliferation and growth, whereas in neurodegeneration, there is atrophy and loss of neuronal cells. Thus, depending on the context, modulating the redox milieu of cells can have different effects.

Oxidative stress plays a significant role in disease progression and neurodegeneration. Oxidative stress, mitochondrial dysfunction, and proteostasis are intimately linked, with mitochondrial malfunction leading to elevated oxidative stress and vice versa. Thus, ameliorating oxidative stress and/ or stimulating redox signaling pathways responsible for corrective responses may prevent or delay neurodegeneration. As the redox regulatory network in cells is vast, with interconnecting nodes, disruption of a major regulatory point may lead to a cascade of events that culminate in aberrant stress response. When this process remains uncorrected, as in

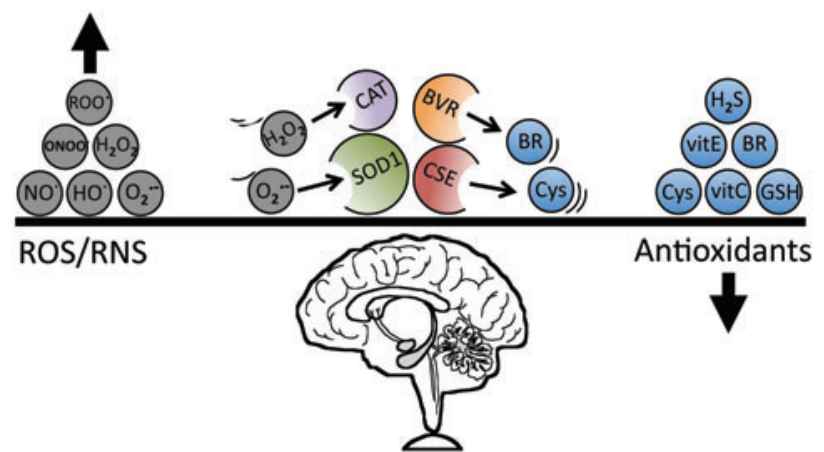

FIG. 15. Dysregulated redox homeostasis in neurodegeneration. Most neurodegenerative disorders exhibit oxidative stress and mitochondrial malfunction, leading to elevated production of ROS and RNS. In most cases, there is a decrease in endogenous antioxidants such as glutathione (GSH), cysteine (Cys), bilirubin (BR), vitamin E (VitE), and vitamin $\mathrm{C}$ (Vit $\mathrm{C}$ ). Under normal conditions, antioxidant enzymes such as CSE (which produces cysteine and hydrogen sulfide, $\mathrm{H}_{2} \mathrm{~S}$ ), catalase/CAT (which detoxifies $\mathrm{H}_{2} \mathrm{O}_{2}$ ), SOD1 (dismutates superoxide to $\mathrm{H}_{2} \mathrm{O}_{2}$ ), and BVR (that produces the lipophilic antioxidant bilirubin/BR) and other enzymes neutralize or scavenge the free radicals to maintain redox balance. During neurodegeneration, the activity and/ or expression of one or more of these cytoprotective enzymes are affected leading to neurotoxicity. BVR, biliverdin reductase. Color images are available online.

the case of neurodegeneration, cell death ensues. Another cellular process linked to oxidative stress is autophagy, the process by which damaged proteins and organelles are cleared (41). Autophagy and oxidative stress have been increasingly interlinked in several studies, although it is still unclear which species of ROS or RNS initiates the process $(88,149,561)$. Mitochondrial functions are also intimately associated with the process as they are the predominant generators of ROS in cells (297). Nevertheless, excess oxidative stress can compromise this process too, leading to buildup of toxic aggregates, which also contribute to redox imbalance. Signaling pathways operating in the lysosomes, the organelles playing central roles in autophagy have also been linked to redox regulation (565). Impaired autophagy has been observed in several different neurodegenerative disorders and promoting clearance of unfolded or aggregated proteins and dysfunctional organelles may afford neuroprotection. More recently, the Nrf2 pathway, recognized as an antioxidant pathway, has also been shown to modulate autophagic processes via p62, an autophagic receptor, indicating that a fine balance between redox signaling in mitigating excess oxidative stress while maintaining normal cellular processes is essential for optimal cell function $(74,274)$. Thus, it is not surprising that use of antioxidants to counter neurodegeneration can impair essential processes such as autophagy in certain instances (300).

Thus, identification of the origins of neurodegenerative processes in various diseases becomes critical. Although a plethora of studies have been conducted on neurodegenerative diseases, the differential susceptibility of specific regions of the brain in different neurodegenerative diseases is intriguing. Thus, the molecular basis of SNV is still elusive. A combination of genetic and epigenetic factors, environmental 
interactions, and nature of the mutation contribute to the initiation and progression of neurodegeneration. Neurodegenerative diseases such as AD, PD, and ALS can arise due to genetic causes (familial AD, PD, or ALS) or arise sporadically. $\mathrm{HD}$ on the contrary is the only neurodegenerative disease where all cases have mutations in the huntingtin gene. A stressor threshold model has been proposed in which the intrinsic susceptibilities of neuronal cell types to stressors and specific disease-related misfolding proteins determine neuronal toxicity. Specific combinations of genetic predispositions and environmental stressors elicit age-dependent stress and proteostasis dysfunction in vulnerable neurons (451). A common feature of diverse neurodegenerative diseases is mitochondrial dysfunction and oxidative stress, which are intimately linked (305). Whether oxidative stress elicits mitochondrial dysfunction or vice versa is a matter of debate.

Although it is becoming increasingly clear that oxidative stress is associated with several diseases, including neurodegenerative disorders, antioxidant supplementation has had limited therapeutic benefit in terms of "curing" disease, a phenomenon termed the "antioxidant paradox" (203, 206). Multiple reasons have been postulated to account for failure of antioxidants to reverse or halt disease progression $(204,205)$. In several instances, administration of antioxidants does not reduce oxidative stress significantly. The time and stage at which the antioxidant is to be delivered are still poorly researched. Some situations, where there is a paucity of the antioxidant or molecule under investigation, may benefit. For example, the supplementation of cysteine and GSH has proved beneficial in several age-related conditions. These interventions too depend on the bioavailability of the compound being administered. The studies on mouse models of disease do not translate to human trials due to differences in intrinsic antioxidant networks. For instance, vitamin E was reported to be beneficial for a mouse model of familial ALS, whereas this was not the case in ALS patients (196, 376). Despite these limitations, more recently, edavarone (radicava), an antioxidant that eliminates lipid peroxides and $\bullet \mathrm{OH}$, has been found to be effective in ALS patients and approved for use $(436,543)$. Another pitfall of utilizing certain antioxidants (e.g., polyphenols and ascorbate) is that they could interact with transition metals, such as iron and copper, and mediate pro-oxidant effects. Antioxidants have different targets in cells, both in terms of scavenging or neutralizing free radicals directly or by modulation of redox regulatory networks. Thus, an antioxidant may counter the activity of one type of ROS but leave another intact. In addition, as discussed earlier, certain thiols such as cysteine and GSH act via post-translational modifications, which can account for their activity in cells. These activities are very often confused with "antioxidant" effects. Very often, the supplementation of the depleted antioxidant molecule, by itself, does not provide a robust protective effect. Targeting the antioxidant to specific sites may also alter outcomes in clinical trials. Upregulating the biosynthetic pathway responsible for production of the molecule may be more effective. Understanding the redox state of the cell may help design better therapeutics.

In several instances, a reversible oxidized redox state precedes free radical damage and can be targeted for the treatment of neurodegenerative diseases. Priming cells with mild forms of stress may induce cytoprotective pathways that convey resistance to future insults. Thus, the concept of "hormesis" or adaptive response has been proposed (65-67, 349). This is similar in concept to the process of vaccination, where a low or attenuated dose of the pathogen can trigger host responses that afford future protection. Hormetic mechanisms are part of the normal physiology of the brain, and their disruption may be detrimental to neuronal and glial systems. Intermittent fasting, regular exercise, and consumption of dietary phytochemicals can promote adaptive cellular stress response pathways that protect against and counteract several diseases $(339,410)$. Low levels of stressor molecules can induce pathways that maintain redox balance, such as the Nrf2 pathway and the PPAR $\gamma$ pathway. Another target is the reverse transsulfuration pathway, which is frequently disrupted in neurodegeneration. This pathway is central to the maintenance of redox balance via the synthesis of GSH and cysteine, major antioxidant cytoprotectants in cells $(381,453)$. In addition, this pathway is also responsible for the generation of $\mathrm{H}_{2} \mathrm{~S}$ by the enzymes CSE and CBS. Recently, it was shown that $\mathrm{H}_{2} \mathrm{~S}$ can also modulate Nrf2mediated antioxidant signaling (549). The newly emerging area of gasotransmitter signaling, encompassing $\mathrm{NO}, \mathrm{CO}$, and $\mathrm{H}_{2} \mathrm{~S}$, adds several layers of complexity to the intricate network of redox signaling. The interplay of various redox active molecules is yet to be elucidated. Especially relevant is the interplay between oxygen and $\mathrm{H}_{2} \mathrm{~S}$ signaling, which plays fundamental roles in cerebrovascular function. Another area of interest is the overlap of gasotransmitter signaling with the microbiota of the host, which could play important roles in redox regulation as well as modulation of the gut/brain axis.

In addition, identification of sensors of various forms of stress may reveal additional hubs and targets of redox regulation. Oxidative stress and ER stress can reprogram cells to adapt for survival. Sensor functions present on organelles such as the Golgi and lysosomes may also play significant roles in maintenance of redox homeostasis whose disruption could mediate neurodegeneration $(322,452)$. The Golgi is emerging as a major stress response organelle, similar to the ER. It has been proposed that changes in the structure and organization of the Golgi apparatus mediate sensing of stress stimuli well before cell death and neurodegeneration. Fragmentation of the Golgi has been observed in various neurodegenerative diseases such as AD, ALS, and HD (182, 255, 452). The spatiotemporal control of gene regulatory networks that govern the cytoprotective stress responses of an organism is especially important. Transcription factors that regulate major hubs of redox control may serve as targets of drug design and therapeutics. With the advent of high-throughput methodologies and platforms, the integration of genomics, epigenomics, proteomics, and biochemical analyses, metabolomics, and identification of signaling networks should pave the way for personalized medicine. These developments, coupled with nutritional and environmental analysis, portend a promising future for redox medicine.

\section{Acknowledgment}

This work was supported by USPHS grants DA000266 and MH18501 to S.H.S.

\section{References}

1. A novel gene containing a trinucleotide repeat that is expanded and unstable on Huntington's disease 
chromosomes. The Huntington's Disease Collaborative Research Group. Cell 72: 971-983, 1993.

2. Abe $\mathrm{K}$ and Kimura $\mathrm{H}$. The possible role of hydrogen sulfide as an endogenous neuromodulator. J Neurosci 16: 1066-1071, 1996.

3. Abeti R, Parkinson MH, Hargreaves IP, Angelova PR, Sandi C, Pook MA, Giunti P, and Abramov AY. Mitochondrial energy imbalance and lipid peroxidation cause cell death in Friedreich's ataxia. Cell Death Dis 7: e2237, 2016.

4. Abeti R, Uzun E, Renganathan I, Honda T, Pook MA, and Giunti P. Targeting lipid peroxidation and mitochondrial imbalance in Friedreich's ataxia. Pharmacol Res 99: 344350, 2015.

5. Abiko Y, Shinkai Y, Unoki T, Hirose R, Uehara T, and Kumagai Y. Polysulfide $\mathrm{Na}_{2} \mathrm{~S}_{4}$ regulates the activation of PTEN/Akt/CREB signaling and cytotoxicity mediated by 1,4-naphthoquinone through formation of sulfur adducts. Sci Rep 7: 4814, 2017.

6. Abo M and Weerapana E. Chemical probes for redox signaling and oxidative stress. Antioxid Redox Signal 2017; [Eup ahead of print]; DOI 10.1089/ars.2017.7408.

7. Acevedo-Torres K, Berrios L, Rosario N, Dufault V, Skatchkov S, Eaton MJ, Torres-Ramos CA, and AyalaTorres S. Mitochondrial DNA damage is a hallmark of chemically induced and the R6/2 transgenic model of Huntington's disease. DNA Repair (Amst) 8: 126-136, 2009.

8. Acuna AI, Esparza M, Kramm C, Beltran FA, Parra AV, Cepeda C, Toro CA, Vidal RL, Hetz C, Concha II, Brauchi S, Levine MS, and Castro MA. A failure in energy metabolism and antioxidant uptake precede symptoms of Huntington's disease in mice. Nat Commun 4: 2917, 2013.

9. Adelman R, Saul RL, and Ames BN. Oxidative damage to DNA: relation to species metabolic rate and life span. Proc Natl Acad Sci U S A 85: 2706-2708, 1988.

10. Alfonso-Prieto M, Biarnes X, Vidossich P, and Rovira C. The molecular mechanism of the catalase reaction. $J \mathrm{Am}$ Chem Soc 131: 11751-11761, 2009.

11. Alin P, Danielson UH, and Mannervik B. 4-Hydroxyalk2-enals are substrates for glutathione transferase. FEBS Lett 179: 267-270, 1985.

12. Alvarez-Erviti L, Couch Y, Richardson J, Cooper JM, and Wood MJ. Alpha-synuclein release by neurons activates the inflammatory response in a microglial cell line. $\mathrm{Neu}$ rosci Res 69: 337-342, 2011.

13. Amar F, Sherman MA, Rush T, Larson M, Boyle G, Chang L, Gotz J, Buisson A, and Lesne SE. The amyloid$\beta$ oligomer $\mathrm{A} \beta * 56$ induces specific alterations in neuronal signaling that lead to tau phosphorylation and aggregation. Sci Signal 10: eaal 2021, 2017.

14. Ambrose M, Goldstine JV, and Gatti RA. Intrinsic mitochondrial dysfunction in ATM-deficient lymphoblastoid cells. Hum Mol Genet 16: 2154-2164, 2007.

15. Aoyama K, Suh SW, Hamby AM, Liu J, Chan WY, Chen $\mathrm{Y}$, and Swanson RA. Neuronal glutathione deficiency and age-dependent neurodegeneration in the EAAC1 deficient mouse. Nat Neurosci 9: 119-126, 2006.

16. Atherton JF, McIver EL, Mullen MR, Wokosin DL, Surmeier DJ, and Bevan MD. Early dysfunction and progressive degeneration of the subthalamic nucleus in mouse models of Huntington's disease. Elife 5: e21616, 2016.
17. Atwal RS, Desmond CR, Caron N, Maiuri T, Xia J, Sipione S, and Truant R. Kinase inhibitors modulate huntingtin cell localization and toxicity. Nat Chem Biol 7: 453-460, 2011.

18. Atwal RS, Xia J, Pinchev D, Taylor J, Epand RM, and Truant R. Huntingtin has a membrane association signal that can modulate huntingtin aggregation, nuclear entry and toxicity. Hum Mol Genet 16: 2600-2615, 2007.

19. Ayala A, Munoz MF, and Arguelles S. Lipid peroxidation: production, metabolism, and signaling mechanisms of malondialdehyde and 4-hydroxy-2-nonenal. Oxid Med Cell Longev 2014: 360438, 2014.

20. Ayton S, Lei P, Duce JA, Wong BX, Sedjahtera A, Adlard PA, Bush AI, and Finkelstein DI. Ceruloplasmin dysfunction and therapeutic potential for Parkinson disease. Ann Neurol 73: 554-559, 2013.

21. Babcock M, de Silva D, Oaks R, Davis-Kaplan S, Jiralerspong S, Montermini L, Pandolfo M, and Kaplan J. Regulation of mitochondrial iron accumulation by Yfh1p, a putative homolog of frataxin. Science 276: 1709-1712, 1997.

22. Bakkenist CJ and Kastan MB. DNA damage activates ATM through intermolecular autophosphorylation and dimer dissociation. Nature 421: 499-506, 2003.

23. Bala KA, Dogan M, Mutluer T, Kaba S, Aslan O, Balahoroglu R, Cokluk E, Ustyol L, and Kocaman S. Plasma amino acid profile in autism spectrum disorder (ASD). Eur Rev Med Pharmacol Sci 20: 923-929, 2016.

24. Balogh LM and Atkins WM. Interactions of glutathione transferases with 4-hydroxynonenal. Drug Metab Rev 43: 165-178, 2011.

25. Bame M, Grier RE, Needleman R, and Brusilow WS. Amino acids as biomarkers in the SOD1(G93A) mouse model of ALS. Biochim Biophys Acta 1842: 79-87, 2014.

26. Barca E, Emmanuele V, DiMauro S, Toscano A, and Quinzii CM. Anti-oxidant drugs: novelties and clinical implications in cerebellar ataxias. Curr Neuropharmacol 17: 21-32, 2019.

27. Barcia C, Ros CM, Carrillo MA, Ros F, Gomez A, de Pablos V, Bautista-Hernandez V, Sanchez-Bahillo A, Villalba EF, and Herrero MT. Increase of secondary processes of microglial and astroglial cells after MPTPinduced degeneration in substantia nigra pars compacta of non human primates. J Neural Transm Suppl 253-258, 2009.

28. Barlow C, Dennery PA, Shigenaga MK, Smith MA, Morrow JD, Roberts LJ, 2nd, Wynshaw-Boris A, and Levine RL. Loss of the Ataxia-telangiectasia gene product causes oxidative damage in target organs. Proc Natl Acad Sci U S A 96: 9915-9919, 1999.

29. Bartesaghi S and Radi R. Fundamentals on the biochemistry of peroxynitrite and protein tyrosine nitration. Redox Biol 14: 618-625, 2018.

30. Bartzokis G, Cummings J, Perlman S, Hance DB, and Mintz J. Increased basal ganglia iron levels in Huntington disease. Arch Neurol 56: 569-574, 1999.

31. Barzilai A, Rotman G, and Shiloh Y. ATM deficiency and oxidative stress: a new dimension of defective response to DNA damage. DNA Repair (Amst) 1: 3-25, 2002.

32. Baumann K, Mandelkow EM, Biernat J, Piwnica-Worms $\mathrm{H}$, and Mandelkow E. Abnormal Alzheimer-like phosphorylation of tau-protein by cyclin-dependent kinases cdk2 and cdk5. FEBS Lett 336: 417-424, 1993. 
33. Baxter AJ, Brugha TS, Erskine HE, Scheurer RW, Vos T, and Scott JG. The epidemiology and global burden of autism spectrum disorders. Psychol Med 45: 601-613, 2015.

34. Bayot A, Santos R, Camadro JM, and Rustin P. Friedreich's ataxia: the vicious circle hypothesis revisited. BMC Med 9: 112, 2011.

35. Bazin H, Kints JP, and Rousseaux J. Genetic control of the resistance to spontaneous immunocytoma (plasmacytomaIR tumour) development in LOU/C rats. Anticancer Res 6: 45-48, 1986.

36. Behnia F, Parets SE, Kechichian T, Yin H, Dutta EH, Saade GR, Smith AK, and Menon R. Fetal DNA methylation of autism spectrum disorders candidate genes: association with spontaneous preterm birth. Am J Obstet Gynecol 212: 533.e1-533.e9, 2015.

37. Belarbi K, Cuvelier E, Destee A, Gressier B, and ChartierHarlin MC. NADPH oxidases in Parkinson's disease: a systematic review. Mol Neurodegener 12: 84, 2017.

38. Beltran FA, Acuna AI, Miro MP, Angulo C, Concha II, and Castro MA. Ascorbic acid-dependent GLUT3 inhibition is a critical step for switching neuronal metabolism. J Cell Physiol 226: 3286-3294, 2011.

39. Belzil VV, Bauer PO, Prudencio M, Gendron TF, Stetler CT, Yan IK, Pregent L, Daughrity L, Baker MC, Rademakers R, Boylan K, Patel TC, Dickson DW, and Petrucelli L. Reduced C9orf72 gene expression in c9FTD/ALS is caused by histone trimethylation, an epigenetic event detectable in blood. Acta Neuropathol 126: 895-905, 2013.

40. Benchoua A, Trioulier Y, Zala D, Gaillard MC, Lefort N, Dufour N, Saudou F, Elalouf JM, Hirsch E, Hantraye P, Deglon N, and Brouillet E. Involvement of mitochondrial complex II defects in neuronal death produced by Nterminus fragment of mutated huntingtin. Mol Biol Cell 17: 1652-1663, 2006.

41. Bento CF, Renna M, Ghislat G, Puri C, Ashkenazi A, Vicinanza M, Menzies FM, and Rubinsztein DC. Mammalian autophagy: how does it work? Annu Rev Biochem 85: 685-713, 2016.

42. Beretta S, Sala G, Mattavelli L, Ceresa C, Casciati A, Ferri A, Carri MT, and Ferrarese C. Mitochondrial dysfunction due to mutant copper/zinc superoxide dismutase associated with amyotrophic lateral sclerosis is reversed by N-acetylcysteine. Neurobiol Dis 13: 213-221, 2003.

43. Berggren KL, Chen J, Fox J, Miller J, Dodds L, Dugas B, Vargas L, Lothian A, McAllum E, Volitakis I, Roberts B, Bush AI, and Fox JH. Neonatal iron supplementation potentiates oxidative stress, energetic dysfunction and neurodegeneration in the R6/2 mouse model of Huntington's disease. Redox Biol 4: 363-374, 2015.

44. Berneburg M, Grether-Beck S, Kurten V, Ruzicka T, Briviba K, Sies H, and Krutmann J. Singlet oxygen mediates the UVA-induced generation of the photoagingassociated mitochondrial common deletion. J Biol Chem 274: 15345-15349, 1999.

45. Berry CE and Hare JM. Xanthine oxidoreductase and cardiovascular disease: molecular mechanisms and pathophysiological implications. J Physiol 555: 589-606, 2004.

46. Bian K and Murad F. Nitric oxide (NO)_biogeneration, regulation, and relevance to human diseases. Front Biosci 8: d264-d278, 2003.

47. Biteau B, Labarre J, and Toledano MB. ATP-dependent reduction of cysteine-sulphinic acid by S. cerevisiae sulphiredoxin. Nature 425: 980-984, 2003.
48. Blesa J, Trigo-Damas I, Quiroga-Varela A, and JacksonLewis VR. Oxidative stress and Parkinson's disease. Front Neuroanat 9: 91, 2015.

49. Borghi R, Patriarca S, Traverso N, Piccini A, Storace D, Garuti A, Gabriella C, Patrizio O, and Massimo T. The increased activity of BACE1 correlates with oxidative stress in Alzheimer's disease. Neurobiol Aging 28: 10091014, 2007.

50. Boveris A and Cadenas E. Mitochondrial production of superoxide anions and its relationship to the antimycin insensitive respiration. FEBS Lett 54: 311-314, 1975.

51. Braak H, Del Tredici K, Rub U, de Vos RA, Jansen Steur EN, and Braak E. Staging of brain pathology related to sporadic Parkinson's disease. Neurobiol Aging 24: 197211, 2003.

52. Bradley-Whitman MA, Timmons MD, Beckett TL, Murphy MP, Lynn BC, and Lovell MA. Nucleic acid oxidation: an early feature of Alzheimer's disease. J Neurochem 128: 294-304, 2014.

53. Brand MD, Affourtit C, Esteves TC, Green K, Lambert AJ, Miwa S, Pakay JL, and Parker N. Mitochondrial superoxide: production, biological effects, and activation of uncoupling proteins. Free Radic Biol Med 37: 755-767, 2004.

54. Brennan WA, Jr., Bird ED, and Aprille JR. Regional mitochondrial respiratory activity in Huntington's disease brain. J Neurochem 44: 1948-1950, 1985.

55. Brigelius-Flohe R and Maiorino M. Glutathione peroxidases. Biochim Biophys Acta 1830: 3289-3303, 2013.

56. Briggs RG and Fee JA. Sulfhydryl reactivity of human erythrocyte superoxide dismutase. On the origin of the unusual spectral properties of the protein when prepared by a procedure utilizing chloroform and ethanol for the precipitation of hemoglobin. Biochim Biophys Acta 537: 100-109, 1978.

57. Brouillet E, Jacquard C, Bizat N, and Blum D. 3Nitropropionic acid: a mitochondrial toxin to uncover physiopathological mechanisms underlying striatal degeneration in Huntington's disease. J Neurochem 95: 1521-1540, 2005.

58. Browne SE, Bowling AC, MacGarvey U, Baik MJ, Berger SC, Muqit MM, Bird ED, and Beal MF. Oxidative damage and metabolic dysfunction in Huntington's disease: selective vulnerability of the basal ganglia. Ann Neurol 41: 646-653, 1997.

59. Browne SE, Ferrante RJ, and Beal MF. Oxidative stress in Huntington's disease. Brain Pathol 9: 147-163, 1999.

60. Butterfield DA and Boyd-Kimball D. Amyloid betapeptide(1-42) contributes to the oxidative stress and neurodegeneration found in Alzheimer disease brain. Brain Pathol 14: 426-432, 2004.

61. Cadet J, Davies KJA, Medeiros MH, Di Mascio P, and Wagner JR. Formation and repair of oxidatively generated damage in cellular DNA. Free Radic Biol Med 107: 1334, 2017.

62. Cadet J, Ravanat JL, Martinez GR, Medeiros MH, and Di Mascio P. Singlet oxygen oxidation of isolated and cellular DNA: product formation and mechanistic insights. Photochem Photobiol 82: 1219-1225, 2006.

63. Cadet $\mathrm{J}$ and Wagner JR. Oxidatively generated base damage to cellular DNA by hydroxyl radical and oneelectron oxidants: similarities and differences. Arch Biochem Biophys 557: 47-54, 2014. 
64. Cai H, Wang Y, McCarthy D, Wen H, Borchelt DR, Price DL, and Wong PC. BACE1 is the major beta-secretase for generation of Abeta peptides by neurons. Nat Neurosci 4: 233-234, 2001.

65. Calabrese EJ. Preconditioning is hormesis part I: documentation, dose-response features and mechanistic foundations. Pharmacol Res 110: 242-264, 2016.

66. Calabrese EJ. Preconditioning is hormesis part II: how the conditioning dose mediates protection: dose optimization within temporal and mechanistic frameworks. Pharmacol Res 110: 265-275, 2016.

67. Calabrese EJ and Mattson MP. How does hormesis impact biology, toxicology, and medicine? NPJ Aging Mech Dis 3: 13, 2017.

68. Calabrese L, Federici G, Bannister WH, Bannister JV, Rotilio G, and Finazzi-Agro A. Labile sulfur in human superoxide dismutase. Eur J Biochem 56: 305-309, 1975.

69. Campuzano V, Montermini L, Molto MD, Pianese L, Cossee M, Cavalcanti F, Monros E, Rodius F, Duclos F, Monticelli A, Zara F, Canizares J, Koutnikova H, Bidichandani SI, Gellera C, Brice A, Trouillas P, De Michele G, Filla A, De Frutos R, Palau F, Patel PI, Di Donato S, Mandel JL, Cocozza S, Koenig M, and Pandolfo M. Friedreich's ataxia: autosomal recessive disease caused by an intronic GAA triplet repeat expansion. Science 271: 1423-1427, 1996.

70. Cappelletti G, Surrey T, and Maci R. The parkinsonism producing neurotoxin $\mathrm{MPP}^{+}$affects microtubule dynamics by acting as a destabilising factor. FEBS Lett 579: 47814786, 2005.

71. Caraveo G, Auluck PK, Whitesell L, Chung CY, Baru V, Mosharov EV, Yan X, Ben-Johny M, Soste M, Picotti P, Kim H, Caldwell KA, Caldwell GA, Sulzer D, Yue DT, and Lindquist $\mathrm{S}$. Calcineurin determines toxic versus beneficial responses to alpha-synuclein. Proc Natl Acad Sci U S A 111: E3544-E3552, 2014.

72. Carlsson LM, Jonsson J, Edlund T, and Marklund SL. Mice lacking extracellular superoxide dismutase are more sensitive to hyperoxia. Proc Natl Acad Sci U S A 92: 6264-6268, 1995.

73. Carocho $\mathrm{M}$ and Ferreira IC. A review on antioxidants, prooxidants and related controversy: natural and synthetic compounds, screening and analysis methodologies and future perspectives. Food Chem Toxicol 51: 15-25, 2013.

74. Carroll B, Otten EG, Manni D, Stefanatos R, Menzies FM, Smith GR, Jurk D, Kenneth N, Wilkinson S, Passos JF, Attems J, Veal EA, Teyssou E, Seilhean D, Millecamps S, Eskelinen EL, Bronowska AK, Rubinsztein DC, Sanz A, and Korolchuk VI. Oxidation of SQSTM1/p62 mediates the link between redox state and protein homeostasis. Nat Commun 9: 256, 2018.

75. Carroll J, Fearnley IM, Skehel JM, Shannon RJ, Hirst J, and Walker JE. Bovine complex I is a complex of 45 different subunits. J Biol Chem 281: 32724-32727, 2006.

76. Casareno RL, Waggoner D, and Gitlin JD. The copper chaperone CCS directly interacts with copper/zinc superoxide dismutase. J Biol Chem 273: 23625-23628, 1998.

77. Castano A, Herrera AJ, Cano J, and Machado A. Lipopolysaccharide intranigral injection induces inflammatory reaction and damage in nigrostriatal dopaminergic system. J Neurochem 70: 1584-1592, 1998.

78. Castro M, Caprile T, Astuya A, Millan C, Reinicke K, Vera JC, Vasquez O, Aguayo LG, and Nualart F. High-affinity sodium-vitamin C co-transporters (SVCT) expression in embryonic mouse neurons. J Neurochem 78: 815-823, 2001.
79. Castro MA, Angulo C, Brauchi S, Nualart F, and Concha II. Ascorbic acid participates in a general mechanism for concerted glucose transport inhibition and lactate transport stimulation. Pflug Arch 457: 519-528, 2008.

80. Castro MA, Beltran FA, Brauchi S, and Concha II. A metabolic switch in brain: glucose and lactate metabolism modulation by ascorbic acid. J Neurochem 110: 423-440, 2009.

81. Chamberlain S, Shaw J, Rowland A, Wallis J, South S, Nakamura Y, von Gabain A, Farrall M, and Williamson R. Mapping of mutation causing Friedreich's ataxia to human chromosome 9. Nature 334: 248-250, 1988.

82. Chan CS, Guzman JN, Ilijic E, Mercer JN, Rick C, Tkatch T, Meredith GE, and Surmeier DJ. "Rejuvenation" protects neurons in mouse models of Parkinson's disease. Nature 447: 1081-1086, 2007.

83. Chauhan A and Chauhan V. Oxidative stress in autism. Pathophysiology 13: 171-181, 2006.

84. Chauhan A, Gu F, Essa MM, Wegiel J, Kaur K, Brown WT, and Chauhan V. Brain region-specific deficit in mitochondrial electron transport chain complexes in children with autism. J Neurochem 117: 209-220, 2011.

85. Chen CM, Wu YR, Cheng ML, Liu JL, Lee YM, Lee PW, Soong BW, and Chiu DT. Increased oxidative damage and mitochondrial abnormalities in the peripheral blood of Huntington's disease patients. Biochem Biophys Res Commun 359: 335-340, 2007.

86. Chen K, Ho TS, Lin G, Tan KL, Rasband MN, and Bellen HJ. Loss of frataxin activates the iron/sphingolipid/PDK1/ Mef2 pathway in mammals. Elife 5: e20732, 2016.

87. Chen K, Lin G, Haelterman NA, Ho TS, Li T, Li Z, Duraine L, Graham BH, Jaiswal M, Yamamoto S, Rasband $\mathrm{MN}$, and Bellen HJ. Loss of frataxin induces iron toxicity, sphingolipid synthesis, and Pdk1/Mef2 activation, leading to neurodegeneration. Elife 5: pii: e16043, 2016.

88. Chen Y, Azad MB, and Gibson SB. Superoxide is the major reactive oxygen species regulating autophagy. Cell Death Differ 16: 1040-1052, 2009.

89. Chen YI, Wei PC, Hsu JL, Su FY, and Lee WH. NPGPx (GPx7): a novel oxidative stress sensor/transmitter with multiple roles in redox homeostasis. Am J Transl Res 8: 1626-1640, 2016

90. Chew J, Gendron TF, Prudencio M, Sasaguri H, Zhang YJ, Castanedes-Casey M, Lee CW, Jansen-West K, Kurti A, Murray ME, Bieniek KF, Bauer PO, Whitelaw EC, Rousseau L, Stankowski JN, Stetler C, Daughrity LM, Perkerson EA, Desaro P, Johnston A, Overstreet K, Edbauer D, Rademakers R, Boylan KB, Dickson DW, Fryer JD, and Petrucelli L. Neurodegeneration. C9ORF72 repeat expansions in mice cause TDP-43 pathology, neuronal loss, and behavioral deficits. Science 348: 1151-1154, 2015.

91. Chez MG, Buchanan CP, Aimonovitch MC, Becker M, Schaefer K, Black C, and Komen J. Double-blind, placebo-controlled study of L-carnosine supplementation in children with autistic spectrum disorders. $J$ Child Neurol 17: 833-837, 2002.

92. Chiba K, Trevor A, and Castagnoli N, Jr. Metabolism of the neurotoxic tertiary amine, MPTP, by brain monoamine oxidase. Biochem Biophys Res Commun 120: 574-578, 1984.

93. Cho DH, Nakamura T, Fang J, Cieplak P, Godzik A, Gu Z, and Lipton SA. S-nitrosylation of Drp1 mediates betaamyloid-related mitochondrial fission and neuronal injury. Science 324: 102-105, 2009.

94. Choi BY, Kim IY, Kim JH, Lee BE, Lee SH, Kho AR, Jung HJ, Sohn M, Song HK, and Suh SW. Decreased 
cysteine uptake by EAAC1 gene deletion exacerbates neuronal oxidative stress and neuronal death after traumatic brain injury. Amino Acids 48: 1619-1629, 2016.

95. Choi DH and Lee J. A Mini-review of the NADPH oxidases in vascular dementia: correlation with NOXs and risk factors for VaD. Int J Mol Sci 18: 2500, 2017.

96. Choi WS, Kruse SE, Palmiter RD, and Xia Z. Mitochondrial complex I inhibition is not required for dopaminergic neuron death induced by rotenone, $\mathrm{MPP}^{+}$, or paraquat. Proc Natl Acad Sci U S A 105: 15136-15141, 2008.

97. Chouchani ET, Pell VR, James AM, Work LM, SaebParsy K, Frezza C, Krieg T, and Murphy MP. A unifying mechanism for mitochondrial superoxide production during ischemia-reperfusion injury. Cell Metab 23: 254-263, 2016.

98. Chow J, Rahman J, Achermann JC, Dattani MT, and Rahman S. Mitochondrial disease and endocrine dysfunction. Nat Rev Endocrinol 13: 92-104, 2017.

99. Chung KK, Thomas B, Li X, Pletnikova O, Troncoso JC, Marsh L, Dawson VL, and Dawson TM. S-nitrosylation of parkin regulates ubiquitination and compromises parkin's protective function. Science 304: 1328-1331, 2004.

100. Clayton PT. Inherited disorders of transition metal metabolism: an update. J Inherit Metab Dis 40: 519-529, 2017.

101. Cleary JD and Ranum LP. New developments in RAN translation: insights from multiple diseases. Curr Opin Genet Dev 44: 125-134, 2017.

102. Cocco T, Sgobbo P, Clemente M, Lopriore B, Grattagliano I, Di Paola M, and Villani G. Tissue-specific changes of mitochondrial functions in aged rats: effect of a long-term dietary treatment with $\mathrm{N}$-acetylcysteine. Free Radic Biol Med 38: 796-805, 2005.

103. Coleman M and Blass JP. Autism and lactic acidosis. $J$ Autism Dev Disord 15: 1-8, 1985.

104. Commoner B, Townsend J, and Pake GE. Free radicals in biological materials. Nature 174: 689-691, 1954.

105. Conrad M, Schneider M, Seiler A, and Bornkamm GW. Physiological role of phospholipid hydroperoxide glutathione peroxidase in mammals. Biol Chem 388: 10191025, 2007.

106. Conway KA, Harper JD, and Lansbury PT. Accelerated in vitro fibril formation by a mutant alpha-synuclein linked to early-onset Parkinson disease. Nat Med 4: 1318-1320, 1998.

107. Corral-Debrinski M, Horton T, Lott MT, Shoffner JM, McKee AC, Beal MF, Graham BH, and Wallace DC. Marked changes in mitochondrial DNA deletion levels in Alzheimer brains. Genomics 23: 471-476, 1994.

108. Cossee M, Puccio H, Gansmuller A, Koutnikova H, Dierich A, LeMeur M, Fischbeck K, Dolle P, and Koenig M. Inactivation of the Friedreich ataxia mouse gene leads to early embryonic lethality without iron accumulation. Hum Mol Genet 9: 1219-1226, 2000.

109. Covarrubias-Pinto A, Acuna AI, Beltran FA, Torres-Diaz L, and Castro MA. Old things new view: ascorbic acid protects the brain in neurodegenerative disorders. Int $J$ Mol Sci 16: 28194-28217, 2015.

110. Covarrubias-Pinto A, Moll P, Solis-Maldonado M, Acuna AI, Riveros A, Miro MP, Papic E, Beltran FA, Cepeda C, Concha II, Brauchi S, and Castro MA. Beyond the redox imbalance: oxidative stress contributes to an impaired GLUT3 modulation in Huntington's disease. Free Radic Biol Med 89: 1085-1096, 2015.
111. Cozzolino M, Ferri A, and Carri MT. Amyotrophic lateral sclerosis: from current developments in the laboratory to clinical implications. Antioxid Redox Signal 10: 405-443, 2008.

112. Crielaard BJ, Lammers T, and Rivella S. Targeting iron metabolism in drug discovery and delivery. Nat Rev Drug Discov 16: 400-423, 2017.

113. Cui L, Jeong H, Borovecki F, Parkhurst CN, Tanese N, and Krainc D. Transcriptional repression of PGC-1alpha by mutant huntingtin leads to mitochondrial dysfunction and neurodegeneration. Cell 127: 59-69, 2006.

114. Cullinan SB, Gordan JD, Jin J, Harper JW, and Diehl JA. The Keap1-BTB protein is an adaptor that bridges Nrf2 to a Cul3-based E3 ligase: oxidative stress sensing by a Cul3-Keap1 ligase. Mol Cell Biol 24: 8477-8486, 2004.

115. Culotta VC, Klomp LW, Strain J, Casareno RL, Krems B, and Gitlin JD. The copper chaperone for superoxide dismutase. J Biol Chem 272: 23469-23472, 1997.

116. D'Souza AD, Parish IA, Krause DS, Kaech SM, and Shadel GS. Reducing mitochondrial ROS improves disease-related pathology in a mouse model of Ataxiatelangiectasia. Mol Ther 21: 42-48, 2013.

117. Dachsel JC and Farrer MJ. LRRK2 and Parkinson disease. Arch Neurol 67: 542-547, 2010.

118. Damiano M, Diguet E, Malgorn C, D’Aurelio M, Galvan L, Petit F, Benhaim L, Guillermier M, Houitte D, Dufour N, Hantraye P, Canals JM, Alberch J, Delzescaux T, Deglon N, Beal MF, and Brouillet E. A role of mitochondrial complex II defects in genetic models of Huntington's disease expressing N-terminal fragments of mutant huntingtin. Hum Mol Genet 22: 3869-3882, 2013.

119. Dardalhon M, Kumar C, Iraqui I, Vernis L, Kienda G, Banach-Latapy A, He T, Chanet R, Faye G, Outten CE, and Huang ME. Redox-sensitive YFP sensors monitor dynamic nuclear and cytosolic glutathione redox changes. Free Radic Biol Med 52: 2254-2265, 2012.

120. Davoli A, Greco V, Spalloni A, Guatteo E, Neri C, Rizzo GR, Cordella A, Romigi A, Cortese C, Bernardini S, Sarchielli P, Cardaioli G, Calabresi P, Mercuri NB, Urbani $\mathrm{A}$, and Longone P. Evidence of hydrogen sulfide involvement in amyotrophic lateral sclerosis. Ann Neurol 77: 697-709, 2015.

121. de Beus MD, Chung J, and Colon W. Modification of cysteine 111 in $\mathrm{Cu} / \mathrm{Zn}$ superoxide dismutase results in altered spectroscopic and biophysical properties. Protein Sci 13: 1347-1355, 2004.

122. De Grey AD. HO2*: the forgotten radical. DNA Cell Biol 21: 251-257, 2002.

123. de Haan JB, Bladier C, Griffiths P, Kelner M, O'Shea RD, Cheung NS, Bronson RT, Silvestro MJ, Wild S, Zheng SS, Beart PM, Hertzog PJ, and Kola I. Mice with a homozygous null mutation for the most abundant glutathione peroxidase, Gpx1, show increased susceptibility to the oxidative stress-inducing agents paraquat and hydrogen peroxide. J Biol Chem 273: 22528-22536, 1998.

124. Deas E, Cremades N, Angelova PR, Ludtmann MH, Yao Z, Chen S, Horrocks MH, Banushi B, Little D, Devine MJ, Gissen P, Klenerman D, Dobson CM, Wood NW, Gandhi $\mathrm{S}$, and Abramov AY. Alpha-synuclein oligomers interact with metal ions to induce oxidative stress and neuronal death in Parkinson's disease. Antioxid Redox Signal 24: 376-391, 2016. 
125. DeJesus-Hernandez M, Mackenzie IR, Boeve BF, Boxer AL, Baker M, Rutherford NJ, Nicholson AM, Finch NA, Flynn H, Adamson J, Kouri N, Wojtas A, Sengdy P, Hsiung GY, Karydas A, Seeley WW, Josephs KA, Coppola G, Geschwind DH, Wszolek ZK, Feldman H, Knopman DS, Petersen RC, Miller BL, Dickson DW, Boylan KB, Graff-Radford NR, and Rademakers R. Expanded GGGGCC hexanucleotide repeat in noncoding region of C9ORF72 causes chromosome 9p-linked FTD and ALS. Neuron 72: 245-256, 2011.

126. Delhey LM, Nur Kilinc E, Yin L, Slattery JC, Tippett ML, Rose S, Bennuri SC, Kahler SG, Damle S, Legido A, Goldenthal MJ, and Frye RE. The effect of mitochondrial supplements on mitochondrial activity in children with autism spectrum disorder. J Clin Med 6: pii: E18, 2017.

127. Deng H, Wang P, and Jankovic J. The genetics of Parkinson disease. Ageing Res Rev 42: 72-85, 2018.

128. Devasagayam TP, Sundquist AR, Di Mascio P, Kaiser S, and Sies H. Activity of thiols as singlet molecular oxygen quenchers. J Photochem Photobiol B 9: 105-116, 1991.

129. Devos D, Moreau C, Devedjian JC, Kluza J, Petrault M, Laloux C, Jonneaux A, Ryckewaert G, Garcon G, Rouaix N, Duhamel A, Jissendi P, Dujardin K, Auger F, Ravasi L, Hopes L, Grolez G, Firdaus W, Sablonniere B, StrubiVuillaume I, Zahr N, Destee A, Corvol JC, Poltl D, Leist M, Rose C, Defebvre L, Marchetti P, Cabantchik ZI, and Bordet R. Targeting chelatable iron as a therapeutic modality in Parkinson's disease. Antioxid Redox Signal 21: 195-210, 2014.

130. Dixon BM, Heath SH, Kim R, Suh JH, and Hagen TM. Assessment of endoplasmic reticulum glutathione redox status is confounded by extensive ex vivo oxidation. Antioxid Redox Signal 10: 963-972, 2008.

131. Dixon SJ, Lemberg KM, Lamprecht MR, Skouta R, Zaitsev EM, Gleason CE, Patel DN, Bauer AJ, Cantley AM, Yang WS, Morrison B, 3rd, and Stockwell BR. Ferroptosis: an iron-dependent form of nonapoptotic cell death. Cell 149: 1060-1072, 2012.

132. Do Van B, Gouel F, Jonneaux A, Timmerman K, Gele P, Petrault M, Bastide M, Laloux C, Moreau C, Bordet R, Devos D, and Devedjian JC. Ferroptosis, a newly characterized form of cell death in Parkinson's disease that is regulated by PKC. Neurobiol Dis 94: 169-178, 2016.

133. Dolske MC, Spollen J, McKay S, Lancashire E, and Tolbert L. A preliminary trial of ascorbic acid as supplemental therapy for autism. Prog NeuroPsychopharmacol Biol Psychiatry 17: 765-774, 1993.

134. Dryanovski DI, Guzman JN, Xie Z, Galteri DJ, VolpicelliDaley LA, Lee VM, Miller RJ, Schumacker PT, and Surmeier DJ. Calcium entry and alpha-synuclein inclusions elevate dendritic mitochondrial oxidant stress in dopaminergic neurons. J Neurosci 33: 10154-10164, 2013.

135. Duan $\mathbf{J}$ and Kasper DL. Oxidative depolymerization of polysaccharides by reactive oxygen/nitrogen species. Glycobiology 21: 401-409, 2011.

136. Dugan LL, Sensi SL, Canzoniero LM, Handran SD, Rothman SM, Lin TS, Goldberg MP, and Choi DW. Mitochondrial production of reactive oxygen species in cortical neurons following exposure to N-methyl-Daspartate. J Neurosci 15: 6377-6388, 1995.

137. Dunah AW, Jeong H, Griffin A, Kim YM, Standaert DG, Hersch SM, Mouradian MM, Young AB, Tanese N, and Krainc D. Sp1 and TAFII130 transcriptional activity dis- rupted in early Huntington's disease. Science 296: 22382243, 2002.

138. Earle KM. Studies on Parkinson's disease including X-ray fluorescent spectroscopy of formalin fixed brain tissue. $J$ Neuropathol Exp Neurol 27: 1-14, 1968.

139. Edge R, McGarvey DJ, and Truscott TG. The carotenoids as anti-oxidants-a review. J Photochem Photobiol B 41: 189-200, 1997.

140. Elchuri S, Oberley TD, Qi W, Eisenstein RS, Jackson Roberts L, Van Remmen H, Epstein CJ, and Huang TT. CuZnSOD deficiency leads to persistent and widespread oxidative damage and hepatocarcinogenesis later in life. Oncogene 24: 367-380, 2005.

141. Emir UE, Raatz S, McPherson S, Hodges JS, Torkelson C, Tawfik P, White T, and Terpstra M. Noninvasive quantification of ascorbate and glutathione concentration in the elderly human brain. NMR Biomed 24: 888-894, 2011.

142. Emmrich JV, Hornik TC, Neher JJ, and Brown GC. Rotenone induces neuronal death by microglial phagocytosis of neurons. FEBS J 280: 5030-5038, 2013.

143. Esworthy RS, Swiderek KM, Ho YS, and Chu FF. Seleniumdependent glutathione peroxidase-GI is a major glutathione peroxidase activity in the mucosal epithelium of rodent intestine. Biochim Biophys Acta 1381: 213-226, 1998.

144. Fang EF, Scheibye-Knudsen M, Chua KF, Mattson MP, Croteau DL, and Bohr VA. Nuclear DNA damage signalling to mitochondria in ageing. Nat Rev Mol Cell Biol 17: 308-321, 2016.

145. Ferguson G and Bridge W. Glutamate cysteine ligase and the age-related decline in cellular glutathione: the therapeutic potential of gamma-glutamylcysteine. Arch Biochem Biophys 593: 12-23, 2016.

146. Feron F, Gepner B, Lacassagne E, Stephan D, Mesnage B, Blanchard MP, Boulanger N, Tardif C, Deveze A, Rousseau S, Suzuki K, Izpisua Belmonte JC, Khrestchatisky M, Nivet E, and Erard-Garcia M. Olfactory stem cells reveal MOCOS as a new player in autism spectrum disorders. Mol Psychiatry 21: 1215-1224, 2016.

147. Ferri A, Cozzolino M, Crosio C, Nencini M, Casciati A, Gralla EB, Rotilio G, Valentine JS, and Carri MT. Familial ALS-superoxide dismutases associate with mitochondria and shift their redox potentials. Proc Natl Acad Sci U S A 103: 13860-13865, 2006.

148. Fetherolf MM, Boyd SD, Taylor AB, Kim HJ, Wohlschlegel JA, Blackburn NJ, Hart PJ, Winge DR, and Winkler DD. Copper-zinc superoxide dismutase is activated through a sulfenic acid intermediate at a copper ion entry site. J Biol Chem 292: 12025-12040, 2017.

149. Filomeni G, De Zio D, and Cecconi F. Oxidative stress and autophagy: the clash between damage and metabolic needs. Cell Death Differ 22: 377-388, 2015.

150. Finkel T. From sulfenylation to sulfhydration: what a thiolate needs to tolerate. Sci Signal 5: pe10, 2012.

151. Fisher AB. Peroxiredoxin 6: a bifunctional enzyme with glutathione peroxidase and phospholipase $\mathrm{A}_{2}$ activities. Antioxid Redox Signal 15: 831-844, 2011.

152. Fleming AM and Burrows CJ. Formation and processing of DNA damage substrates for the hNEIL enzymes. Free Radic Biol Med 107: 35-52, 2017.

153. Flohe L. The fairytale of the GSSG/GSH redox potential. Biochim Biophys Acta 1830: 3139-3142, 2013.

154. Flohe L, Toppo S, Cozza G, and Ursini F. A comparison of thiol peroxidase mechanisms. Antioxid Redox Signal 15: 763-780, 2011. 
155. Florian S, Krehl S, Loewinger M, Kipp A, Banning A, Esworthy S, Chu FF, and Brigelius-Flohe R. Loss of GPx2 increases apoptosis, mitosis, and GPx1 expression in the intestine of mice. Free Radic Biol Med 49: 1694-1702, 2010.

156. Florian S, Wingler K, Schmehl K, Jacobasch G, Kreuzer OJ, Meyerhof W, and Brigelius-Flohe R. Cellular and subcellular localization of gastrointestinal glutathione peroxidase in normal and malignant human intestinal tissue. Free Radic Res 35: 655-663, 2001.

157. Flors $\mathrm{C}$ and Nonell S. Light and singlet oxygen in plant defense against pathogens: phototoxic phenalenone phytoalexins. Acc Chem Res 39: 293-300, 2006.

158. Floyd RA and Carney JM. Free radical damage to protein and DNA: mechanisms involved and relevant observations on brain undergoing oxidative stress. Ann Neurol 32(Suppl): S22-S27, 1992.

159. Foury F and Cazzalini O. Deletion of the yeast homologue of the human gene associated with Friedreich's ataxia elicits iron accumulation in mitochondria. FEBS Lett 411 : 373-377, 1997.

160. Fox JH, Kama JA, Lieberman G, Chopra R, Dorsey K, Chopra V, Volitakis I, Cherny RA, Bush AI, and Hersch $\mathrm{S}$. Mechanisms of copper ion mediated Huntington's disease progression. PLoS One 2: e334, 2007.

161. Frederick NM, Bertho J, Patel KK, Petr GT, Bakradze E, Smith SB, and Rosenberg PA. Dysregulation of system $\mathrm{xc}(-)$ expression induced by mutant huntingtin in a striatal neuronal cell line and in R6/2 mice. Neurochem Int 76: 59-69, 2014.

162. Freedman RB, Desmond JL, Byrne LJ, Heal JW, Howard MJ, Sanghera N, Walker KL, Wallis AK, Wells SA, Williamson RA, and Romer RA. "Something in the way she moves": the functional significance of flexibility in the multiple roles of protein disulfide isomerase (PDI). Biochim Biophys Acta 1865: 1383-1394, 2017.

163. Frustaci A, Neri M, Cesario A, Adams JB, Domenici E, Dalla Bernardina B, and Bonassi S. Oxidative stressrelated biomarkers in autism: systematic review and metaanalyses. Free Radic Biol Med 52: 2128-2141, 2012.

164. Frye RE and Rossignol DA. Treatments for biomedical abnormalities associated with autism spectrum disorder. Front Pediatr 2: 66, 2014.

165. Fukai T and Ushio-Fukai M. Superoxide dismutases: role in redox signaling, vascular function, and diseases. Antioxid Redox Signal 15: 1583-1606, 2011.

166. Gadoth N and Goebel HH. Oxidative Stress and Free Radical Damage in Neurology. New York: Humana Press: Springer, 2011, viii, 323 p.

167. Gamberino WC and Brennan WA, Jr. Glucose transporter isoform expression in Huntington's disease brain. $\mathrm{J} \mathrm{Neu-}$ rochem 63: 1392-1397, 1994.

168. Gao HM, Zhang F, Zhou H, Kam W, Wilson B, and Hong JS. Neuroinflammation and alpha-synuclein dysfunction potentiate each other, driving chronic progression of neurodegeneration in a mouse model of Parkinson's disease. Environ Health Perspect 119: 807-814, 2011.

169. Gao XH, Krokowski D, Guan BJ, Bederman I, Majumder M, Parisien M, Diatchenko L, Kabil O, Willard B, Banerjee R, Wang B, Bebek G, Evans CR, Fox PL, Gerson SL, Hoppel CL, Liu M, Arvan P, and Hatzoglou M. Quantitative $\mathrm{H}_{2} \mathrm{~S}$-mediated protein sulfhydration reveals metabolic reprogramming during the integrated stress response. Elife 4: e10067, 2015.
170. Gatt AP, Duncan OF, Attems J, Francis PT, Ballard CG, and Bateman JM. Dementia in Parkinson's disease is associated with enhanced mitochondrial complex I deficiency. Mov Disord 31: 352-359, 2016.

171. Gautam AH and Zeevalk GD. Characterization of reduced and oxidized dopamine and 3,4-dihydrophenylacetic acid, on brain mitochondrial electron transport chain activities. Biochim Biophys Acta 1807: 819-828, 2011.

172. Ghanizadeh A and Derakhshan N. N-acetylcysteine for treatment of autism, a case report. J Res Med Sci 17: 985987, 2012.

173. Ghezzi P. Regulation of protein function by glutathionylation. Free Radic Res 39: 573-580, 2005.

174. Ghezzi P, Bonetto V, and Fratelli M. Thiol-disulfide balance: from the concept of oxidative stress to that of redox regulation. Antioxid Redox Signal 7: 964-972, 2005.

175. Ghezzi $\mathrm{P}$ and Chan P. Redox proteomics applied to the thiol secretome. Antioxid Redox Signal 26: 299-312, 2017.

176. Gijselinck I, Van Langenhove T, van der Zee J, Sleegers K, Philtjens S, Kleinberger G, Janssens J, Bettens K, Van Cauwenberghe C, Pereson S, Engelborghs S, Sieben A, De Jonghe P, Vandenberghe R, Santens P, De Bleecker J, Maes G, Baumer V, Dillen L, Joris G, Cuijt I, Corsmit E, Elinck E, Van Dongen J, Vermeulen S, Van den Broeck M, Vaerenberg C, Mattheijssens M, Peeters K, Robberecht W, Cras P, Martin JJ, De Deyn PP, Cruts M, and Van Broeckhoven C. A C9orf72 promoter repeat expansion in a Flanders-Belgian cohort with disorders of the frontotemporal lobar degeneration-amyotrophic lateral sclerosis spectrum: a gene identification study. Lancet Neurol 11: 54-65, 2012.

177. Giles GI, Nasim MJ, Ali W, and Jacob C. The reactive sulfur species concept: 15 years on. Antioxidants (Basel) 6: E38, 2017.

178. Giles GI, Tasker KM, and Jacob C. Hypothesis: the role of reactive sulfur species in oxidative stress. Free Radic Biol Med 31: 1279-1283, 2001.

179. Glass CK, Saijo K, Winner B, Marchetto MC, and Gage FH. Mechanisms underlying inflammation in neurodegeneration. Cell 140: 918-934, 2010.

180. Glorieux C and Calderon PB. Catalase, a remarkable enzyme: targeting the oldest antioxidant enzyme to find a new cancer treatment approach. Biol Chem 398: 10951108, 2017.

181. Goedert M, Spillantini MG, Del Tredici K, and Braak H. 100 Years of Lewy pathology. Nat Rev Neurol 9: 13-24, 2013.

182. Gonatas NK, Stieber A, and Gonatas JO. Fragmentation of the Golgi apparatus in neurodegenerative diseases and cell death. J Neurol Sci 246: 21-30, 2006.

183. Gordon LB, Rothman FG, Lopez-Otin C, and Misteli T. Progeria: a paradigm for translational medicine. Cell 156: 400-407, 2014.

184. Graham DG, Tiffany SM, Bell WR, Jr., and Gutknecht WF. Autoxidation versus covalent binding of quinones as the mechanism of toxicity of dopamine, 6-hydroxydopamine, and related compounds toward C1300 neuroblastoma cells in vitro. Mol Pharmacol 14: 644-653, 1978.

185. Grootveld M, Henderson EB, Farrell A, Blake DR, Parkes HG, and Haycock P. Oxidative damage to hyaluronate and glucose in synovial fluid during exercise of the inflamed rheumatoid joint. Detection of abnormal low-molecularmass metabolites by proton-n.m.r. spectroscopy. Biochem J 273(Pt 2): 459-467, 1991. 
186. Gruber CW, Cemazar M, Heras B, Martin JL, and Craik DJ. Protein disulfide isomerase: the structure of oxidative folding. Trends Biochem Sci 31: 455-464, 2006.

187. Gruhlke MC and Slusarenko AJ. The biology of reactive sulfur species (RSS). Plant Physiol Biochem 59: 98-107, 2012.

188. Gu F, Chauhan V, and Chauhan A. Impaired synthesis and antioxidant defense of glutathione in the cerebellum of autistic subjects: alterations in the activities and protein expression of glutathione-related enzymes. Free Radic Biol Med 65: 488-496, 2013.

189. Gu M, Gash MT, Mann VM, Javoy-Agid F, Cooper JM, and Schapira AH. Mitochondrial defect in Huntington's disease caudate nucleus. Ann Neurol 39: 385-389, 1996.

190. Gu X, Greiner ER, Mishra R, Kodali R, Osmand A, Finkbeiner S, Steffan JS, Thompson LM, Wetzel R, and Yang XW. Serines 13 and 16 are critical determinants of full-length human mutant huntingtin induced disease pathogenesis in HD mice. Neuron 64: 828-840, 2009.

191. Guglielmotto M, Monteleone D, Giliberto L, Fornaro M, Borghi R, Tamagno E, and Tabaton M. Amyloid- $\beta_{42}$ activates the expression of BACE1 through the JNK pathway. J Alzheimers Dis 27: 871-883, 2011.

192. Guo C, Sun L, Chen X, and Zhang D. Oxidative stress, mitochondrial damage and neurodegenerative diseases. Neural Regen Res 8: 2003-2014, 2013.

193. Guo X and Qi X. VCP cooperates with UBXD1 to degrade mitochondrial outer membrane protein MCL1 in model of Huntington's disease. Biochim Biophys Acta 1863: 552-559, 2017.

194. Guo X, Sun X, Hu D, Wang YJ, Fujioka H, Vyas R, Chakrapani S, Joshi AU, Luo Y, Mochly-Rosen D, and Qi $\mathrm{X}$. VCP recruitment to mitochondria causes mitophagy impairment and neurodegeneration in models of Huntington's disease. Nat Commun 7: 12646, 2016.

195. Guo Z, Kozlov S, Lavin MF, Person MD, and Paull TT. ATM activation by oxidative stress. Science 330: 517-521, 2010.

196. Gurney ME, Cutting FB, Zhai P, Doble A, Taylor CP, Andrus PK, and Hall ED. Benefit of vitamin E, riluzole, and gabapentin in a transgenic model of familial amyotrophic lateral sclerosis. Ann Neurol 39: 147-157, 1996.

197. Gurney ME, Pu H, Chiu AY, Dal Canto MC, Polchow CY, Alexander DD, Caliendo J, Hentati A, Kwon YW, and Deng HX, Chen W, Zhai P, Sufit RL, and Siddique T. Motor neuron degeneration in mice that express a human $\mathrm{Cu}, \mathrm{Zn}$ superoxide dismutase mutation. Science 264: 1772-1775, 1994.

198. Guzman JN, Sanchez-Padilla J, Wokosin D, Kondapalli J, Ilijic E, Schumacker PT, and Surmeier DJ. Oxidant stress evoked by pacemaking in dopaminergic neurons is attenuated by DJ-1. Nature 468: 696-700, 2010.

199. Gvozdjakova A, Kucharska J, Ostatnikova D, Babinska K, Nakladal D, and Crane FL. Ubiquinol improves symptoms in children with autism. Oxid Med Cell Longev 2014: 798957, 2014.

200. Haeusler AR, Donnelly CJ, Periz G, Simko EA, Shaw PG, Kim MS, Maragakis NJ, Troncoso JC, Pandey A, Sattler $\mathrm{R}$, Rothstein JD, and Wang J. C9orf72 nucleotide repeat structures initiate molecular cascades of disease. Nature 507: 195-200, 2014.

201. Haeusler AR, Donnelly CJ, and Rothstein JD. The expanding biology of the C9orf72 nucleotide repeat expansion in neurodegenerative disease. Nat Rev Neurosci 17: 383-395, 2016.
202. Halliwell B. Reactive oxygen species and the central nervous system. J Neurochem 59: 1609-1623, 1992.

203. Halliwell B. The antioxidant paradox. Lancet 355: 11791180, 2000.

204. Halliwell B. Role of free radicals in the neurodegenerative diseases: therapeutic implications for antioxidant treatment. Drugs Aging 18: 685-716, 2001.

205. Halliwell B. Free radicals and antioxidants-quo vadis? Trends Pharmacol Sci 32: 125-130, 2011.

206. Halliwell B. The antioxidant paradox: less paradoxical now? Br J Clin Pharmacol 75: 637-644, 2013.

207. Halliwell B, Hu ML, Louie S, Duvall TR, Tarkington BK, Motchnik P, and Cross CE. Interaction of nitrogen dioxide with human plasma. Antioxidant depletion and oxidative damage. FEBS Lett 313: 62-66, 1992.

208. Hanzen S, Vielfort K, Yang J, Roger F, Andersson V, Zamarbide-Fores S, Andersson R, Malm L, Palais G, Biteau B, Liu B, Toledano MB, Molin M, and Nystrom T. Lifespan control by redox-dependent recruitment of chaperones to misfolded proteins. Cell 166: 140-151, 2016.

209. Hara MR, Agrawal N, Kim SF, Cascio MB, Fujimuro M, Ozeki Y, Takahashi M, Cheah JH, Tankou SK, Hester LD, Ferris CD, Hayward SD, Snyder SH, and Sawa A. Snitrosylated GAPDH initiates apoptotic cell death by nuclear translocation following Siah1 binding. Nat Cell Biol 7: 665-674, 2005.

210. Hardan AY, Fung LK, Libove RA, Obukhanych TV, Nair S, Herzenberg LA, Frazier TW, and Tirouvanziam R. A randomized controlled pilot trial of oral $\mathrm{N}$-acetylcysteine in children with autism. Biol Psychiatry 71: 956-961, 2012.

211. Harding AE. Classification of the hereditary ataxias and paraplegias. Lancet 1: 1151-1155, 1983.

212. Hastings TG, Lewis DA, and Zigmond MJ. Role of oxidation in the neurotoxic effects of intrastriatal dopamine injections. Proc Natl Acad Sci U S A 93: 1956-1961, 1996.

213. Hayes JD and Dinkova-Kostova AT. The Nrf2 regulatory network provides an interface between redox and intermediary metabolism. Trends Biochem Sci 39: 199-218, 2014.

214. Heikkila RE, Manzino L, Cabbat FS, and Duvoisin RC. Protection against the dopaminergic neurotoxicity of 1methyl-4-phenyl-1,2,5,6-tetrahydropyridine by monoamine oxidase inhibitors. Nature 311: 467-469, 1984.

215. Helferich AM, Ruf WP, Grozdanov V, Freischmidt A, Feiler MS, Zondler L, Ludolph AC, McLean PJ, Weishaupt JH, and Danzer KM. Alpha-synuclein interacts with SOD1 and promotes its oligomerization. Mol Neurodegener 10: 66, 2015.

216. Herrera AJ, Castano A, Venero JL, Cano J, and Machado A. The single intranigral injection of LPS as a new model for studying the selective effects of inflammatory reactions on dopaminergic system. Neurobiol Dis 7: 429-447, 2000.

217. Hersch SM, Gevorkian S, Marder K, Moskowitz C, Feigin A, Cox M, Como P, Zimmerman C, Lin M, Zhang L, Ulug AM, Beal MF, Matson W, Bogdanov M, Ebbel E, Zaleta A, Kaneko Y, Jenkins B, Hevelone N, Zhang H, Yu H, Schoenfeld D, Ferrante R, and Rosas HD. Creatine in Huntington disease is safe, tolerable, bioavailable in brain and reduces serum $8 \mathrm{OH} 2^{\prime} \mathrm{dG}$. Neurology 66: 250-252, 2006.

218. Hine C, Harputlugil E, Zhang Y, Ruckenstuhl C, Lee BC, Brace L, Longchamp A, Trevino-Villarreal JH, Mejia P, Ozaki CK, Wang R, Gladyshev VN, Madeo F, Mair WB, and Mitchell JR. Endogenous hydrogen sulfide production 
is essential for dietary restriction benefits. Cell 160: 132 $144,2015$.

219. Hirano A, Nakano I, Kurland LT, Mulder DW, Holley PW, and Saccomanno G. Fine structural study of neurofibrillary changes in a family with amyotrophic lateral sclerosis. J Neuropathol Exp Neurol 43: 471-480, 1984.

220. Hirsch EC, Hunot S, Damier P, and Faucheux B. Glial cells and inflammation in Parkinson's disease: a role in neurodegeneration? Ann Neurol 44: S115-S120, 1998.

221. Ho YS, Gargano M, Cao J, Bronson RT, Heimler I, and Hutz RJ. Reduced fertility in female mice lacking copperzinc superoxide dismutase. J Biol Chem 273: 7765-7769, 1998.

222. Ho YS, Xiong Y, Ma W, Spector A, and Ho DS. Mice lacking catalase develop normally but show differential sensitivity to oxidant tissue injury. J Biol Chem 279: 32804-32812, 2004.

223. Hodgson N, Trivedi M, Muratore C, Li S, and Deth R. Soluble oligomers of amyloid-beta cause changes in redox state, DNA methylation, and gene transcription by inhibiting EAAT3 mediated cysteine uptake. J Alzheimers Dis 36: 197-209, 2013.

224. Holmstrom KM, Kostov RV, and Dinkova-Kostova AT. The multifaceted role of Nrf2 in mitochondrial function. Curr Opin Toxicol 1: 80-91, 2016.

225. Hopes L, Grolez G, Moreau C, Lopes R, Ryckewaert G, Carriere N, Auger F, Laloux C, Petrault M, Devedjian JC, Bordet R, Defebvre L, Jissendi P, Delmaire C, and Devos D. Magnetic resonance imaging features of the nigrostriatal system: biomarkers of Parkinson's disease stages? PLoS One 11: e0147947, 2016.

226. $\mathrm{Hu} \mathrm{BR}$, Martone ME, Jones YZ, and Liu CL. Protein aggregation after transient cerebral ischemia. J Neurosci 20: 3191-3199, 2000.

227. Hu LF, Lu M, Tiong CX, Dawe GS, Hu G, and Bian JS. Neuroprotective effects of hydrogen sulfide on Parkinson's disease rat models. Aging Cell 9: 135-146, 2010.

228. Huang TT, Carlson EJ, Gillespie AM, Shi Y, and Epstein CJ. Ubiquitous overexpression of $\mathrm{CuZn}$ superoxide dismutase does not extend life span in mice. $J$ Gerontol $A$ Biol Sci Med Sci 55: B5-B9, 2000.

229. Huang X, Atwood CS, Hartshorn MA, Multhaup G, Goldstein LE, Scarpa RC, Cuajungco MP, Gray DN, Lim J, Moir RD, Tanzi RE, and Bush AI. The A beta peptide of Alzheimer's disease directly produces hydrogen peroxide through metal ion reduction. Biochemistry 38: 7609-7616, 1999.

230. Huang X, Cuajungco MP, Atwood CS, Hartshorn MA, Tyndall JD, Hanson GR, Stokes KC, Leopold M, Multhaup G, Goldstein LE, Scarpa RC, Saunders AJ, Lim J, Moir RD, Glabe C, Bowden EF, Masters CL, Fairlie DP, Tanzi RE, and Bush AI. Cu(II) potentiation of Alzheimer abeta neurotoxicity. Correlation with cell-free hydrogen peroxide production and metal reduction. $J$ Biol Chem 274: 37111-37116, 1999.

231. Hung RJ, Yazdani U, Yoon J, Wu H, Yang T, Gupta N, Huang Z, van Berkel WJ, and Terman JR. Mical links semaphorins to F-actin disassembly. Nature 463: 823-827, 2010.

232. Huntington Study Group. Tetrabenazine as antichorea therapy in Huntington disease: a randomized controlled trial. Neurology 66: 366-372, 2006.

233. Hurley MJ, Brandon B, Gentleman SM, and Dexter DT. Parkinson's disease is associated with altered expression of $\mathrm{CaV} 1$ channels and calcium-binding proteins. Brain 136: 2077-2097, 2013.
234. Hwang C, Sinskey AJ, and Lodish HF. Oxidized redox state of glutathione in the endoplasmic reticulum. Science 257: 1496-1502, 1992.

235. Ichida K, Matsumura T, Sakuma R, Hosoya T, and Nishino T. Mutation of human molybdenum cofactor sulfurase gene is responsible for classical xanthinuria type II. Biochem Biophys Res Commun 282: 1194-1200, 2001.

236. Ignarro LJ. Biosynthesis and metabolism of endotheliumderived nitric oxide. Апnи Rev Pharmacol Toxicol 30: 535-560, 1990.

237. Ilijic E, Guzman JN, and Surmeier DJ. The L-type channel antagonist isradipine is neuroprotective in a mouse model of Parkinson's disease. Neurobiol Dis 43: 364-371, 2011.

238. Imamura Y, Noda S, Hashizume K, Shinoda K, Yamaguchi M, Uchiyama S, Shimizu T, Mizushima Y, Shirasawa T, and Tsubota K. Drusen, choroidal neovascularization, and retinal pigment epithelium dysfunction in SOD1-deficient mice: a model of age-related macular degeneration. Proc Natl Acad Sci U S A 103: 11282-11287, 2006.

239. Imlay JA, Chin SM, and Linn S. Toxic DNA damage by hydrogen peroxide through the Fenton reaction in vivo and in vitro. Science 240: 640-642, 1988.

240. Itoh K, Wakabayashi N, Katoh Y, Ishii T, Igarashi K, Engel JD, and Yamamoto M. Keap1 represses nuclear activation of antioxidant responsive elements by $\mathrm{Nrf} 2$ through binding to the amino-terminal Neh2 domain. Genes Dev 13: 76-86, 1999.

241. Jackson MR, Melideo SL, and Jorns MS. Human sulfide: quinone oxidoreductase catalyzes the first step in hydrogen sulfide metabolism and produces a sulfane sulfur metabolite. Biochemistry 51: 6804-6815, 2012.

242. James SJ, Cutler P, Melnyk S, Jernigan S, Janak L, Gaylor DW, and Neubrander JA. Metabolic biomarkers of increased oxidative stress and impaired methylation capacity in children with autism. Am J Clin Nutr 80: 1611-1617, 2004.

243. James SJ, Melnyk S, Fuchs G, Reid T, Jernigan S, Pavliv O, Hubanks A, and Gaylor DW. Efficacy of methylcobalamin and folinic acid treatment on glutathione redox status in children with autism. Am J Clin Nutr 89: 425430, 2009.

244. James SJ, Melnyk S, Jernigan S, Hubanks A, Rose S, and Gaylor DW. Abnormal transmethylation/transsulfuration metabolism and DNA hypomethylation among parents of children with autism. J Autism Dev Disord 38: 19661975, 2008.

245. Javitch JA, D'Amato RJ, Strittmatter SM, and Snyder SH. Parkinsonism-inducing neurotoxin, N-methyl-4-phenyl1,2,3,6-tetrahydropyridine: uptake of the metabolite $\mathrm{N}$ methyl-4-phenylpyridine by dopamine neurons explains selective toxicity. Proc Natl Acad Sci U S A 82: 2173 2177, 1985.

246. Jeng W, Loniewska MM, and Wells PG. Brain glucose-6phosphate dehydrogenase protects against endogenous oxidative DNA damage and neurodegeneration in aged mice. ACS Chem Neurosci 4: 1123-1132, 2013.

247. Jenkins BG, Koroshetz WJ, Beal MF, and Rosen BR. Evidence for impairment of energy metabolism in vivo in Huntington's disease using localized 1H NMR spectroscopy. Neurology 43: 2689-2695, 1993.

248. Jin RC, Mahoney CE, Coleman Anderson L, Ottaviano F, Croce K, Leopold JA, Zhang YY, Tang SS, Handy DE, and Loscalzo J. Glutathione peroxidase-3 deficiency promotes platelet-dependent thrombosis in vivo. Circulation 123: 1963-1973, 2011. 
249. Jin YN, Yu YV, Gundemir S, Jo C, Cui M, Tieu K, and Johnson GV. Impaired mitochondrial dynamics and Nrf2 signaling contribute to compromised responses to oxidative stress in striatal cells expressing full-length mutant huntingtin. PLoS One 8: e57932, 2013.

250. Jiralerspong S, Ge B, Hudson TJ, and Pandolfo $\mathrm{M}$. Manganese superoxide dismutase induction by iron is impaired in Friedreich ataxia cells. FEBS Lett 509: 101105, 2001.

251. Johri A, Chandra A, and Beal MF. PGC-1alpha, mitochondrial dysfunction, and Huntington's disease. Free Radic Biol Med 62: 37-46, 2013.

252. Jones DP. Redefining oxidative stress. Antioxid Redox Signal 8: 1865-1879, 2006.

253. Jones DP, Carlson JL, Mody VC, Cai J, Lynn MJ, and Sternberg P. Redox state of glutathione in human plasma. Free Radic Biol Med 28: 625-635, 2000.

254. Jones DP, Mody VC, Jr., Carlson JL, Lynn MJ, and Sternberg P, Jr. Redox analysis of human plasma allows separation of pro-oxidant events of aging from decline in antioxidant defenses. Free Radic Biol Med 33: 12901300, 2002.

255. Joshi G, Chi Y, Huang Z, and Wang Y. Abeta-induced Golgi fragmentation in Alzheimer's disease enhances Abeta production. Proc Natl Acad Sci U S A 9: E1230E1239, 2014.

256. Jozefczuk J, Kasprzycka W, Czarnecki R, Graczyk A, Jozefczuk P, Magda K, and Lampart U. Homocysteine as a diagnostic and etiopathogenic factor in children with autism spectrum disorder. J Med Food 20: 744-749, 2017.

257. Kabil H, Kabil O, Banerjee R, Harshman LG, and Pletcher SD. Increased transsulfuration mediates longevity and dietary restriction in Drosophila. Proc Natl Acad Sci U S A 108: 16831-16836, 2011.

258. Kamel F. Epidemiology. Paths from pesticides to Parkinson's. Science 341: 722-723, 2013.

259. Kawamata $\mathrm{H}$ and Manfredi G. Different regulation of wild-type and mutant $\mathrm{Cu}, \mathrm{Zn}$ superoxide dismutase localization in mammalian mitochondria. Hum Mol Genet 17: 3303-3317, 2008.

260. Kawamata H and Manfredi G. Import, maturation, and function of SOD1 and its copper chaperone CCS in the mitochondrial intermembrane space. Antioxid Redox Signal 13: 1375-1384, 2010.

261. Keeney PM, Xie J, Capaldi RA, and Bennett JP, Jr. Parkinson's disease brain mitochondrial complex I has oxidatively damaged subunits and is functionally impaired and misassembled. J Neurosci 26: 5256-5264, 2006.

262. Kehrer JP. The haber-Weiss reaction and mechanisms of toxicity. Toxicology 149: 43-50, 2000.

263. Kell DB. Towards a unifying, systems biology understanding of large-scale cellular death and destruction caused by poorly liganded iron: Parkinson's, Huntington's, Alzheimer's, prions, bactericides, chemical toxicology and others as examples. Arch Toxicol 84: 825-889, 2010.

264. Kemp M, Go YM, and Jones DP. Nonequilibrium thermodynamics of thiol/disulfide redox systems: a perspective on redox systems biology. Free Radic Biol Med 44: 921-937, 2008.

265. Kervern M, Angeli A, Nicole O, Leveille F, Parent B, Villette V, Buisson A, and Dutar P. Selective impairment of some forms of synaptic plasticity by oligomeric amyloid-beta peptide in the mouse hippocampus: impli- cation of extrasynaptic NMDA receptors. $J$ Alzheimers Dis 32: 183-196, 2012.

266. Kida K, Yamada M, Tokuda K, Marutani E, Kakinohana M, Kaneki M, and Ichinose F. Inhaled hydrogen sulfide prevents neurodegeneration and movement disorder in a mouse model of Parkinson's disease. Antioxid Redox Signal 15: 343-352, 2011.

267. Kim WG, Mohney RP, Wilson B, Jeohn GH, Liu B, and Hong JS. Regional difference in susceptibility to lipopolysaccharide-induced neurotoxicity in the rat brain: role of microglia. J Neurosci 20: 6309-6316, 2000.

268. Kimura H. Signaling molecules: hydrogen sulfide and polysulfide. Antioxid Redox Signal 22: 362-376, 2015.

269. Kirsch M, Korth HG, Sustmann R, and de Groot H. The pathobiochemistry of nitrogen dioxide. Biol Chem 383: 389-399, 2002.

270. Klepac N, Relja M, Klepac R, Hecimovic S, Babic T, and Trkulja V. Oxidative stress parameters in plasma of Huntington's disease patients, asymptomatic Huntington's disease gene carriers and healthy subjects: a crosssectional study. J Neurol 254: 1676-1683, 2007.

271. Klivenyi P, Andreassen OA, Ferrante RJ, Dedeoglu A, Mueller G, Lancelot E, Bogdanov M, Andersen JK, Jiang $\mathrm{D}$, and Beal MF. Mice deficient in cellular glutathione peroxidase show increased vulnerability to malonate, 3 nitropropionic acid, and 1-methyl-4-phenyl-1,2,5,6tetrahydropyridine. J Neurosci 20: 1-7, 2000.

272. Klockgether T and Paulson H. Milestones in ataxia. Mov Disord 26: 1134-1141, 2011.

273. Kobayashi A, Kang MI, Okawa H, Ohtsuji M, Zenke Y, Chiba T, Igarashi K, and Yamamoto M. Oxidative stress sensor Keap1 functions as an adaptor for Cul3-based E3 ligase to regulate proteasomal degradation of $\mathrm{Nrf2}$. Mol Cell Biol 24: 7130-7139, 2004.

274. Komatsu M, Kurokawa H, Waguri S, Taguchi K, Kobayashi A, Ichimura Y, Sou YS, Ueno I, Sakamoto A, Tong KI, Kim M, Nishito Y, Iemura S, Natsume T, Ueno $\mathrm{T}$, Kominami E, Motohashi $\mathrm{H}$, Tanaka K, and Yamamoto M. The selective autophagy substrate p62 activates the stress responsive transcription factor Nrf2 through inactivation of Keap1. Nat Cell Biol 12: 213-223, 2010.

275. Korcok J, Dixon SJ, Lo TC, and Wilson JX. Differential effects of glucose on dehydroascorbic acid transport and intracellular ascorbate accumulation in astrocytes and skeletal myocytes. Brain Res 993: 201-207, 2003.

276. Koroshetz WJ, Jenkins BG, Rosen BR, and Beal MF. Energy metabolism defects in Huntington's disease and effects of coenzyme Q10. Ann Neurol 41: 160-165, 1997.

277. Koutnikova H, Campuzano V, Foury F, Dolle P, Cazzalini $\mathrm{O}$, and Koenig M. Studies of human, mouse and yeast homologues indicate a mitochondrial function for frataxin. Nat Genet 16: 345-351, 1997.

278. Kovtun IV, Liu Y, Bjoras M, Klungland A, Wilson SH, and McMurray CT. OGG1 initiates age-dependent CAG trinucleotide expansion in somatic cells. Nature 447: 447452, 2007.

279. Krieger-Liszkay A and Trebst A. Tocopherol is the scavenger of singlet oxygen produced by the triplet states of chlorophyll in the PSII reaction centre. J Exp Bot 57: 1677-1684, 2006.

280. Kronenfeld G, Engelman R, Weisman-Shomer P, Atlas D, and Benhar M. Thioredoxin-mimetic peptides as catalysts of S-denitrosylation and anti-nitrosative stress agents. Free Radic Biol Med 79: 138-146, 2015. 
281. Kubben N, Zhang W, Wang L, Voss TC, Yang J, Qu J, Liu GH, and Misteli T. Repression of the antioxidant NRF2 pathway in premature aging. Cell 165: 1361-1374, 2016.

282. Kumar A and Ratan RR. Oxidative stress and Huntington's disease: the good, the bad, and the ugly. J Huntingtons Dis 5: 217-237, 2016.

283. Kumar R, Jangir DK, Verma G, Shekhar S, Hanpude P, Kumar S, Kumari R, Singh N, Sarovar BN, Ranjan Jana N, and Kanti Maiti T. S-nitrosylation of UCHL1 induces its structural instability and promotes alpha-synuclein aggregation. Sci Rep 7: 44558, 2017.

284. Kuwabara Y, Nishino T, Okamoto K, Matsumura T, Eger BT, Pai EF, and Nishino T. Unique amino acids cluster for switching from the dehydrogenase to oxidase form of xanthine oxidoreductase. Proc Natl Acad Sci U S A 100: 8170-8175, 2003.

285. Lai MC, Lombardo MV, and Baron-Cohen S. Autism. Lancet 383: 896-910, 2014.

286. Lamb AL, Torres AS, O'Halloran TV, and Rosenzweig AC. Heterodimeric structure of superoxide dismutase in complex with its metallochaperone. Nat Struct Biol 8: 751-755, 2001.

287. Lambeth JD and Neish AS. Nox enzymes and new thinking on reactive oxygen: a double-edged sword revisited. Annu Rev Pathol 9: 119-145, 2014.

288. Langston JW. The MPTP story. J Parkinsons Dis 7: S11S22, 2017.

289. Langston JW, Ballard P, Tetrud JW, and Irwin I. Chronic parkinsonism in humans due to a product of meperidineanalog synthesis. Science 219: 979-980, 1983.

290. Langston JW, Irwin I, Langston EB, and Forno LS. 1Methyl-4-phenylpyridinium ion $\left(\mathrm{MPP}^{+}\right)$: identification of a metabolite of MPTP, a toxin selective to the substantia nigra. Neurosci Lett 48: 87-92, 1984.

291. Lasalle JM. Autism genes keep turning up chromatin. $O A$ Autism 1: 14, 2013.

292. Latunde-Dada GO. Ferroptosis: role of lipid peroxidation, iron and ferritinophagy. Biochim Biophys Acta 1861: 1893-1900, 2017.

293. Lazarow PB and de Duve C. The synthesis and turnover of rat liver peroxisomes. V. Intracellular pathway of catalase synthesis. J Cell Biol 59: 507-524, 1973.

294. Leavitt BR and Hayden MR. Is tetrabenazine safe and effective for suppressing chorea in Huntington's disease? Nat Clin Pract Neurol 2: 536-537, 2006.

295. Lebovitz RM, Zhang H, Vogel H, Cartwright J, Jr., Dionne L, Lu N, Huang S, and Matzuk MM. Neurodegeneration, myocardial injury, and perinatal death in mitochondrial superoxide dismutase-deficient mice. Proc Natl Acad Sci U S A 93: 9782-9787, 1996.

296. Lee BC, Peterfi Z, Hoffmann FW, Moore RE, Kaya A, Avanesov A, Tarrago L, Zhou Y, Weerapana E, Fomenko DE, Hoffmann PR, and Gladyshev VN. MsrB1 and MICALs regulate actin assembly and macrophage function via reversible stereoselective methionine oxidation. $\mathrm{Mol}$ Cell 51: 397-404, 2013.

297. Lee J, Giordano S, and Zhang J. Autophagy, mitochondria and oxidative stress: cross-talk and redox signalling. Biochem J 441: 523-540, 2012.

298. Lei XG, Zhu JH, Cheng WH, Bao Y, Ho YS, Reddi AR, Holmgren A, and Arner ES. Paradoxical roles of antioxidant enzymes: basic mechanisms and health implications. Physiol Rev 96: 307-364, 2016.
299. Levine TP, Daniels RD, Gatta AT, Wong LH, and Hayes MJ. The product of C9orf72, a gene strongly implicated in neurodegeneration, is structurally related to DENN RabGEFs. Bioinformatics 29: 499-503, 2013.

300. Levonen AL, Hill BG, Kansanen E, Zhang J, and DarleyUsmar VM. Redox regulation of antioxidants, autophagy, and the response to stress: implications for electrophile therapeutics. Free Radic Biol Med 71: 196-207, 2014.

301. Lhermitte J, Kraus WM, and McAlpine D. Original papers: on the occurrence of abnormal deposits of iron in the brain in parkinsonism with special reference to its localisation. J Neurol Psychopathol 5: 195-208, 1924.

302. Li S, Jin M, Koeglsperger T, Shepardson NE, Shankar GM, and Selkoe DJ. Soluble Abeta oligomers inhibit longterm potentiation through a mechanism involving excessive activation of extrasynaptic NR2B-containing NMDA receptors. J Neurosci 31: 6627-6638, 2011.

303. Li X, Valencia A, Sapp E, Masso N, Alexander J, Reeves P, Kegel KB, Aronin N, and Difiglia M. Aberrant Rab11dependent trafficking of the neuronal glutamate transporter EAAC1 causes oxidative stress and cell death in Huntington's disease. J Neurosci 30: 4552-4561, 2010.

304. Libiad M, Yadav PK, Vitvitsky V, Martinov M, and Banerjee R. Organization of the human mitochondrial hydrogen sulfide oxidation pathway. J Biol Chem 289: 30901-30910, 2014.

305. Lin MT and Beal MF. Mitochondrial dysfunction and oxidative stress in neurodegenerative diseases. Nature 443: 787-795, 2006.

306. Lindahl T and Nyberg B. Rate of depurination of native deoxyribonucleic acid. Biochemistry 11: 3610-3618, 1972.

307. Liu CS, Tsai CS, Kuo CL, Chen HW, Lii CK, Ma YS, and Wei YH. Oxidative stress-related alteration of the copy number of mitochondrial DNA in human leukocytes. Free Radic Res 37: 1307-1317, 2003.

308. Liu GH, Barkho BZ, Ruiz S, Diep D, Qu J, Yang SL, Panopoulos AD, Suzuki K, Kurian L, Walsh C, Thompson J, Boue S, Fung HL, Sancho-Martinez I, Zhang K, Yates J, 3rd, and Izpisua Belmonte JC. Recapitulation of premature ageing with iPSCs from Hutchinson-Gilford progeria syndrome. Nature 472: 221-225, 2011.

309. Liu X, Yamada N, Maruyama W, and Osawa T. Formation of dopamine adducts derived from brain polyunsaturated fatty acids: mechanism for Parkinson disease. $J$ Biol Chem 283: 34887-34895, 2008.

310. Liu Z, Sun Y, Tan S, Liu L, Hu S, Huo H, Li M, Cui Q, and $\mathrm{Yu}$ M. Nutrient deprivation-related OXPHOS/glycolysis interconversion via HIF-1alpha/C-MYC pathway in U251 cells. Tumour Biol 37: 6661-6671, 2016.

311. Lombard J. Autism: a mitochondrial disorder? Med Hypotheses 50: 497-500, 1998.

312. Lopez-Gonzalez R, Lu Y, Gendron TF, Karydas A, Tran H, Yang D, Petrucelli L, Miller BL, Almeida S, and Gao FB. Poly(GR) in C9ORF72-related ALS/FTD compromises mitochondrial function and increases oxidative stress and DNA damage in iPSC-derived motor neurons. Neuron 92: 383-391, 2016.

313. Lotharius $\mathbf{J}$ and Brundin P. Pathogenesis of Parkinson's disease: dopamine, vesicles and alpha-synuclein. Nat Rev Neurosci 3: 932-942, 2002.

314. Lotharius J and O'Malley KL. The parkinsonism-inducing drug 1-methyl-4-phenylpyridinium triggers intracellular dopamine oxidation. A novel mechanism of toxicity. $J$ Biol Chem 275: 38581-38588, 2000. 
315. Lu T, Aron L, Zullo J, Pan Y, Kim H, Chen Y, Yang TH, Kim HM, Drake D, Liu XS, Bennett DA, Colaiacovo MP, and Yankner BA. REST and stress resistance in ageing and Alzheimer's disease. Nature 507: 448-454, 2014.

316. Lu XH, Mattis VB, Wang N, Al-Ramahi I, van den Berg N, Fratantoni SA, Waldvogel H, Greiner E, Osmand A, Elzein K, Xiao J, Dijkstra S, de Pril R, Vinters HV, Faull R, Signer E, Kwak S, Marugan JJ, Botas J, Fischer DF, Svendsen CN, Munoz-Sanjuan I, and Yang XW. Targeting ATM ameliorates mutant Huntingtin toxicity in cell and animal models of Huntington's disease. Sci Transl Med 6: 268ra178, 2014.

317. Lu YF, Kandel ER, and Hawkins RD. Nitric oxide signaling contributes to late-phase LTP and CREB phosphorylation in the hippocampus. J Neurosci 19: 10250 10261, 1999.

318. Luc R and Vergely C. Forgotten radicals in biology. Int $J$ BioMed Sci 4: 255-259, 2008.

319. Luo Y, Bolon B, Kahn S, Bennett BD, Babu-Khan S, Denis P, Fan W, Kha H, Zhang J, Gong Y, Martin L, Louis JC, Yan Q, Richards WG, Citron M, and Vassar R. Mice deficient in BACE1, the Alzheimer's beta-secretase, have normal phenotype and abolished beta-amyloid generation. Nat Neurosci 4: 231-232, 2001.

320. Luth ES, Stavrovskaya IG, Bartels T, Kristal BS, and Selkoe DJ. Soluble, prefibrillar alpha-synuclein oligomers promote complex I-dependent, $\mathrm{Ca}^{2+}$-induced mitochondrial dysfunction. J Biol Chem 289: 21490-21507, 2014.

321. Ma MW, Wang J, Zhang Q, Wang R, Dhandapani KM, Vadlamudi RK, and Brann DW. NADPH oxidase in brain injury and neurodegenerative disorders. Mol Neurodegener 12: 7, 2017.

322. Machamer CE. The Golgi complex in stress and death. Front Neurosci 9: 421, 2015.

323. Maiuri T, Mocle AJ, Hung CL, Xia J, van Roon-Mom WM, and Truant R. Huntingtin is a scaffolding protein in the ATM oxidative DNA damage response complex. Hum Mol Genet 26: 395-406, 2017.

324. Majounie E, Renton AE, Mok K, Dopper EG, Waite A, Rollinson S, Chio A, Restagno G, Nicolaou N, SimonSanchez J, van Swieten JC, Abramzon Y, Johnson JO, Sendtner M, Pamphlett R, Orrell RW, Mead S, Sidle KC, Houlden H, Rohrer JD, Morrison KE, Pall H, Talbot K, and Ansorge O; Chromosome 9-ALS/FTD Consortium; French Research Network on FTLD/FTLD/ALS; ITALSGEN Consortium; Hernandez DG, Arepalli S, Sabatelli M, Mora G, Corbo M, Giannini F, Calvo A, Englund E, Borghero G, Floris GL, Remes AM, Laaksovirta H, McCluskey L, Trojanowski JQ, Van Deerlin VM, Schellenberg GD, Nalls MA, Drory VE, Lu CS, Yeh TH, Ishiura H, Takahashi Y, Tsuji S, Le Ber I, Brice A, Drepper C, Williams N, Kirby J, Shaw P, Hardy J, Tienari PJ, Heutink P, Morris HR, Pickering-Brown S, and Traynor BJ. Frequency of the C9orf72 hexanucleotide repeat expansion in patients with amyotrophic lateral sclerosis and frontotemporal dementia: a cross-sectional study. Lancet Neurol 11: 323-330, 2012.

325. Mandavilli BS, Santos JH, and Van Houten B. Mitochondrial DNA repair and aging. Mutat Res 509: 127151, 2002.

326. Manta B and Gladyshev VN. Regulated methionine oxidation by monooxygenases. Free Radic Biol Med 109: 141-155, 2017.

327. Marangi G and Traynor BJ. Genetic causes of amyotrophic lateral sclerosis: new genetic analysis methodol- ogies entailing new opportunities and challenges. Brain Res 1607: 75-93, 2015.

328. Markey SP, Johannessen JN, Chiueh CC, Burns RS, and Herkenham MA. Intraneuronal generation of a pyridinium metabolite may cause drug-induced parkinsonism. Nature 311: 464-467, 1984.

329. Marklund SL. Human copper-containing superoxide dismutase of high molecular weight. Proc Natl Acad Sci U S A 79: 7634-7638, 1982.

330. Marklund SL. Extracellular superoxide dismutase and other superoxide dismutase isoenzymes in tissues from nine mammalian species. Biochem J 222: 649-655, 1984.

331. Marklund SL, Holme E, and Hellner L. Superoxide dismutase in extracellular fluids. Clin Chim Acta 126: 41-51, 1982.

332. Marmolino D, Manto M, Acquaviva F, Vergara P, Ravella A, Monticelli A, and Pandolfo M. PGC-1alpha downregulation affects the antioxidant response in Friedreich's ataxia. PLoS One 5: e10025, 2010.

333. Martin-Bastida A, Lao-Kaim NP, Loane C, Politis M, Roussakis AA, Valle-Guzman N, Kefalopoulou Z, PaulVisse G, Widner H, Xing Y, Schwarz ST, Auer DP, Foltynie T, Barker RA, and Piccini P. Motor associations of iron accumulation in deep grey matter nuclei in Parkinson's disease: a cross-sectional study of iron-related magnetic resonance imaging susceptibility. Eur J Neurol 24: 357-365, 2017.

334. Martinez TN and Greenamyre JT. Toxin models of mitochondrial dysfunction in Parkinson's disease. Antioxid Redox Signal 16: 920-934, 2012.

335. Masoud ST, Vecchio LM, Bergeron Y, Hossain MM, Nguyen LT, Bermejo MK, Kile B, Sotnikova TD, Siesser WB, Gainetdinov RR, Wightman RM, Caron MG, Richardson JR, Miller GW, Ramsey AJ, Cyr M, and Salahpour A. Increased expression of the dopamine transporter leads to loss of dopamine neurons, oxidative stress and L-dopa reversible motor deficits. Neurobiol Dis 74: 66-75, 2015.

336. Masters CL, Bateman R, Blennow K, Rowe CC, Sperling RA, and Cummings JL. Alzheimer's disease. Nat Rev Dis Primers 1: 15056, 2015.

337. Matsuda Y, Higashiyama S, Kijima Y, Suzuki K, Kawano K, Akiyama M, Kawata S, Tarui S, Deutsch HF, and Taniguchi N. Human liver manganese superoxide dismutase. Purification and crystallization, subunit association and sulfhydryl reactivity. Eur J Biochem 194: 713720, 1990.

338. Mattiazzi M, D’Aurelio M, Gajewski CD, Martushova K, Kiaei M, Beal MF, and Manfredi G. Mutated human SOD1 causes dysfunction of oxidative phosphorylation in mitochondria of transgenic mice. J Biol Chem 277: 29626-29633, 2002.

339. Mattson MP. Challenging oneself intermittently to improve health. Dose Response 12: 600-618, 2014.

340. McCord JM. The evolution of free radicals and oxidative stress. Am J Med 108: 652-659, 2000.

341. McCord JM and Fridovich I. Superoxide dismutase. An enzymic function for erythrocuprein (hemocuprein). $J$ Biol Chem 244: 6049-6055, 1969.

342. McGeer PL, Itagaki S, Akiyama H, and McGeer EG. Rate of cell death in parkinsonism indicates active neuropathological process. Ann Neurol 24: 574-576, 1988.

343. McKinnon PJ. ATM and the molecular pathogenesis of ataxia telangiectasia. Апnи Rev Pathol 7: 303-321, 2012. 
344. Mecocci P, MacGarvey U, Kaufman AE, Koontz D, Shoffner JM, Wallace DC, and Beal MF. Oxidative damage to mitochondrial DNA shows marked agedependent increases in human brain. Ann Neurol 34: 609616, 1993.

345. Menzies FM, Cookson MR, Taylor RW, Turnbull DM, Chrzanowska-Lightowlers ZM, Dong L, Figlewicz DA, and Shaw PJ. Mitochondrial dysfunction in a cell culture model of familial amyotrophic lateral sclerosis. Brain 125: 1522-1533, 2002.

346. Michalek M, Salnikov ES, and Bechinger B. Structure and topology of the huntingtin 1-17 membrane anchor by a combined solution and solid-state NMR approach. Biophys $J$ 105: 699-710, 2013.

347. Michihara A, Shimatani M, Anraku M, Tomida H, and Akasaki K. High levels of oxidative stress exist in the brain than serum or kidneys in stroke-prone spontaneously hypertensive rats at ten weeks of age. Biol Pharm Bull 33: 518-521, 2010.

348. Mishanina TV, Libiad M, and Banerjee R. Biogenesis of reactive sulfur species for signaling by hydrogen sulfide oxidation pathways. Nat Chem Biol 11: 457-464, 2015.

349. Mollereau B, Rzechorzek NM, Roussel BD, Sedru M, Van den Brink DM, Bailly-Maitre B, Palladino F, Medinas DB, Domingos PM, Hunot S, Chandran S, Birman S, Baron T, Vivien D, Duarte CB, Ryoo HD, Steller H, Urano F, Chevet E, Kroemer G, Ciechanover A, Calabrese EJ, Kaufman RJ, and Hetz C. Adaptive preconditioning in neurological diseases - therapeutic insights from proteostatic perturbations. Brain Res 1648: 603-616, 2016.

350. Molokanova E, Akhtar MW, Sanz-Blasco S, Tu S, PinaCrespo JC, McKercher SR, and Lipton SA. Differential effects of synaptic and extrasynaptic NMDA receptors on Abeta-induced nitric oxide production in cerebrocortical neurons. J Neurosci 34: 5023-5028, 2014.

351. Molteni $\mathrm{M}$ and Rossetti C. Neurodegenerative diseases: the immunological perspective. J Neuroimmunol 313: 109-115, 2017.

352. Montarolo F, Perga S, Martire S, Navone DN, Marchet A, Leotta D, and Bertolotto A. Altered NR4A subfamily gene expression level in peripheral blood of Parkinson's and Alzheimer's disease patients. Neurotox Res 30: 338-344, 2016.

353. Morgan B, Ezerina D, Amoako TN, Riemer J, Seedorf M, and Dick TP. Multiple glutathione disulfide removal pathways mediate cytosolic redox homeostasis. Nat Chem Biol 9: 119-125, 2013.

354. Moriarty-Craige SE, Adkison J, Lynn M, Gensler G, Bressler S, Jones DP, and Sternberg P, Jr. Antioxidant supplements prevent oxidation of cysteine/cystine redox in patients with age-related macular degeneration. Am J Ophthalmol 140: 1020-1026, 2005.

355. Mosharov EV, Larsen KE, Kanter E, Phillips KA, Wilson K, Schmitz Y, Krantz DE, Kobayashi K, Edwards RH, and Sulzer D. Interplay between cytosolic dopamine, calcium, and alpha-synuclein causes selective death of substantia nigra neurons. Neuron 62: 218-229, 2009.

356. Moskovitz J. Methionine sulfoxide reductases: ubiquitous enzymes involved in antioxidant defense, protein regulation, and prevention of aging-associated diseases. Biochim Biophys Acta 1703: 213-219, 2005.

357. Moyse E, Arseneault M, Gaudreau P, Ferland G, and Ramassamy C. Gender- and region-dependent changes of redox biomarkers in the brain of successfully aging LOU/ C rats. Mech Ageing Dev 149: 19-30, 2015.

358. Murphy MP. How mitochondria produce reactive oxygen species. Biochem J 417: 1-13, 2009.

359. Mustacich DJ, Bruno RS, and Traber MG. Vitamin E. Vitam Horm 76: 1-21, 2007.

360. Mustafa AK, Gadalla MM, Sen N, Kim S, Mu W, Gazi SK, Barrow RK, Yang G, Wang R, and Snyder SH. $\mathrm{H}_{2} \mathrm{~S}$ signals through protein S-sulfhydration. Sci Signal 2: ra72, 2009.

361. Nagano S, Takahashi Y, Yamamoto K, Masutani H, Fujiwara N, Urushitani M, and Araki T. A cysteine residue affects the conformational state and neuronal toxicity of mutant SOD1 in mice: relevance to the pathogenesis of ALS. Hum Mol Genet 24: 3427-3439, 2015.

362. Nakamura $\mathrm{T}$ and Lipton SA. Redox modulation by Snitrosylation contributes to protein misfolding, mitochondrial dynamics, and neuronal synaptic damage in neurodegenerative diseases. Cell Death Differ 18: 14781486, 2011.

363. Nakamura T and Lipton SA. Protein S-nitrosylation as a therapeutic target for neurodegenerative diseases. Trends Pharmacol Sci 37: 73-84, 2016.

364. Narendra D, Tanaka A, Suen DF, and Youle RJ. Parkin is recruited selectively to impaired mitochondria and promotes their autophagy. J Cell Biol 183: 795-803, 2008.

365. Nayernia Z, Jaquet V, and Krause KH. New insights on NOX enzymes in the central nervous system. Antioxid Redox Signal 20: 2815-2837, 2014.

366. Nguyen AD, Itoh S, Jeney V, Yanagisawa H, Fujimoto M, Ushio-Fukai M, and Fukai T. Fibulin-5 is a novel binding protein for extracellular superoxide dismutase. Circ Res 95: 1067-1074, 2004.

367. Nguyen VD, Saaranen MJ, Karala AR, Lappi AK, Wang L, Raykhel IB, Alanen HI, Salo KE, Wang CC, and Ruddock LW. Two endoplasmic reticulum PDI peroxidases increase the efficiency of the use of peroxide during disulfide bond formation. J Mol Biol 406: 503-515, 2011.

368. Nishino T, Okamoto K, Eger BT, Pai EF, and Nishino T. Mammalian xanthine oxidoreductase-mechanism of transition from xanthine dehydrogenase to xanthine oxidase. FEBS J 275: 3278-3289, 2008.

369. Nobrega-Pereira S, Fernandez-Marcos PJ, Brioche T, Gomez-Cabrera MC, Salvador-Pascual A, Flores JM, Vina J, and Serrano M. G6PD protects from oxidative damage and improves healthspan in mice. Nat Commun 7: 10894, 2016.

370. O'Rourke JG, Bogdanik L, Muhammad A, Gendron TF, Kim KJ, Austin A, Cady J, Liu EY, Zarrow J, Grant S, Ho R, Bell S, Carmona S, Simpkinson M, Lall D, Wu K, Daughrity L, Dickson DW, Harms MB, Petrucelli L, Lee EB, Lutz CM, and Baloh RH. C9orf72 BAC transgenic mice display typical pathologic features of ALS/FTD. Neuron 88: 892-901, 2015.

371. Ogilby PR. Singlet oxygen: there is still something new under the sun, and it is better than ever. Photochem Photobiol Sci 9: 1543-1560, 2010.

372. Ohno K, Okuda K, and Uehara T. Endogenous Ssulfhydration of PTEN helps protect against modification by nitric oxide. Biochem Biophys Res Commun 456: 245249, 2015.

373. Oleinick NL, Morris RL, and Belichenko I. The role of apoptosis in response to photodynamic therapy: what, 
where, why, and how. Photochem Photobiol Sci 1: 1-21, 2002.

374. Olson GE, Whitin JC, Hill KE, Winfrey VP, Motley AK, Austin LM, Deal J, Cohen HJ, and Burk RF. Extracellular glutathione peroxidase (Gpx3) binds specifically to basement membranes of mouse renal cortex tubule cells. Am J Physiol Renal Physiol 298: F1244-F1253, 2010.

375. Orr HT, Chung MY, Banfi S, Kwiatkowski TJ, Jr., Servadio $\mathrm{A}$, Beaudet $\mathrm{AL}$, McCall $\mathrm{AE}$, Duvick LA, Ranum LP, and Zoghbi HY. Expansion of an unstable trinucleotide CAG repeat in spinocerebellar ataxia type 1. Nat Genet 4: 221-226, 1993.

376. Orrell RW, Lane RJ, and Ross M. A systematic review of antioxidant treatment for amyotrophic lateral sclerosis/ motor neuron disease. Amyotroph Lateral Scler 9: 195211, 2008.

377. Pal R, Bajaj L, Sharma J, Palmieri M, Di Ronza A, Lotfi P, Chaudhury A, Neilson J, Sardiello M, and Rodney GG. NADPH oxidase promotes Parkinsonian phenotypes by impairing autophagic flux in an mTORC1-independent fashion in a cellular model of Parkinson's disease. Sci Rep 6: 22866, 2016.

378. Papa S. Mitochondrial oxidative phosphorylation changes in the life span. Molecular aspects and physiopathological implications. Biochim Biophys Acta 1276: 87-105, 1996.

379. Parker WD, Jr., Parks JK, and Swerdlow RH. Complex I deficiency in Parkinson's disease frontal cortex. Brain Res 1189: 215-218, 2008.

380. Pasca SP, Nemes B, Vlase L, Gagyi CE, Dronca E, Miu AC, and Dronca M. High levels of homocysteine and low serum Paraoxonase 1 arylesterase activity in children with autism. Life Sci 78: 2244-2248, 2006.

381. Paul BD, Sbodio JI, Xu R, Vandiver MS, Cha JY, Snowman AM, and Snyder SH. Cystathionine gammalyase deficiency mediates neurodegeneration in Huntington's disease. Nature 509: 96-100, 2014.

382. Paul BD and Snyder $\mathrm{SH} . \mathrm{H}_{2} \mathrm{~S}$ signalling through protein sulfhydration and beyond. Nat Rev Mol Cell Biol 13: 499_ 507, 2012.

383. Paul BD and Snyder SH. Neurodegeneration in Huntington's disease involves loss of cystathionine gamma-lyase. Cell Cycle 13: 2491-2493, 2014.

384. Paul BD and Snyder SH. $\mathrm{H}_{2} \mathrm{~S}$ : a novel gasotransmitter that signals by sulfhydration. Trends Biochem Sci 40: 687700, 2015.

385. Paul BD and Snyder SH. Modes of physiologic $\mathrm{H}_{2} \mathrm{~S}$ signaling in the brain and peripheral tissues. Antioxid Redox Signal 22: 411-423, 2015.

386. Paul BD and Snyder SH. Protein sulfhydration. Methods Enzymol 555: 79-90, 2015.

387. Pearce RK, Owen A, Daniel S, Jenner P, and Marsden CD. Alterations in the distribution of glutathione in the substantia nigra in Parkinson's disease. J Neural Transm (Vienna) 104: 661-677, 1997.

388. Perez RG, Waymire JC, Lin E, Liu JJ, Guo F, and Zigmond MJ. A role for alpha-synuclein in the regulation of dopamine biosynthesis. J Neurosci 22: 3090-3099, 2002.

389. Perez VI, Bokov A, Van Remmen H, Mele J, Ran Q, Ikeno Y, and Richardson A. Is the oxidative stress theory of aging dead? Biochim Biophys Acta 1790: 1005-1014, 2009.

390. Perez VI, Buffenstein R, Masamsetti V, Leonard S, Salmon AB, Mele J, Andziak B, Yang T, Edrey Y, Friguet B, Ward W, Richardson A, and Chaudhuri A. Protein sta- bility and resistance to oxidative stress are determinants of longevity in the longest-living rodent, the naked mole-rat. Proc Natl Acad Sci U S A 106: 3059-3064, 2009.

391. Perry TL, Godin DV, and Hansen S. Parkinson's disease: a disorder due to nigral glutathione deficiency? Neurosci Lett 33: 305-310, 1982.

392. Perry TL and Yong VW. Idiopathic Parkinson's disease, progressive supranuclear palsy and glutathione metabolism in the substantia nigra of patients. Neurosci Lett 67: 269-274, 1986.

393. Persico AM and Napolioni V. Autism genetics. Behav Brain Res 251: 95-112, 2013.

394. Peters OM, Cabrera GT, Tran H, Gendron TF, McKeon JE, Metterville J, Weiss A, Wightman N, Salameh J, Kim J, Sun H, Boylan KB, Dickson D, Kennedy Z, Lin Z, Zhang YJ, Daughrity L, Jung C, Gao FB, Sapp PC, Horvitz HR, Bosco DA, Brown SP, de Jong P, Petrucelli L, Mueller C, and Brown RH, Jr. Human C9ORF72 hexanucleotide expansion reproduces RNA foci and dipeptide repeat proteins but not neurodegeneration in BAC transgenic mice. Neuron 88: 902-909, 2015.

395. Petersen SV, Oury TD, Ostergaard L, Valnickova Z, Wegrzyn J, Thogersen IB, Jacobsen C, Bowler RP, Fattman CL, Crapo JD, and Enghild JJ. Extracellular superoxide dismutase (EC-SOD) binds to type I collagen and protects against oxidative fragmentation. $J$ Biol Chem 279: 13705-13710, 2004.

396. Pham-Huy LA, He H, and Pham-Huy C. Free radicals, antioxidants in disease and health. Int J BioMed Sci 4: 8996, 2008.

397. Pickrell AM and Youle RJ. The roles of PINK1, parkin, and mitochondrial fidelity in Parkinson's disease. Neuron 85: 257-273, 2015.

398. Pitts MW, Byrns CN, Ogawa-Wong AN, Kremer P, and Berry MJ. Selenoproteins in nervous system development and function. Biol Trace Elem Res 161: 231-245, 2014.

399. Poewe W, Seppi K, Tanner CM, Halliday GM, Brundin P, Volkmann J, Schrag AE, and Lang AE. Parkinson disease. Nat Rev Dis Prim 3: 17013, 2017.

400. Polymeropoulos MH, Higgins JJ, Golbe LI, Johnson WG, Ide SE, Di Iorio G, Sanges G, Stenroos ES, Pho LT, Schaffer AA, Lazzarini AM, Nussbaum RL, and Duvoisin RC. Mapping of a gene for Parkinson's disease to chromosome 4q21-q23. Science 274: 1197-1199, 1996.

401. Polymeropoulos MH, Lavedan C, Leroy E, Ide SE, Dehejia A, Dutra A, Pike B, Root H, Rubenstein J, Boyer R, Stenroos ES, Chandrasekharappa S, Athanassiadou A, Papapetropoulos T, Johnson WG, Lazzarini AM, Duvoisin RC, Di Iorio G, Golbe LI, and Nussbaum RL. Mutation in the alpha-synuclein gene identified in families with Parkinson's disease. Science 276: 2045-2047, 1997.

402. Poole LB. The basics of thiols and cysteines in redox biology and chemistry. Free Radic Biol Med 80: 148-157, 2015.

403. Przedborski S, Jackson-Lewis V, Yokoyama R, Shibata T, Dawson VL, and Dawson TM. Role of neuronal nitric oxide in 1-methyl-4-phenyl-1,2,3,6-tetrahydropyridine (MPTP)-induced dopaminergic neurotoxicity. Proc Natl Acad Sci U S A 93: 4565-4571, 1996.

404. Puccio H, Simon D, Cossee M, Criqui-Filipe P, Tiziano F, Melki J, Hindelang C, Matyas R, Rustin P, and Koenig M. Mouse models for Friedreich ataxia exhibit cardiomyopathy, sensory nerve defect and Fe-S enzyme deficiency followed by intramitochondrial iron deposits. Nat Genet 27: 181-186, 2001. 
405. Purdue PE and Lazarow PB. Targeting of human catalase to peroxisomes is dependent upon a novel $\mathrm{COOH}$-terminal peroxisomal targeting sequence. J Cell Biol 134: 849-862, 1996.

406. Putnam CD, Arvai AS, Bourne Y, and Tainer JA. Active and inhibited human catalase structures: ligand and NADPH binding and catalytic mechanism. J Mol Biol 296: 295-309, 2000.

407. Quijano C, Hernandez-Saavedra D, Castro L, McCord JM, Freeman BA, and Radi R. Reaction of peroxynitrite with Mn-superoxide dismutase. Role of the metal center in decomposition kinetics and nitration. J Biol Chem 276: 11631-11638, 2001.

408. Radi R. Peroxynitrite, a stealthy biological oxidant. J Biol Chem 288: 26464-26472, 2013.

409. Radi R, Beckman JS, Bush KM, and Freeman BA. Peroxynitrite oxidation of sulfhydryls. The cytotoxic potential of superoxide and nitric oxide. J Biol Chem 266: 4244-4250, 1991.

410. Raefsky SM and Mattson MP. Adaptive responses of neuronal mitochondria to bioenergetic challenges: roles in neuroplasticity and disease resistance. Free Radic Biol Med 102: 203-216, 2017.

411. Raha S and Robinson BH. Mitochondria, oxygen free radicals, disease and ageing. Trends Biochem Sci 25: 502508, 2000

412. Rajendran R, Minqin R, Ynsa MD, Casadesus G, Smith MA, Perry G, Halliwell B, and Watt F. A novel approach to the identification and quantitative elemental analysis of amyloid deposits-insights into the pathology of Alzheimer's disease. Biochem Biophys Res Commun 382: 91-95, 2009.

413. Ramsay RR, Dadgar J, Trevor A, and Singer TP. Energydriven uptake of N-methyl-4-phenylpyridine by brain mitochondria mediates the neurotoxicity of MPTP. Life Sci 39: 581-588, 1986.

414. Ramsay RR, Kowal AT, Johnson MK, Salach JI, and Singer TP. The inhibition site of $\mathrm{MPP}^{+}$, The neurotoxic bioactivation product of 1-methyl-4-phenyl-1,2,3,6tetrahydropyridine is near the Q-binding site of NADH dehydrogenase. Arch Biochem Biophys 259: 645-649, 1987.

415. Ramsay RR, Salach JI, and Singer TP. Uptake of the neurotoxin 1-methyl-4-phenylpyridine $\left(\mathrm{MPP}^{+}\right)$by mitochondria and its relation to the inhibition of the mitochondrial oxidation of $\mathrm{NAD}^{+}$-linked substrates by $\mathrm{MPP}^{+}$. Biochem Biophys Res Commun 134: 743-748, 1986.

416. Ranguelova $\mathrm{K}$, Rice $\mathrm{AB}$, Lardinois OM, Triquigneaux M, Steinckwich N, Deterding LJ, Garantziotis S, and Mason RP. Sulfite-mediated oxidation of myeloperoxidase to a free radical: immuno-spin trapping detection in human neutrophils. Free Radic Biol Med 60: 98-106, 2013.

417. Ravindranath V, Shivakumar BR, and Anandatheerthavarada HK. Low glutathione levels in brain regions of aged rats. Neurosci Lett 101: 187-190, 1989.

418. Reaume AG, Elliott JL, Hoffman EK, Kowall NW, Ferrante RJ, Siwek DF, Wilcox HM, Flood DG, Beal MF, Brown RH, Jr., Scott RW, and Snider WD. Motor neurons in $\mathrm{Cu} / \mathrm{Zn}$ superoxide dismutase-deficient mice develop normally but exhibit enhanced cell death after axonal injury. Nat Genet 13: 43-47, 1996.

419. Reed GA. Co-oxidation of xenobiotics: lipid peroxyl derivatives as mediators of metabolism. Chem Phys Lipids 44: 127-148, 1987.
420. Reliene R and Schiestl RH. Antioxidant N-acetyl cysteine reduces incidence and multiplicity of lymphoma in Atm deficient mice. DNA Repair (Amst) 5: 852-859, 2006.

421. Renton AE, Majounie E, Waite A, Simon-Sanchez J, Rollinson S, Gibbs JR, Schymick JC, Laaksovirta H, van Swieten JC, Myllykangas L, Kalimo H, Paetau A, Abramzon Y, Remes AM, Kaganovich A, Scholz SW, Duckworth J, Ding J, Harmer DW, Hernandez DG, Johnson JO, Mok K, Ryten M, Trabzuni D, Guerreiro RJ, Orrell RW, Neal J, Murray A, Pearson J, Jansen IE, Sondervan D, Seelaar H, Blake D, Young K, Halliwell N, Callister JB, Toulson G, Richardson A, Gerhard A, Snowden J, Mann D, Neary D, Nalls MA, Peuralinna T, Jansson L, Isoviita VM, Kaivorinne AL, Holtta-Vuori M, Ikonen E, Sulkava R, Benatar M, Wuu J, Chio A, Restagno G, Borghero G, Sabatelli M, Consortium I, Heckerman D, Rogaeva E, Zinman L, Rothstein JD, Sendtner M, Drepper C, Eichler EE, Alkan C, Abdullaev Z, Pack SD, Dutra A, Pak E, Hardy J, Singleton A, Williams NM, Heutink P, Pickering-Brown S, Morris HR, Tienari PJ, Traynor BJ, and Traynor BJ. A hexanucleotide repeat expansion in C9ORF72 is the cause of chromosome 9p21linked ALS-FTD. Neuron 72: 257-268, 2011.

422. Rhee SG, Kang SW, Chang TS, Jeong W, and Kim K. Peroxiredoxin, a novel family of peroxidases. IUBMB Life 52: 35-41, 2001.

423. Rhee SG, Woo HA, and Kang D. The role of peroxiredoxins in the transduction of $\mathrm{H}_{2} \mathrm{O}_{2}$ signals. Antioxid Redox Signal 28: 537-557, 2018.

424. Richter C, Park JW, and Ames BN. Normal oxidative damage to mitochondrial and nuclear DNA is extensive. Proc Natl Acad Sci U S A 85: 6465-6467, 1988.

425. Rivera-Torres J, Acin-Perez R, Cabezas-Sanchez P, Osorio FG, Gonzalez-Gomez C, Megias D, Camara C, Lopez-Otin C, Enriquez JA, Luque-Garcia JL, and Andres V. Identification of mitochondrial dysfunction in Hutchinson-Gilford progeria syndrome through use of stable isotope labeling with amino acids in cell culture. $J$ Proteomics 91: 466-477, 2013.

426. Roberds SL, Anderson J, Basi G, Bienkowski MJ, Branstetter DG, Chen KS, Freedman SB, Frigon NL, Games D, Hu K, Johnson-Wood K, Kappenman KE, Kawabe TT, Kola I, Kuehn R, Lee M, Liu W, Motter R, Nichols NF, Power M, Robertson DW, Schenk D, Schoor M, Shopp GM, Shuck ME, Sinha S, Svensson KA, Tatsuno G, Tintrup H, Wijsman J, Wright S, and McConlogue L. BACE knockout mice are healthy despite lacking the primary beta-secretase activity in brain: implications for Alzheimer's disease therapeutics. Hum Mol Genet 10: 1317-1324, 2001.

427. Robin ED and Wong R. Mitochondrial DNA molecules and virtual number of mitochondria per cell in mammalian cells. J Cell Physiol 136: 507-513, 1988.

428. Rockabrand E, Slepko N, Pantalone A, Nukala VN, Kazantsev A, Marsh JL, Sullivan PG, Steffan JS, Sensi SL, and Thompson LM. The first 17 amino acids of huntingtin modulate its sub-cellular localization, aggregation and effects on calcium homeostasis. Hum Mol Genet 16: 6177, 2007.

429. Rodriguez-Oroz MC, Jahanshahi M, Krack P, Litvan I, Macias R, Bezard E, and Obeso JA. Initial clinical manifestations of Parkinson's disease: features and pathophysiological mechanisms. Lancet Neurol 8: 1128-1139, 2009. 
430. Rodriguez-Rocha H, Garcia-Garcia A, Pickett C, Li S, Jones J, Chen H, Webb B, Choi J, Zhou Y, Zimmerman MC, and Franco R. Compartmentalized oxidative stress in dopaminergic cell death induced by pesticides and complex I inhibitors: distinct roles of superoxide anion and superoxide dismutases. Free Radic Biol Med 61: 370-383, 2013.

431. Rose S, Bennuri SC, Wynne R, Melnyk S, James SJ, and Frye RE. Mitochondrial and redox abnormalities in autism lymphoblastoid cells: a sibling control study. FASEB J 31: 904-909, 2017.

432. Rose S, Frye RE, Slattery J, Wynne R, Tippett M, Melnyk $\mathrm{S}$, and James SJ. Oxidative stress induces mitochondrial dysfunction in a subset of autistic lymphoblastoid cell lines. Transl Psychiatry 4: e377, 2014.

433. Rosen DR, Siddique T, Patterson D, Figlewicz DA, Sapp P, Hentati A, Donaldson D, Goto J, O'Regan JP, Deng HX, Rahmani Z, Krizus A, McKenna-Yasek D, Cayabyab A, Gaston SM, Berger R, Tanzi RE, Halperin JJ, Herzfeldt B, Van den Bergh R, Hung WY, Bird T, Deng G, Mulder DW, Smyth C, Laing NG, Soriano E, Pericak-Vance MA, Haines J, Rouleau GA, Gusella JS, Horvitz HR, and Brown Jr RH. Mutations in $\mathrm{Cu} / \mathrm{Zn}$ superoxide dismutase gene are associated with familial amyotrophic lateral sclerosis. Nature 362: 59-62, 1993.

434. Ross EK, Winter AN, Wilkins HM, Sumner WA, Duval N, Patterson D, and Linseman DA. A cystine-rich whey supplement (Immunocal((R))) delays disease onset and prevents spinal cord glutathione depletion in the hSOD1(G93A) mouse model of amyotrophic lateral sclerosis. Antioxidants (Basel) 3: 843-865, 2014.

435. Rotblat B, Southwell AL, Ehrnhoefer DE, Skotte NH, Metzler M, Franciosi S, Leprivier G, Somasekharan SP, Barokas A, Deng Y, Tang T, Mathers J, Cetinbas N, Daugaard M, Kwok B, Li L, Carnie CJ, Fink D, Nitsch R, Galpin JD, Ahern CA, Melino G, Penninger JM, Hayden $\mathrm{MR}$, and Sorensen PH. HACE1 reduces oxidative stress and mutant huntingtin toxicity by promoting the NRF2 response. Proc Natl Acad Sci U S A 111: 3032-3037, 2014.

436. Rothstein JD. Edaravone: a new drug approved for ALS. Cell 171: 725, 2017.

437. Rotig A, de Lonlay P, Chretien D, Foury F, Koenig M, Sidi D, Munnich A, and Rustin P. Aconitase and mitochondrial iron-sulphur protein deficiency in Friedreich ataxia. Nat Genet 17: 215-217, 1997.

438. Rouault TA. Iron metabolism in the CNS: implications for neurodegenerative diseases. Nat Rev Neurosci 14: 551564, 2013.

439. Rowland LP and Shneider NA. Amyotrophic lateral sclerosis. N Engl J Med 344: 1688-1700, 2001.

440. Rushmore TH, King RG, Paulson KE, and Pickett CB. Regulation of glutathione S-transferase Ya subunit gene expression: identification of a unique xenobioticresponsive element controlling inducible expression by planar aromatic compounds. Proc Natl Acad Sci U S A 87: 3826-3830, 1990.

441. Sahara N, Maeda S, Murayama M, Suzuki T, Dohmae N, Yen SH, and Takashima A. Assembly of two distinct dimers and higher-order oligomers from full-length tau. Eur J Neurosci 25: 3020-3029, 2007.

442. Saijo K, Winner B, Carson CT, Collier JG, Boyer L, Rosenfeld MG, Gage FH, and Glass CK. A Nurr1/CoREST pathway in microglia and astrocytes protects dopaminergic neurons from inflammation-induced death. Cell 137: 47-59, 2009.
443. Saito T, Shinzawa H, Togashi H, Wakabayashi H, Ukai K, Takahashi T, Ishikawa M, Dobashi M, and Imai Y. Ultrastructural localization of $\mathrm{Cu}, \mathrm{Zn}-\mathrm{SOD}$ in hepatocytes of patients with various liver diseases. Histol Histopathol 4: $1-6,1989$.

444. Salazar J, Mena N, Hunot S, Prigent A, Alvarez-Fischer D, Arredondo M, Duyckaerts C, Sazdovitch V, Zhao L, Garrick LM, Nunez MT, Garrick MD, Raisman-Vozari R, and Hirsch EC. Divalent metal transporter 1 (DMT1) contributes to neurodegeneration in animal models of Parkinson's disease. Proc Natl Acad Sci U S A 105: 18578-18583, 2008.

445. Samiec PS, Drews-Botsch C, Flagg EW, Kurtz JC, Sternberg P, Jr., Reed RL, and Jones DP. Glutathione in human plasma: decline in association with aging, agerelated macular degeneration, and diabetes. Free Radic Biol Med 24: 699-704, 1998.

446. Sandstrom J, Carlsson L, Marklund SL, and Edlund T. The heparin-binding domain of extracellular superoxide dismutase $\mathrm{C}$ and formation of variants with reduced heparin affinity. J Biol Chem 267: 18205-18209, 1992.

447. Sarsour EH, Kalen AL, and Goswami PC. Manganese superoxide dismutase regulates a redox cycle within the cell cycle. Antioxid Redox Signal 20: 1618-1627, 2014.

448. Sarsour EH, Kalen AL, Xiao Z, Veenstra TD, Chaudhuri L, Venkataraman S, Reigan P, Buettner GR, and Goswami PC. Manganese superoxide dismutase regulates a metabolic switch during the mammalian cell cycle. Cancer Res 72: 3807-3816, 2012.

449. Savitsky K, Bar-Shira A, Gilad S, Rotman G, Ziv Y, Vanagaite L, Tagle DA, Smith S, Uziel T, Sfez S, Ashkenazi M, Pecker I, Frydman M, Harnik R, Patanjali SR, Simmons A, Clines GA, Sartiel A, Gatti RA, Chessa L, Sanal O, Lavin MF, Jaspers NG, Taylor AM, Arlett CF, Miki T, Weissman SM, Lovett M, Collins FS, and Shiloh Y. A single ataxia telangiectasia gene with a product similar to PI-3 kinase. Science 268: 1749-1753, 1995.

450. Savitsky K, Sfez S, Tagle DA, Ziv Y, Sartiel A, Collins FS, Shiloh Y, and Rotman G. The complete sequence of the coding region of the ATM gene reveals similarity to cell cycle regulators in different species. Hum Mol Genet 4: 2025-2032, 1995.

451. Saxena $S$ and Caroni P. Selective neuronal vulnerability in neurodegenerative diseases: from stressor thresholds to degeneration. Neuron 71: 35-48, 2011.

452. Sbodio JI, Paul BD, Machamer CE, and Snyder SH. Golgi protein ACBD3 mediates neurotoxicity associated with Huntington's disease. Cell Rep 4: 890-897, 2013.

453. Sbodio JI, Snyder SH, and Paul BD. Transcriptional control of amino acid homeostasis is disrupted in Huntington's disease. Proc Natl Acad Sci U S A 113: 88438848, 2016.

454. Sbodio JI, Snyder SH, and Paul BD. Golgi stress response reprograms cysteine metabolism to confer cytoprotection in Huntington's disease. Proc Natl Acad Sci U S A 115: 780-785, 2018.

455. Schapira AH, Cooper JM, Dexter D, Jenner P, Clark JB, and Marsden CD. Mitochondrial complex I deficiency in Parkinson's disease. Lancet 1: 1269, 1989.

456. Schneider M, Forster H, Boersma A, Seiler A, Wehnes H, Sinowatz F, Neumuller C, Deutsch MJ, Walch A, Hrabe de Angelis M, Wurst W, Ursini F, Roveri A, Maleszewski M, Maiorino M, and Conrad M. Mitochondrial glutathione 
peroxidase 4 disruption causes male infertility. FASEB $J$ 23: 3233-3242, 2009.

457. Schoneich C. Sulfur radical-induced redox modifications in proteins: analysis and mechanistic aspects. Antioxid Redox Signal 26: 388-405, 2017.

458. Schrag A and Schott JM. Epidemiological, clinical, and genetic characteristics of early-onset parkinsonism. Lancet Neurol 5: 355-363, 2006.

459. Schriner SE, Linford NJ, Martin GM, Treuting P, Ogburn CE, Emond M, Coskun PE, Ladiges W, Wolf N, Van Remmen H, Wallace DC, and Rabinovitch PS. Extension of murine life span by overexpression of catalase targeted to mitochondria. Science 308: 1909-1911, 2005.

460. Schubert R, Erker L, Barlow C, Yakushiji H, Larson D, Russo A, Mitchell JB, and Wynshaw-Boris A. Cancer chemoprevention by the antioxidant tempol in Atmdeficient mice. Hum Mol Genet 13: 1793-1802, 2004.

461. Schulz JB, Matthews RT, Muqit MM, Browne SE, and Beal MF. Inhibition of neuronal nitric oxide synthase by 7-nitroindazole protects against MPTP-induced neurotoxicity in mice. J Neurochem 64: 936-939, 1995.

462. Searcy DG, Whitehead JP, and Maroney MJ. Interaction of $\mathrm{Cu}, \mathrm{Zn}$ superoxide dismutase with hydrogen sulfide. Arch Biochem Biophys 318: 251-263, 1995.

463. Sedlak TW, Saleh M, Higginson DS, Paul BD, Juluri KR, and Snyder SH. Bilirubin and glutathione have complementary antioxidant and cytoprotective roles. Proc Natl Acad Sci U S A 106: 5171-5176, 2009.

464. Sellier C, Campanari ML, Julie Corbier C, Gaucherot A, Kolb-Cheynel I, Oulad-Abdelghani M, Ruffenach F, Page A, Ciura S, Kabashi E, and Charlet-Berguerand N. Loss of C9ORF72 impairs autophagy and synergizes with polyQ ataxin-2 to induce motor neuron dysfunction and cell death. EMBO J 35: 1276-1297, 2016.

465. Sen N, Hara MR, Kornberg MD, Cascio MB, Bae BI, Shahani N, Thomas B, Dawson TM, Dawson VL, Snyder $\mathrm{SH}$, and Sawa A. Nitric oxide-induced nuclear GAPDH activates p300/CBP and mediates apoptosis. Nat Cell Biol 10: 866-873, 2008.

466. Sen N, Paul BD, Gadalla MM, Mustafa AK, Sen T, Xu R, Kim S, and Snyder SH. Hydrogen sulfide-linked sulfhydration of NF- $\kappa \mathrm{B}$ mediates its antiapoptotic actions. Mol Cell 45: 13-24, 2012.

467. Seong IS, Ivanova E, Lee JM, Choo YS, Fossale E, Anderson M, Gusella JF, Laramie JM, Myers RH, Lesort M, and MacDonald ME. HD CAG repeat implicates a dominant property of huntingtin in mitochondrial energy metabolism. Hum Mol Genet 14: 2871-2880, 2005.

468. Shay JW, Pierce DJ, and Werbin H. Mitochondrial DNA copy number is proportional to total cell DNA under a variety of growth conditions. J Biol Chem 265: 1480214807, 1990.

469. Sheng Y, Abreu IA, Cabelli DE, Maroney MJ, Miller AF, Teixeira M, and Valentine JS. Superoxide dismutases and superoxide reductases. Chem Rev 114: 3854-3918, 2014.

470. Shibuya N, Tanaka M, Yoshida M, Ogasawara Y, Togawa $\mathrm{T}$, Ishii K, and Kimura H. 3-Mercaptopyruvate sulfurtransferase produces hydrogen sulfide and bound sulfane sulfur in the brain. Antioxid Redox Signal 11: 703-714, 2009.

471. Sian-Hulsmann J, Mandel S, Youdim MB, and Riederer P. The relevance of iron in the pathogenesis of Parkinson's disease. J Neurochem 118: 939-957, 2011.
472. Sies H. Hydrogen peroxide as a central redox signaling molecule in physiological oxidative stress: oxidative eustress. Redox Biol 11: 613-619, 2017.

473. Sies H, Berndt C, and Jones DP. Oxidative stress. Annu Rev Biochem 86: 715-748, 2017.

474. Skibinski G, Hwang V, Ando DM, Daub A, Lee AK, Ravisankar A, Modan S, Finucane MM, Shaby BA, and Finkbeiner S. Nrf2 mitigates LRRK2- and alphasynuclein-induced neurodegeneration by modulating proteostasis. Proc Natl Acad Sci U S A 114: 1165-1170, 2017.

475. Sohal RS and Weindruch R. Oxidative stress, caloric restriction, and aging. Science 273: 59-63, 1996.

476. Sorce S, Stocker R, Seredenina T, Holmdahl R, Aguzzi A, Chio A, Depaulis A, Heitz F, Olofsson P, Olsson T, Duveau V, Sanoudou D, Skosgater S, Vlahou A, Wasquel D, Krause $\mathrm{KH}$, and Jaquet V. NADPH oxidases as drug targets and biomarkers in neurodegenerative diseases: what is the evidence? Free Radic Biol Med 112: 387-396, 2017.

477. Spector R and Lorenzo AV. Ascorbic acid homeostasis in the central nervous system. Am J Physiol 225: 757-763, 1973.

478. Spickett CM. The lipid peroxidation product 4-hydroxy-2nonenal: advances in chemistry and analysis. Redox Biol 1: 145-152, 2013

479. Spillantini MG, Schmidt ML, Lee VM, Trojanowski JQ, Jakes R, and Goedert M. Alpha-synuclein in Lewy bodies. Nature 388: 839-840, 1997.

480. Spiteller G and Afzal M. The action of peroxyl radicals, powerful deleterious reagents, explains why neither cholesterol nor saturated fatty acids cause atherogenesis and age-related diseases. Chemistry 20: 14928-14945, 2014.

481. Stack EC, Matson WR, and Ferrante RJ. Evidence of oxidant damage in Huntington's disease: translational strategies using antioxidants. Ann N Y Acad Sci 1147: 79-92, 2008.

482. Stadtman ER. Protein modification in aging. J Gerontol 43: B112-B120, 1988.

483. Stadtman ER, Moskovitz J, and Levine RL. Oxidation of methionine residues of proteins: biological consequences. Antioxid Redox Signal 5: 577-582, 2003.

484. Stamler JS, Simon DI, Osborne JA, Mullins ME, Jaraki O, Michel T, Singel DJ, and Loscalzo J. S-nitrosylation of proteins with nitric oxide: synthesis and characterization of biologically active compounds. Proc Natl Acad Sci U S A 89: 444-448, 1992.

485. Steffan JS, Agrawal N, Pallos J, Rockabrand E, Trotman LC, Slepko N, Illes K, Lukacsovich T, Zhu YZ, Cattaneo E, Pandolfi PP, Thompson LM, and Marsh JL. SUMO modification of huntingtin and Huntington's disease pathology. Science 304: 100-104, 2004.

486. Stirpe F and Della Corte E. The regulation of rat liver xanthine oxidase. Conversion in vitro of the enzyme activity from dehydrogenase (type D) to oxidase (type O). $J$ Biol Chem 244: 3855-3863, 1969.

487. Stocker R, Yamamoto Y, McDonagh AF, Glazer AN, and Ames BN. Bilirubin is an antioxidant of possible physiological importance. Science 235: 1043-1046, 1987.

488. Stocker S, Maurer M, Ruppert T, and Dick TP. A role for 2-Cys peroxiredoxins in facilitating cytosolic protein thiol oxidation. Nat Chem Biol 14: 148-155, 2018.

489. Stocker S, Van Laer K, Mijuskovic A, and Dick TP. The conundrum of hydrogen peroxide signaling and the emerging role of peroxiredoxins as redox relay hubs. Antioxid Redox Signal 28: 558-573, 2018.

490. Su B, Wang X, Lee HG, Tabaton M, Perry G, Smith MA, and Zhu X. Chronic oxidative stress causes increased tau 
phosphorylation in M17 neuroblastoma cells. Neurosci Lett 468: 267-271, 2010.

491. Sultana R and Butterfield DA. Role of oxidative stress in the progression of Alzheimer's disease. J Alzheimers Dis 19: 341-353, 2010.

492. Sulzer D, Bogulavsky J, Larsen KE, Behr G, Karatekin E, Kleinman MH, Turro N, Krantz D, Edwards RH, Greene LA, and Zecca L. Neuromelanin biosynthesis is driven by excess cytosolic catecholamines not accumulated by synaptic vesicles. Proc Natl Acad Sci U S A 97: 1186911874, 2000.

493. Sulzer D and Zecca L. Intraneuronal dopamine-quinone synthesis: a review. Neurotox Res 1: 181-195, 2000.

494. Sunico CR, Sultan A, Nakamura T, Dolatabadi N, Parker J, Shan B, Han X, Yates JR, 3rd, Masliah E, Ambasudhan $\mathrm{R}$, Nakanishi N, and Lipton SA. Role of sulfiredoxin as a peroxiredoxin-2 denitrosylase in human iPSC-derived dopaminergic neurons. Proc Natl Acad Sci U S A 113: E7564-E7571, 2016.

495. Surmeier DJ and Schumacker PT. Calcium, bioenergetics, and neuronal vulnerability in Parkinson's disease. J Biol Chem 288: 10736-10741, 2013.

496. Surmeier DJ, Schumacker PT, Guzman JD, Ilijic E, Yang B, and Zampese E. Calcium and Parkinson's disease. Biochem Biophys Res Commun 483: 1013-1019, 2017.

497. Suzuki T, Motohashi H, and Yamamoto M. Toward clinical application of the Keap1-Nrf2 pathway. Trends Pharmacol Sci 34: 340-346, 2013.

498. Tackenberg C, Grinschgl S, Trutzel A, Santuccione AC, Frey MC, Konietzko U, Grimm J, Brandt R, and Nitsch RM. NMDA receptor subunit composition determines beta-amyloid-induced neurodegeneration and synaptic loss. Cell Death Dis 4: e608, 2013.

499. Tainer JA, Getzoff ED, Beem KM, Richardson JS, and Richardson DC. Determination and analysis of the 2 Astructure of copper, zinc superoxide dismutase. J Mol Biol 160: 181-217, 1982.

500. Takahashi H, Xia P, Cui J, Talantova M, Bodhinathan K, Li W, Saleem S, Holland EA, Tong G, Pina-Crespo J, Zhang D, Nakanishi N, Larrick JW, McKercher SR, Nakamura T, Wang Y, and Lipton SA. Pharmacologically targeted NMDA receptor antagonism by nitromemantine for cerebrovascular disease. Sci Rep 5: 14781, 2015.

501. Talantova M, Sanz-Blasco S, Zhang X, Xia P, Akhtar MW, Okamoto S, Dziewczapolski G, Nakamura T, Cao G, Pratt AE, Kang YJ, Tu S, Molokanova E, McKercher SR, Hires SA, Sason H, Stouffer DG, Buczynski MW, Solomon JP, Michael S, Powers ET, Kelly JW, Roberts A, Tong G, Fang-Newmeyer T, Parker J, Holland EA, Zhang D, Nakanishi N, Chen HS, Wolosker H, Wang Y, Parsons LH, Ambasudhan R, Masliah E, Heinemann SF, Pina-Crespo JC, and Lipton SA. Abeta induces astrocytic glutamate release, extrasynaptic NMDA receptor activation, and synaptic loss. Proc Natl Acad Sci U S A 110: E2518-E2527, 2013.

502. Tanaka S, Uehara T, and Nomura Y. Up-regulation of protein-disulfide isomerase in response to hypoxia/brain ischemia and its protective effect against apoptotic cell death. J Biol Chem 275: 10388-10393, 2000.

503. Tang G, Gutierrez Rios P, Kuo SH, Akman HO, Rosoklija G, Tanji K, Dwork A, Schon EA, Dimauro S, Goldman J, and Sulzer D. Mitochondrial abnormalities in temporal lobe of autistic brain. Neurobiol Dis 54: 349-361, 2013.
504. Tao R, Coleman MC, Pennington JD, Ozden O, Park SH, Jiang H, Kim HS, Flynn CR, Hill S, Hayes McDonald W, Olivier AK, Spitz DR, and Gius D. Sirt3-mediated deacetylation of evolutionarily conserved lysine 122 regulates MnSOD activity in response to stress. Mol Cell 40: 893904, 2010.

505. Tapias V, Hu X, Luk KC, Sanders LH, Lee VM, and Greenamyre JT. Synthetic alpha-synuclein fibrils cause mitochondrial impairment and selective dopamine neurodegeneration in part via iNOS-mediated nitric oxide production. Cell Mol Life Sci 74: 2851-2874, 2017.

506. Tarrago L and Gladyshev VN. Recharging oxidative protein repair: catalysis by methionine sulfoxide reductases towards their amino acid, protein, and model substrates. Biochem Mosc 77: 1097-1107, 2012.

507. Taylor JP, Brown RH, Jr., and Cleveland DW. Decoding ALS: from genes to mechanism. Nature 539: 197-206, 2016.

508. Thompson LM, Aiken CT, Kaltenbach LS, Agrawal N, Illes K, Khoshnan A, Martinez-Vincente M, Arrasate M, O'Rourke JG, Khashwji H, Lukacsovich T, Zhu YZ, Lau AL, Massey A, Hayden MR, Zeitlin SO, Finkbeiner S, Green KN, LaFerla FM, Bates G, Huang L, Patterson PH, Lo DC, Cuervo AM, Marsh JL, and Steffan JS. IKK phosphorylates huntingtin and targets it for degradation by the proteasome and lysosome. J Cell Biol 187: 10831099, 2009.

509. Tibell L, Hjalmarsson K, Edlund T, Skogman G, Engstrom A, and Marklund SL. Expression of human extracellular superoxide dismutase in Chinese hamster ovary cells and characterization of the product. Proc Natl Acad Sci U S A 84: 6634-6638, 1987.

510. Tiong CX, Lu M, and Bian JS. Protective effect of hydrogen sulphide against 6-OHDA-induced cell injury in SH-SY5Y cells involves PKC/PI3K/Akt pathway. $\mathrm{Br} \mathrm{J}$ Pharmacol 161: 467-480, 2010.

511. Twelves D, Perkins KS, and Counsell C. Systematic review of incidence studies of Parkinson's disease. Mov Disord 18: 19-31, 2003.

512. Uehara T, Nakamura $T$, Yao D, Shi ZQ, Gu Z, Ma Y, Masliah E, Nomura Y, and Lipton SA. S-nitrosylated protein-disulphide isomerase links protein misfolding to neurodegeneration. Nature 441: 513-517, 2006.

513. Uthus EO and Brown-Borg HM. Methionine flux to transsulfuration is enhanced in the long living Ames dwarf mouse. Mech Ageing Dev 127: 444-450, 2006.

514. Uttara B, Singh AV, Zamboni P, and Mahajan RT. Oxidative stress and neurodegenerative diseases: a review of upstream and downstream antioxidant therapeutic options. Curr Neuropharmacol 7: 65-74, 2009.

515. Uversky VN, Li J, and Fink AL. Metal-triggered structural transformations, aggregation, and fibrillation of human alpha-synuclein. A possible molecular NK between Parkinson's disease and heavy metal exposure. J Biol Chem 276: 44284-44296, 2001.

516. Valencia A, Sapp E, Kimm JS, McClory H, Reeves PB, Alexander J, Ansong KA, Masso N, Frosch MP, Kegel $\mathrm{KB}$, Li X, and DiFiglia M. Elevated NADPH oxidase activity contributes to oxidative stress and cell death in Huntington's disease. Hum Mol Genet 22: 1112-1131, 2013.

517. van Duijn E. Medical treatment of behavioral manifestations of Huntington disease. Handb Clin Neurol 144: 129139, 2017. 
518. Van Houten B, Woshner V, and Santos JH. Role of mitochondrial DNA in toxic responses to oxidative stress. DNA Repair (Amst) 5: 145-152, 2006.

519. Vandiver MS, Paul BD, Xu R, Karuppagounder S, Rao F, Snowman AM, Ko HS, Lee YI, Dawson VL, Dawson TM, Sen N, and Snyder SH. Sulfhydration mediates neuroprotective actions of parkin. Nat Commun 4: 1626, 2013.

520. Vargas MR, Johnson DA, and Johnson JA. Decreased glutathione accelerates neurological deficit and mitochondrial pathology in familial ALS-linked hSOD1(G93A) mice model. Neurobiol Dis 43: 543-551, 2011.

521. Vassar R, Bennett BD, Babu-Khan S, Kahn S, Mendiaz EA, Denis P, Teplow DB, Ross S, Amarante P, Loeloff R, Luo Y, Fisher S, Fuller J, Edenson S, Lile J, Jarosinski MA, Biere AL, Curran E, Burgess T, Louis JC, Collins F, Treanor J, Rogers G, and Citron M. Beta-secretase cleavage of Alzheimer's amyloid precursor protein by the transmembrane aspartic protease BACE. Science 286: 735-741, 1999.

522. Vekrellis K, Xilouri M, Emmanouilidou E, Rideout HJ, and Stefanis L. Pathological roles of alpha-synuclein in neurological disorders. Lancet Neurol 10: 1015-1025, 2011.

523. Vural G, Gumusyayla S, Bektas H, Deniz O, Alisik M, and Erel O. Impairment of dynamic thiol-disulphide homeostasis in patients with idiopathic Parkinson's disease and its relationship with clinical stage of disease. Clin Neurol Neurosurg 153: 50-55, 2017.

524. Wallace DC. Mitochondrial genetics: a paradigm for aging and degenerative diseases? Science 256: 628-632, 1992.

525. Wang JQ, Chen Q, Wang X, Wang QC, Wang Y, Cheng HP, Guo C, Sun Q, Chen Q, and Tang TS. Dysregulation of mitochondrial calcium signaling and superoxide flashes cause mitochondrial genomic DNA damage in Huntington disease. J Biol Chem 288: 3070-3084, 2013.

526. Wang Q, Li WX, Dai SX, Guo YC, Han FF, Zheng JJ, Li $\mathrm{GH}$, and Huang JF. Meta-analysis of Parkinson's disease and Alzheimer's disease revealed commonly impaired pathways and dysregulation of NRF2-dependent genes. $J$ Alzheimers Dis 56: 1525-1539, 2017.

527. Wang QM, Xu YY, Liu S, and Ma ZG. Isradipine attenuates MPTP-induced dopamine neuron degeneration by inhibiting up-regulation of L-type calcium channels and iron accumulation in the substantia nigra of mice. Oncotarget 8: 47284-47295, 2017.

528. Wang W, Nguyen LT, Burlak C, Chegini F, Guo F, Chataway T, Ju S, Fisher OS, Miller DW, Datta D, Wu F, Wu CX, Landeru A, Wells JA, Cookson MR, Boxer MB, Thomas CJ, Gai WP, Ringe D, Petsko GA, and Hoang QQ. Caspase-1 causes truncation and aggregation of the Parkinson's disease-associated protein alpha-synuclein. Proc Natl Acad Sci U S A 113: 9587-9592, 2016.

529. Wang $X$ and Michaelis EK. Selective neuronal vulnerability to oxidative stress in the brain. Front Aging Neurosci 2: 12, 2010.

530. Webster CP, Smith EF, Bauer CS, Moller A, Hautbergue GM, Ferraiuolo L, Myszczynska MA, Higginbottom A, Walsh MJ, Whitworth AJ, Kaspar BK, Meyer K, Shaw PJ, Grierson AJ, and De Vos KJ. The C9orf72 protein interacts with Rab1a and the ULK1 complex to regulate initiation of autophagy. EMBO J 35: 1656-1676, 2016.

531. Weiduschat N, Mao X, Hupf J, Armstrong N, Kang G, Lange DJ, Mitsumoto H, and Shungu DC. Motor cortex glutathione deficit in ALS measured in vivo with the $\mathrm{J}$ editing technique. Neurosci Lett 570: 102-107, 2014.

532. Weisiger RA and Fridovich I. Mitochondrial superoxide simutase. Site of synthesis and intramitochondrial localization. J Biol Chem 248: 4793-4796, 1973.

533. Weyemi U, Redon CE, Aziz T, Choudhuri R, Maeda D, Parekh PR, Bonner MY, Arbiser JL, and Bonner WM. NADPH oxidase 4 is a critical mediator in ataxia telangiectasia disease. Proc Natl Acad Sci U S A 112: 21212126, 2015.

534. Whitehead RE, Ferrer JV, Javitch JA, and Justice JB. Reaction of oxidized dopamine with endogenous cysteine residues in the human dopamine transporter. J Neurochem 76: 1242-1251, 2001.

535. Wilson RB and Roof DM. Respiratory deficiency due to loss of mitochondrial DNA in yeast lacking the frataxin homologue. Nat Genet 16: 352-357, 1997.

536. Winner B, Jappelli R, Maji SK, Desplats PA, Boyer L, Aigner S, Hetzer C, Loher T, Vilar M, Campioni S, Tzitzilonis C, Soragni A, Jessberger S, Mira H, Consiglio A, Pham E, Masliah E, Gage FH, and Riek R. In vivo demonstration that alpha-synuclein oligomers are toxic. Proc Natl Acad Sci U S A 108: 4194-4199, 2011.

537. Winterbourn CC. Reconciling the chemistry and biology of reactive oxygen species. Nat Chem Biol 4: 278-286, 2008.

538. Winterbourn CC. The biological chemistry of hydrogen peroxide. Methods Enzymol 528: 3-25, 2013.

539. Winterbourn CC, Kettle AJ, and Hampton MB. Reactive oxygen species and neutrophil function. Annu Rev Biochem 85: 765-792, 2016.

540. Wispe JR, Clark JC, Burhans MS, Kropp KE, Korfhagen TR, and Whitsett JA. Synthesis and processing of the precursor for human mangano-superoxide dismutase. Biochim Biophys Acta 994: 30-36, 1989.

541. Woo HA, Kang SW, Kim HK, Yang KS, Chae HZ, and Rhee SG. Reversible oxidation of the active site cysteine of peroxiredoxins to cysteine sulfinic acid. Immunoblot detection with antibodies specific for the hyperoxidized cysteine-containing sequence. J Biol Chem 278: 4736147364, 2003.

542. Wright DJ, Renoir T, Smith ZM, Frazier AE, Francis PS, Thorburn DR, McGee SL, Hannan AJ, and Gray LJ. Nacetylcysteine improves mitochondrial function and ameliorates behavioral deficits in the R6/1 mouse model of Huntington's disease. Transl Psychiatry 5: e492, 2015.

543. Writing G; Edaravone (MCI-186) ALS 19 Study Group. Safety and efficacy of edaravone in well defined patients with amyotrophic lateral sclerosis: a randomised, doubleblind, placebo-controlled trial. Lancet Neurol 16: 505$512,2017$.

544. Wu DC, Teismann P, Tieu K, Vila M, Jackson-Lewis V, Ischiropoulos $\mathrm{H}$, and Przedborski S. NADPH oxidase mediates oxidative stress in the 1-methyl-4-phenyl1,2,3,6-tetrahydropyridine model of Parkinson's disease. Proc Natl Acad Sci U S A 100: 6145-6150, 2003.

545. Xia P, Chen HS, Zhang D, and Lipton SA. Memantine preferentially blocks extrasynaptic over synaptic NMDA receptor currents in hippocampal autapses. J Neurosci 30: 11246-11250, 2010.

546. Xiao G, Fan Q, Wang X, and Zhou B. Huntington disease arises from a combinatory toxicity of polyglutamine and copper binding. Proc Natl Acad Sci U S A 110: 1499515000, 2013. 
547. Xie L, Hu LF, Teo XQ, Tiong CX, Tazzari V, Sparatore A, Del Soldato P, Dawe GS, and Bian JS. Therapeutic effect of hydrogen sulfide-releasing L-Dopa derivative ACS84 on 6-OHDA-induced Parkinson's disease rat model. PLoS One 8: e60200, 2013.

548. Yamazaki I and Piette LH. ESR spin-trapping studies on the reaction of $\mathrm{Fe}^{2+}$ ions with $\mathrm{H}_{2} \mathrm{O}_{2}$-reactive species in oxygen toxicity in biology. J Biol Chem 265: 13589 13594, 1990.

549. Yang G, Zhao K, Ju Y, Mani S, Cao Q, Puukila S, Khaper $\mathrm{N}$, Wu L, and Wang R. Hydrogen sulfide protects against cellular senescence via S-sulfhydration of Keap1 and activation of Nrf2. Antioxid Redox Signal 18: 1906-1919, 2013.

550. Yang WS and Stockwell BR. Ferroptosis: death by lipid peroxidation. Trends Cell Biol 26: 165-176, 2016.

551. Yant LJ, Ran Q, Rao L, Van Remmen H, Shibatani T, Belter JG, Motta L, Richardson A, and Prolla TA. The selenoprotein GPX4 is essential for mouse development and protects from radiation and oxidative damage insults. Free Radic Biol Med 34: 496-502, 2003.

552. Yao M, Nguyen TV, and Pike CJ. Beta-amyloid-induced neuronal apoptosis involves c-Jun $\mathrm{N}$-terminal kinasedependent downregulation of Bcl-w. J Neurosci 25: 11491158, 2005.

553. Yarza R, Vela S, Solas M, and Ramirez MJ. c-Jun Nterminal kinase (JNK) signaling as a therapeutic target for Alzheimer's disease. Front Pharmacol 6: 321, 2015.

554. Yen TC, Su JH, King KL, and Wei YH. Ageingassociated $5 \mathrm{~kb}$ deletion in human liver mitochondrial DNA. Biochem Biophys Res Commun 178: 124-131, 1991.

555. Yin $\mathrm{H}, \mathrm{Xu} \mathrm{L}$, and Porter NA. Free radical lipid peroxidation: mechanisms and analysis. Chem Rev 111: 5944 5972, 2011.

556. Yoh K, Itoh K, Enomoto A, Hirayama A, Yamaguchi N, Kobayashi M, Morito N, Koyama A, Yamamoto M, and Takahashi S. Nrf2-deficient female mice develop lupuslike autoimmune nephritis. Kidney Int 60: 1343-1353, 2001.

557. Yoshida T, Maulik N, Engelman RM, Ho YS, and Das DK. Targeted disruption of the mouse Sod I gene makes the hearts vulnerable to ischemic reperfusion injury. Circ Res 86: 264-269, 2000.

558. Yoshida T, Maulik N, Engelman RM, Ho YS, Magnenat JL, Rousou JA, Flack JE, 3rd, Deaton D, and Das DK. Glutathione peroxidase knockout mice are susceptible to myocardial ischemia reperfusion injury. Circulation 96: II-216-II-220, 1997.

559. You Z, Cao X, Taylor AB, Hart PJ, and Levine RL. Characterization of a covalent polysulfane bridge in copper-zinc superoxide dismutase. Biochemistry 49: 1191-1198, 2010.

560. Zaichick SV, McGrath KM, and Caraveo G. The role of $\mathrm{Ca}^{2+}$ signaling in Parkinson's disease. Dis Model Mech 10: 519-535, 2017.

561. Zhang C, Yang L, Wang XB, Wang JS, Geng YD, Yang $\mathrm{CS}$, and Kong LY. Calyxin Y induces hydrogen peroxidedependent autophagy and apoptosis via JNK activation in human non-small cell lung cancer NCI-H460 cells. Cancer Lett 340: 51-62, 2013.

562. Zhang DD, Lo SC, Cross JV, Templeton DJ, and Hannink M. Keap1 is a redox-regulated substrate adaptor protein for a Cul3-dependent ubiquitin ligase complex. Mol Cell Biol 24: 10941-10953, 2004.

563. Zhang S, Rocourt C, and Cheng WH. Selenoproteins and the aging brain. Mech Ageing Dev 131: 253-260, 2010.

564. Zhang W, Wang T, Pei Z, Miller DS, Wu X, Block ML, Wilson B, Zhang W, Zhou Y, Hong JS, and Zhang J. Aggregated alpha-synuclein activates microglia: a process leading to disease progression in Parkinson's disease. FASEB J 19: 533-542, 2005.

565. Zhang $\mathrm{X}, \mathrm{Yu} \mathrm{L}$, and $\mathrm{Xu} \mathrm{H}$. Lysosome calcium in ROS regulation of autophagy. Autophagy 12: 1954-1955, 2016.

566. Zhang Y, Hodgson NW, Trivedi MS, Abdolmaleky HM, Fournier M, Cuenod M, Do KQ, and Deth RC. Decreased brain levels of vitamin B12 in aging, autism and schizophrenia. PLoS One 11: e0146797, 2016.

567. Zhang Y, Zhou Y, Schweizer U, Savaskan NE, Hua D, Kipnis J, Hatfield DL, and Gladyshev VN. Comparative analysis of selenocysteine machinery and selenoproteome gene expression in mouse brain identifies neurons as key functional sites of selenium in mammals. $J$ Biol Chem 283: 2427-2438, 2008.

568. Zhang Z, Liu L, Jiang X, Zhai S, and Xing D. The essential role of Drp1 and its regulation by S-Nitrosylation of parkin in dopaminergic neurodegeneration: implications for Parkinson's disease. Antioxid Redox Signal 25: 609-622, 2016.

569. Zhu X, Lee HG, Casadesus G, Avila J, Drew K, Perry G, and Smith MA. Oxidative imbalance in Alzheimer's disease. Mol Neurobiol 31: 205-217, 2005.

570. Zhu X, Raina AK, Rottkamp CA, Aliev G, Perry G, Boux $\mathrm{H}$, and Smith MA. Activation and redistribution of c-jun $\mathrm{N}$-terminal kinase/stress activated protein kinase in degenerating neurons in Alzheimer's disease. J Neurochem 76: 435-441, 2001.

571. Ziering A and Lee J. Piperidine derivatives; 1,3-dialkyl-4aryl-4-acyloxypiperidines. J Org Chem 12: 911-914, 1947.

572. Zucca FA, Basso E, Cupaioli FA, Ferrari E, Sulzer D, Casella L, and Zecca L. Neuromelanin of the human substantia nigra: an update. Neurotox Res 25: 13-23, 2014.

573. Zuccato C, Ciammola A, Rigamonti D, Leavitt BR, Goffredo D, Conti L, MacDonald ME, Friedlander RM, Silani V, Hayden MR, Timmusk T, Sipione S, and Cattaneo E. Loss of huntingtin-mediated BDNF gene transcription in Huntington's disease. Science 293: 493-498, 2001.

Address correspondence to: Dr. Solomon H. Snyder

The Solomon H. Snyder Department of Neuroscience The Johns Hopkins University School of Medicine Baltimore, MD 21205

E-mail: ssnyder@jhmi.edu

Dr. Bindu D. Paul

The Solomon H. Snyder Department of Neuroscience The Johns Hopkins University School of Medicine Baltimore, MD 21205

E-mail: bpaul8@jhmi.edu

Date of first submission to ARS Central, August 10, 2017; date of final revised submission, March 16, 2018; date of acceptance, March 18, 2018. 

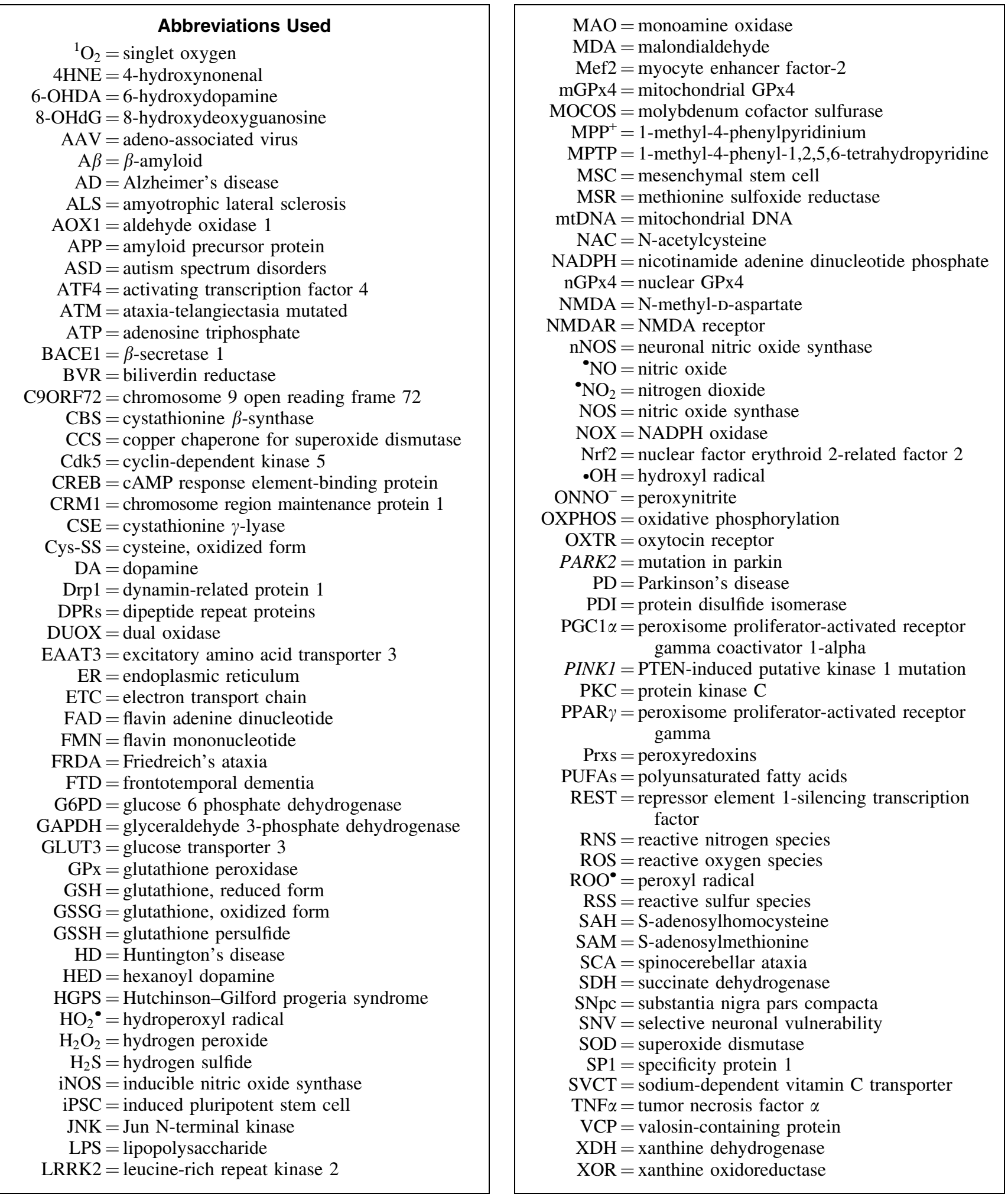